Editorial Manager(tm) for Graefe's Archive for Clinical and Experimental

Ophthalmology

Manuscript Draft

Manuscript Number: GRAEFES-D-10-00467R1

Title: Measuring Macular Pigment Optical Density In Vivo: A Review Of Techniques

Article Type: Review Article

Keywords: fundus autofluorescence; fundus reflectometry; heterochromatic flicker photometry; macular pigment; macular pigment optical density; motion photometry; Raman spectroscopy

Corresponding Author: Olivia Howells, BSc (Hons)

Corresponding Author's Institution:

First Author: Olivia Howells, BSc (Hons)

Order of Authors: Olivia Howells, BSc (Hons);Frank Eperjesi, PhD;Hannah Bartlett, PhD

Abstract: Background: Macular pigment has been the focus of much attention in recent years, as a potential modifiable risk factor for age-related macular degeneration. This interest has been

heightened by the ability to measure macular pigment optical density (MPOD) in vivo. Method: A systematic literature search was undertaken to identify all available papers that have used in vivo MPOD techniques. The papers were reviewed and all relevant information was incorporated into this article. Results: Measurement of MPOD is achievable with a wide range of techniques, which are typically categorized into one of two groups: psychophysical (requiring a response from the subject) or objective (requiring minimal input from the subject). The psychophysical methods include heterochromatic flicker photometry and minimum motion photometry. The objective methods include fundus reflectometry, fundus autofluorescence, resonance Raman spectroscopy and visual evoked potentials. Even within the individual techniques, there is often much variation in how data is obtained and processed. Conclusion: This review comprehensively details the procedure, instrumentation, assumptions, validity and reliability of each MPOD measurement technique currently available, along with their respective advantages and disadvantages. This leads us to conclude that development of a commercial instrument, based on fundus reflectometry or fundus autofluorescence, would be beneficial to macular pigment research and would support MPOD screening in a clinical setting. 


\section{${ }^{\star}$ Authorship Form}

Click here to download Authorship Form: Authorship Form.pdf

Authorship Form: Graefes Archive for Clinical and Experimental Ophthalmology

Title:-MEASURING MACULAR PIGMENT OPTICAL DENSITY IN VIVO: AREVIEW OF TECHINIQUES.

I, OLIVIA HOWELLS hereby confirm that all named authors meet the ICMJE

(corresponding author)

requirement of authorship and meet all three criteria as mentioned below:

1) substantial contributions to conception and design, or acquisition of data, or analysis and interpretation of data;

2) drafting the article or revising it critically for important intellectual content; and

3) final approval of the version to be published.

Authors should meet conditions 1, 2, and 3 .

*signed:

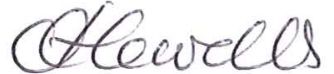

signed:

signed:

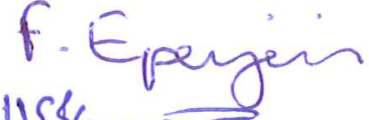

signed:

signed:

signed:

signed:

signed:

signed:

*First signature should be of corresponding author date: $01 / 06 / 2010$

date: $1 \mid 6 / 2010$

date: $1 / 6 / 2010$

date:

date:

date:

date:

date:

date:

The acknowledgment section includes contributors who provided purely technical help, writing assistance, or a department chair who provided only general support. Medical Writers; Financial and material support should also be acknowledged.

Groups of persons who have contributed materially to the paper but whose contributions do not justify authorship may be listed under a heading such as 'clinical investigators' or 'participating investigators,' and their function or contribution should be described-for example, 'served as scientific advisors,' 'critically reviewed the sludy proposal,' 'collected data,' or 'provided and cared for study patients.'

I confirm that this paper is not being submitted simultaneously elsewhere.

signed:

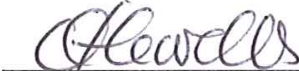
date: $01 / 06 / 2010$

(corresponding author) 


\section{Authors' Response to Reviewers' Comments}

Click here to download Authors' Response to Reviewers' Comments: File_1_Authors response to reviewers comments.docx

\section{Authors' response to reviewers' comments}

\section{$\underline{\text { Reviewer \#1 }}$}

It's a very well documented literature review about an interesting and up-to-date topic. Each technique reviewed is well described. It's easy to read, except some technical details wich could be difficult to understand for readers not used to take up this topic.

There are some changes to do.

1-First of all, the references in all the tables are wrong as none of them correspond to the authors listed. This must be corrected.

Thanks to the reviewer for spotting this error. This has been corrected in the submitted revision.

2-About autofluorescence technique, the problems encountered with lens opacities are not evoked. Cataract does not allow to obtain good images, and the macular pigment measurement is really affected in this case.

We have looked again at the research papers on the autofluorescence technique and can only find a very brief mention of the problems caused by lens opacities in a paper by Canovas et al. (2010). One of the repeatedly mentioned advantages of the autofluorescence technique over reflectometry in all the other papers is that it is little influenced by lens scatter and media opacities. We have, however, added the reviewer's point to the disadvantages in the autofluorescence section.

3-The AF technique should be better illustrated, showing for example the MP density profiles that are obtained (cf Wolf-Schnurrbusch UEK [153]).

We agree with the reviewer and have added the following text and figure 14 to the validity section:

'Figure 14, for instance, shows two distinct MPOD spatial profiles from a study by Wolf-Schnurrbusch et al. [154]. The top image is the classic distribution of a central peak in macular pigment with a rapid decline as distance from the fovea increases. The bottom image has a central peak in macular pigment followed by a decline, and there is also a secondary peak (a 'parafoveal ring') before further decline. In this particular study, the average eccentricity of the parafoveal ring was $0.66^{\circ}$ from the fovea, which is in line with several other studies [59, 116, 117]. Many researchers now propose that the total complement of macular pigment, rather than the peak amount, may better represent an individual's risk for, or protection from, AMD (see review by Bernstein et al. [155]).'

\section{Reviewer \#2:}

This is a good review article showing available methods of macular pigment optical density measurement. The following points are recommended/ need explanation from the author:

1-In some of the reference tags there is (e.g ?.) what do you mean by that? For example page 3 line 25.

We do use 'e.g.' a lot because there are so many papers that we could have used as references. It would be difficult to include them all and in our opinion it would make the paper cumbersome and difficult for the reader to penetrate. We would prefer to continue to use 'e.g.' in this style unless instructed otherwise by the Editor.

2-Page 7 line 27 (developed by 48 ) what do you mean by 48 ??

This has been clarified and now reads '(developed by Mellerio et al. [48]).' 


\section{3-Page 11 line 32 " for in vivo measurement of MPOD, validity is proved????." The sentence is repeated in the next paragraph under Validity and should be deleted.}

We are not sure what the reviewer means by this comment. Is the word 'proved' the problem here? Just in case it is we have replaced the word 'proved' with the word 'demonstrated'. We have checked again and the actual sentence is definitely not repeated in the next paragraph.

\section{4-Page 29 line 5 (see 161 for details) is that a reference??}

This has been clarified and now reads '(see Trieschmann et al. [162] for details)'

We also found another example of this: '(derived by 183)', from page 36, so this now reads '(derived by Hammond and Wooten [184])' - reference number altered due to update below.

\section{Note 1}

There has been a very recent article published since our paper was submitted. In order to make sure our review paper is as up to date as possible we have included information from this article. In the recent development section of HFP (page 8/9) we have included a minor up date from Nolan et al. (2010) (as well as Connolly et al. 2010 - already in review paper):

'Most recently, a further development on customized HFP and inverse yoking has been described by Connolly et al. [78] and Nolan et al. [97]. Whilst sticking with the traditional method of HFP MPOD determination, i.e. the radiance of blue light being increased/decreased until the point of minimal flicker, it takes on a more automated approach, as per the device of van der Veen et al. [53]. The instrument's electronics increase/decrease the amount of blue in the stimulus at a set rate. This removes any inter-individual variability in the speed at which the blue-green ratio is adjusted, therefore improving the accuracy of the MPOD value.'

All referencing has been updated accordingly.

Note 2

Some of our figure legends didn't explain exactly what technique was being used. Therefore we have updated these too (highlighted in revised manuscript). 


\title{
Measuring Macular Pigment Optical Density In Vivo: A Review Of Techniques
}

Olivia Howells, Frank Eperjesi, Hannah Bartlett

Ophthalmic Research Group, School of Life and Health Sciences, Aston University, Birmingham, B4 7ET, UK

Corresponding author: Olivia Howells; o.howells@aston.ac.uk; Tel. 0121204 4135;

Fax. 01212044048

\begin{abstract}
Background: Macular pigment has been the focus of much attention in recent years, as a potential modifiable risk factor for age-related macular degeneration. This interest has been heightened by the ability to measure macular pigment optical density (MPOD) in vivo. Method: A systematic literature search was undertaken to identify all available papers that have used in vivo MPOD techniques. The papers were reviewed and all relevant information was incorporated into this article. Results: Measurement of MPOD is achievable with a wide range of techniques, which are typically categorized into one of two groups: psychophysical (requiring a response from the subject) or objective (requiring minimal input from the subject). The psychophysical methods include heterochromatic flicker photometry and minimum motion photometry. The objective methods include fundus reflectometry, fundus autofluorescence, resonance Raman spectroscopy and visual evoked potentials. Even within the individual techniques, there is often much variation in how data is obtained and processed. Conclusion: This review comprehensively details the procedure, instrumentation, assumptions, validity and reliability of each MPOD measurement technique currently available, along with their respective advantages and disadvantages. This leads us to conclude that development of a commercial instrument, based on fundus reflectometry or fundus autofluorescence, would be beneficial to macular pigment research and would support MPOD screening in a clinical setting.
\end{abstract}

Keywords: fundus autofluorescence; fundus reflectometry; heterochromatic flicker photometry; macular pigment; macular pigment optical density; motion photometry; Raman spectroscopy. 


\section{INTRODUCTION}

Macular pigment is the collective name for three carotenoids, lutein, zeaxanthin and mesozeaxanthin, which are found at higher concentrations in the retina than anywhere else in the body, and to the exclusion of all other carotenoids [1]. They are only accessible to the body by dietary intake of foodstuffs or supplements containing them [2, 3], with high levels being found in certain fruits and vegetables, such as kiwi fruit, corn and spinach, as well as egg yolks [4].

Analysis of donor maculae is possibly the most unequivocal approach for assessing the distribution of macular pigment in the retina, and pioneering work by Snodderly and colleagues in the 1980 s achieved this $[5,6]$. Using primate monkeys and the technique of microdensitometry, it was confirmed, as expected, that macular pigment reaches its peak in the centre of the retina. There was then a sharp decline to negligible levels at approximately $1 \mathrm{~mm}\left(4^{\circ}\right)$ from the central fovea. In 1988, Bone et al., using high-performance liquid chromatography (HPLC), investigated the spatial distribution of macular pigment in human donors; in this case, it was found to reach negligible levels at $7^{\circ}$ eccentricity [7]. Within the retinal layers, macular pigment is primarily located in the photoreceptor axons and to a lesser extent in the inner plexiform layer [6, 8].

The macular carotenoids have an absorption spectrum of $400-540 \mathrm{~nm}$, peaking at approximately $460 \mathrm{~nm}$ [9]. This spectral peak, along with the spatial distribution and retinal layer localization of macular pigment contribute to its proposed function as a blue light filter. Short-wavelength (blue) light is more damaging to the retina than longer-wavelength light [10] so by attenuating the amount of blue light reaching the photoreceptors, macular pigment may protect the macula from this photo-damage; the higher the density of macular pigment (macular pigment optical density, or MPOD), the greater the amount of blue light filtering that will occur $[11,12]$. A second proposed function of macular pigment is that it protects the macula against oxidative stress by acting as an antioxidant [e.g. 13]. These blue light filter and antioxidant functions have led to the school of thought that having a high MPOD could help to protect against the eye disease age-related macular degeneration (AMD), the most prevalent cause of severe visual impairment in Western society [14-16]. As a result, there have been a multitude of studies investigating possible links between MPOD and AMD, using a variety of measurement techniques. Some of these studies have supported an MPOD-AMD association [e.g. 17, 18, 19] and some have not [e.g. 20, 21]. This inconsistent evidence is not too surprising, given the apparent multifactorial nature of AMD. What's more, 
it is highly likely that an individual's MPOD is equally multifactorial, but as one of the few potentially modifiable risk factors for AMD, its continued investigation is extremely important.

Macular pigment optical density may be measured in vitro or in vivo. In vitro measurement involves the techniques of HPLC [e.g. 22, 23] or microdensitometry [e.g. 24]. However, they can only be performed on excised retinas and so are clearly not suitable for widespread use. This review therefore details the most common techniques currently used to measure MPOD in vivo. These in vivo techniques are noninvasive and are normally categorized under one of two headings: psychophysical (requiring a response from the subject) or objective (requiring minimal input from the subject). Together they have established that MPOD varies widely between individuals, from virtually no macular pigment to greater than 1 log unit optical density, with average levels ranging from 0.16 [25] to 0.50 [26], depending on the method and/or the study population.

\section{METHOD}

A systematic literature search was conducted using ISI Web of Knowledge and PubMed. Key words and their combinations used for the search included 'macular pigment', 'macular pigment optical density', 'lutein', 'zeaxanthin', 'heterochromatic flicker photometry', 'motion photometry', reflectometry', 'autofluorescence', 'Raman', 'electrophysiology', and 'macular degeneration'. Further searches were undertaken for key researchers in the field such as Beatty, Berendschot, Bernstein, Bone, Delori, Gellermann, Hammond, Landrum, Moreland, Nolan, Robson, Snodderly, Stringham, Trieschmann, van de Kraats, and Wenzel. Further papers were obtained from the references of the retrieved articles. All the articles were reviewed and relevant information was incorporated into the manuscript.

\section{RESULTS OF REVIEW}

\section{Psychophysical techniques}

Psychophysical techniques of measuring macular pigment optical density (MPOD) include the following:

Threshold spectral sensitivity [e.g. 27, 28-31].

Colour matching [e.g. 32, 33-35].

Dichroism-based measurements [e.g. 9, 36].

Minimum motion photometry and apparent motion photometry [e.g. 37, 38-41]. 
Heterochromatic flicker photometry [e.g. 42, 43-53].

The first three of the psychophysical methods have now been largely superseded by heterochromatic flicker photometry (HFP) and, to some extent, minimum motion photometry. This is in part due to their increased level of difficulty and/or the longer time needed to perform them [54]. This review will therefore focus on the latter two methods. For information regarding the threshold sensitivity, colour matching and dichroism techniques, the reader is directed to the referenced studies, along with a validity review by Hammond et al. [54].

\section{Heterochromatic flicker photometry}

Developed by Ives in the early 1900s [55], HFP has so far been the most commonly used of all the techniques for measuring MPOD. As such, it is often used as a standard against which other techniques are validated [e.g. 56, 57-60], although at present there is no true 'gold-standard' in vivo measure of MPOD.

The use of HFP to measure macular pigment levels was first described over 30 years ago by Werner and Wooten [61] but the technique wasn't elaborated on until 1987, in a key paper by Werner, Donnelly and Kliegl [42]. Since then, HFP has been developed and used by numerous research groups investigating macular pigment. Key papers incorporating detailed descriptions and variations of the technique include Hammond and Fuld [43], Hammond et al. [44], Landrum et al. [45], Wooten et al. [46], Beatty et al. [47], Mellerio et al. [48], Bone and Landrum [49], Snodderly et al. [50], Tang et al. [62], lannaccone et al. [51], Stringham et al. [52], and van der Veen et al. [53]. All other studies using HFP to measure MPOD tend to use the instruments originally designed or developed by these investigators.

\section{Procedure}

In conjunction with many of the MPOD techniques, HFP exploits the spectral absorption properties and retinal location of macular pigment. Essentially, HFP determines MPOD by presenting a light stimulus of two alternating wavelengths at the fovea and at a parafoveal area. The wavelengths are chosen such that one is a short wavelength blue light that is maximally absorbed by macular pigment and the other is a longer wavelength green to yellow light that is not absorbed by macular pigment [6]. If the colours are alternated at an appropriate frequency and the luminance of the two colours is not perceived to be equal by the subject, the stimulus will appear as a flickering light; the perceived colour of this light will be an amalgamation of the two source colours [47, 49, 50,63]. Typically, the radiance (often 
also termed intensity) of the blue light is adjusted by the subject until the observed flicker is minimized [e.g. 44, 45, 46, 48, 50, 52, 62]. This occurs when there is an equiluminance match between the blue and green lights $[63,64]$. The procedure is then repeated at a parafoveal locus where macular pigment is negligible [65]. Since more blue light will be absorbed by macular pigment at the fovea than the parafovea, a greater radiance of blue light will be required at the fovea to appreciate minimal flicker. The log ratio of the radiance of blue light needed at the fovea compared with that needed at the parafovea gives a measure of peak MPOD (Formula 1), although whether this is truly the peak value is subject to discussion (see 'the edge hypothesis of heterochromatic flicker photometry').

MPOD $=\log \left(R_{\lambda_{s}}^{f} / R_{\lambda_{s}}^{p}\right)-\log \left(R_{\lambda_{\gamma}}^{f} / R_{\lambda_{\gamma}}^{p}\right)$

Formula 1. Example calculation for macular pigment optical density, from Stringham et al. [52]. $R_{\lambda_{s}}^{f}=$ radiance of a peak macular pigment absorption wavelength, e.g. $460 \mathrm{~nm}$, measured at a foveal location. $\mathrm{R}_{\lambda_{\mathrm{S}}}^{\mathrm{p}}=$ radiance of a peak macular pigment absorption wavelength, measured at a parafoveal location, e.g. $7^{\circ} . \mathbf{R}_{\lambda_{\gamma}}^{\dagger}=$ radiance of a negligible macular pigment absorption wavelength, e.g. $570 \mathrm{~nm}$, measured at a foveal location. $\mathbf{R}_{\lambda_{\gamma}}^{\mathrm{p}}=$ radiance of a negligible macular pigment absorption wavelength, measured at a parafoveal location.

\section{$\underline{\text { Instrumentation }}$}

Several variations on MPOD measurement by HFP have been developed since its first use in the 1970s. Traditionally, Maxwellian view devices have been used [e.g. 42, 43, 45]. These are complex optical systems that are not easily portable and which require the use of a dental bite bar. The bite bar keeps the subject's head stable so that their eye is correctly aligned with the incoming light beam $[46,65]$. Given the complexity of these devices, operators need a significant amount of training [46]. As a result, several research groups have simplified the optics and allowed the use of a free view, or Newtonian view, setup [e.g. $46,47,48,62]$. This negates the need for a bite bar, making the procedure more comfortable for the subject. Free view optical systems are also cheaper, easier to operate, and more portable (if not completely portable) than their Maxwellian counterparts [46, 47, 62]. Wooten et al. [46] demonstrated a strong correlation for mean and individual MPOD calculated between their free view system and an established Maxwellian view system $(r=$ 0.95 , no $p$-value provided). This showed that using the free viewing technique does not affect the accuracy of the derived result. Figures 1 and 2 depict a typical Maxwellian view 
optical system and free view optical system, respectively, whilst Table 1 summarizes their differences. It should be noted that slight variations in these differences do occur. For instance, the instrument developed by Beatty and co-workers [47] uses both a quartz halogen and light-emitting diode (LED) light source.

INSERT FIGURES 1 AND 2 ABOUT HERE

INSERT TABLE 1 ABOUT HERE (AFTER FIGURES 1 AND 2)

\section{TEST FIELDS}

The test fields are viewed at a near working distance, e.g. $33 \mathrm{~cm}$ [48]. Most devices use a central stimulus that corresponds to a visual angle of $1^{\circ}$ as standard, although there are exceptions; Landrum's $1.5^{\circ}$ [45] and Werner's $0.70^{\circ}$ [66], for instance. Moreover, many studies have used smaller test stimuli such as $12^{\prime}$ or $30^{\prime}$ when mapping the spatial profile of macular pigment $[42,44,49,50,52,60,64,67-79]$.

The wavelength chosen for the blue light has varied between researchers from $458 \mathrm{~nm}$ [e.g. $11,46,52,75,80,81]$ to $476 \mathrm{~nm}$ [17], and from $530 \mathrm{~nm}$ [e.g. 43, 82-85] to $575 \mathrm{~nm}$ [62] for the green light. Where the blue wavelength does not coincide exactly with the peak of macular pigment absorption, this should be accounted for in the final calculation of MPOD. This is of greater importance in the objective techniques where wavelengths are often further from the peak than those used in HFP. MPOD should also be adjusted according to the bandwidth of the light source; the narrower the bandwidth, the more accurately the measurement reflects MPOD at the particular wavelength [54]. For the HFP device first described by Wooten et al. [46], the LED with peak energy at $458 \mathrm{~nm}$ has a half-bandwidth of $20 \mathrm{~nm}$. As a result, MPOD must be increased by a $15 \%$ constant to correct for this [20, $50,54,77]$.

The peripheral reference measurement is usually made using the same test stimulus as used for the central measure, but the subject's gaze is directed to an eccentric fixation point. An exception to this is the Maculometer (developed by Mellerio et al. [48]) which instead turns the central $1^{\circ}$ field into a fixation point and presents an annular test field at $5.5^{\circ}$ from fixation. The subject therefore fixates centrally throughout the procedure. The authors reported that many subjects found this easier than maintaining an eccentric fixation. The parafoveal location used in different HFP apparatus varies from $4^{\circ}$ from the central fovea [e.g. $11,43,46,62,71,80,81,86]$ to $10^{\circ}[72,76]$ or $12^{\circ}[66]$. Similarly, the location of the peripheral point on the retina varies from temporal, nasal or superior retina, depending on instrument type. 


\section{FLICKER RATES}

The rate at which the blue and green lights are alternated is a difficult decision for researchers to make, since flicker sensitivity can vary between observers [65]. Ideally the flicker rate should allow for a suitable amount of null or minimal flicker to be achieved when adjusting the radiance of the test stimulus. If the flicker frequency is too low for an individual, they will have difficulty obtaining a point of null flicker. Conversely, if the flicker frequency is too high for an individual, they will have a wide range of null flicker, leading to variation in measurements $[50,52,63,75]$. There is also the need for rod and short-wavelength cone suppression to consider (see 'assumptions'). Until recently, most investigators have used set flicker frequencies that have varied from $11 \mathrm{~Hz}$ [e.g. 11, 57, 80, 81, 87, 88] to $30 \mathrm{~Hz}$ [e.g. 49, $68,89,90]$ in the fovea and from $6 \mathrm{~Hz}$ [e.g. $11,46,57,80,81,87,88$ ] to $25 \mathrm{~Hz}$ [e.g. 17, 47, $91]$ in the parafovea.

\section{BACKGROUND FIELDS}

Like test fields, the backgrounds upon which they are presented can also vary in size and wavelength between equipment. Sizes have ranged from $4^{\circ}$ in diameter [e.g. 43,82 ] to $30^{\circ}$ [53]. The colour of the background is invariably a blue wavelength or white. The purpose of these colours is discussed in the 'assumptions' section.

\section{RECENT DEVELOPMENTS}

In 2001, Moreland et al. described a novel method of measuring MPOD by HFP. They used the blue and green phosphor emissions of a colour computer monitor as stimuli for flicker minimization. Although perceptually distinct as blue and green, the broadband emission spectra of the phosphors resulted in a 50\% underestimation of MPOD. This was correctable with a model that incorporated these emission spectra and therefore allowed a way of calibrating the monitor. Bone and Landrum [49] questioned whether the retinal illuminance provided by the instrument was high enough to avoid rod intrusion. Apart from further use by Robson et al. [92] and Robson and Parry [41] this method of MPOD determination does not appear to have been widely used.

Snodderly et al. [50] paved the way for a new customized approach to HFP when they established a standardized protocol for measuring MPOD. The device used was a modified version of the one described by Wooten and colleagues in 1999, and included the addition of optimizing the flicker frequency for each individual. This was achieved by working out each subject's critical flicker frequency at the fovea and parafovea, then using an algorithm to 
determine the appropriate flicker rate to use when measuring MPOD. This procedure has since been adopted in other MPOD research [e.g. 51, 52, 64, 71, 72, 75, 76, 78, 79, 86, 93].

A new HFP technique called the macular assessment profile (MAP) test has been described by Rodriguez-Carmona et al. [94] and Kvansakul et al. [95]. The principle appears similar to the method detailed by Moreland et al. [38] and described above, in so far as the broadband phosphors of a visual display are employed and again require a correction model for this. The authors state that the test is a 'rapid and convenient' way of measuring a subject's macular pigment profile up to $8^{\circ}$ from the fovea, taking advantage of the ability of visual displays to produce stimuli of different sizes at randomized locations [94]. Although the test is said to have been validated, there does not appear to be any formally published data on this.

A further development for customized HFP was briefly described by Engles et al. [93] and elaborated upon by Nolan et al. [75] and Stringham et al. [52]. This involves the inverseyoking of the radiances of the blue and green stimuli so that the overall luminance of the test field remains constant, i.e. when the radiance of the blue stimulus is increased, the radiance of the green stimulus is proportionately decreased. As a result, potential distractions by changes in perceived brightness for the subject are avoided.

An entirely different approach to measurement of MPOD by HFP has been adopted in a new commercially available device which is described in detail by van der Veen et al. [53]. Instead of the subject responding to minimal or no flicker, they respond to the appearance of flicker as the alternation rate is decreased at $6 \mathrm{~Hz}$ per sec from a starting level of $60 \mathrm{~Hz}$. This is above the critical flicker fusion frequency for the test conditions and therefore subjects do not perceive any flicker initially. Rather than the radiance of one wavelength being adjusted by the observer, a sequence of blue-green ratios is used. These are inverse-yoked to ensure that overall luminance stays the same. With similarities to Snodderly et al. [50], the instrument determines each observer's sensitivity to flicker prior to the main part of the test. The technique also offers the possibility of estimating MPOD from a central measure alone, the peripheral measure being estimated from the age of the subject and their expected level of lens yellowing. A comparison between central and peripheral derived MPOD and estimated (central only) MPOD in 5616 eyes revealed a very close correlation $(r=0.92$, no p-value provided) [96]. 
method of HFP MPOD determination, i.e. the radiance of blue light being

increased/decreased until the point of minimal flicker, it takes on a more automated approach, as per the device of van der Veen et al. [53]. The instrument's electronics increase/decrease the amount of blue in the stimulus at a set rate. This removes any interindividual variability in the speed at which the blue-green ratio is adjusted, therefore improving the accuracy of the MPOD value.

\section{Assumptions}

The HFP measurement of MPOD relies on several assumptions. Many of these assumptions are largely accepted because of the close relationship between HFP-derived macular pigment spectral absorption curves and spectral curves derived in vitro. Nevertheless, some of the main assumptions are described:

1. Absorption or scattering properties of the ocular media being accounted for through use of a parafoveal locus $[47,49,56,98]$. Essentially, this means that the amount of yellowing in the media (e.g. the crystalline lens) would influence the measured MPOD value but the reference measure outside of the fovea cancels this effect [96]. This is demonstrated by Formula 2, below. Evidence that this assumption is correct comes from real and simulated data. For instance, Ciulla et al. [81] measured MPOD in 24 patients before and after cataract surgery. No significant difference in MPOD pre or post surgery was found, indicating that varying degrees of crystalline lens absorption does not affect macular pigment measurement when the HFP method is used. Wooten et al. [46] simulated clear and dense lenses by incrementally altering the background field radiance of their free view device. No significant differences in MPOD were found. Most recently, Makridaki et al. [96] demonstrated on a new HFP instrument [53] that lens yellowing, whether simulated or real, had no effect on the measured MPOD.

$B$ fov $x T_{\text {lens }} \times T_{M P}=B$ ref $x T_{\text {lens }}$

$\mathrm{T}_{\mathrm{MP}}=\mathrm{B}$ ref $/ \mathrm{B}$ fov

$M P O D=\log 1 / T_{M P}=\log (B$ fov $/ B$ ref $)$

Formula 2: Macular pigment optical density derivation, from Snodderly and Hammond [65]. In addition to the transmission of blue light through the macular pigment ( $\left.T_{M P}\right)$, the transmission through the lens $\left(T_{\text {lens }}\right)$ is taken into account. $B$ fov and $B$ ref are the radiances 
of blue light needed to minimize flicker at the fovea and reference point, respectively. Since $T_{\text {lens }}$ is assumed to be the same at both the fovea and reference, it is removed from equation (i). The final equation (iii) is a simplified version of Formula 1.

2. Accurate subject fixation and response. This is partly checked through assessment of instrument reliability. Werner et al. [42] also checked fixation accuracy on four of their subjects (age range 15 to 71 ) with an additional test; all subjects were able to accurately fixate to within $\pm 1.00^{\circ}$ of the foveal and parafoveal stimuli, or better, providing further evidence for this particular assumption.

3. Equal spectral sensitivity and distribution of photoreceptors across the retina, such that the difference ratio between the foveal and parafoveal locations is dependent solely on the macular pigment $[12,42,65]$. This assumption is not correct but is accounted for with the design of HFP instrumentation. Rods and short-wavelength sensitive cones (S-cones) are absent at the fovea, whilst being abundant in the peripheral retina and parafovea, respectively [99, 100]. Conversely, medium-wavelength cones (M-cones) and longwavelength cones (L-cones) are present in much higher concentration in the fovea than elsewhere [101]. However, unlike rods and S-cones, the ratio of $M$ to $L$ cones has been shown to remain fairly constant, at least in the central retina [102-104] and, as a result, should not affect the measured MPOD. It is therefore generally accepted that removing the rod and S-cone contributions is of greater importance. To do this, investigators have designed their HFP apparatus accordingly. The background field is often blue to suppress the S-cone population [e.g. 42, 43, 44, 46, 48, 50-52, 62, 105] or bright white to provide photopic conditions and hence suppress the rod population [e.g. 45, 49, 53]. The flicker frequency is chosen so that it is high enough to further exclude rods and S-cones. This is achieved because the flicker rate is above the critical flicker fusion frequency (the alternation rate at which a flickering light is no longer resolvable by the visual system and thus appears steady to an observer) of rods and S-cones, but is still lower than the critical flicker fusion frequency of $\mathrm{M}$ - and L-cones $[65,106,107]$.

4. The peripheral reference locus having a negligible level of macular pigment. Some studies have questioned this assumption [39, 94, 108-112], particularly when eccentricities as little as $4^{\circ}$ from the fovea have been used $[11,20,43,46,62,71,80,81,86-88,113]$. HFP spatial distribution plots of macular pigment (see 'validity and reliability') have gone some way to disproving any concerns, such that for most individuals, the assumption holds.

\section{Validity and reliability}


The validity and reliability of a technique are two important but quite different issues. Reliability, as described by Gallaher et al. [114], refers to the 'ascertainment of the reproducibility of a given measurement on the same subject at two distinct points in time'. Although this is a very important aspect of any instrument, it does not automatically imply that the instrument is valid. This was well illustrated by Snodderly et al. [50], where it was pointed out that one observer had a repeatable negative value of MPOD. The measure was therefore reliable but was nevertheless of questionable validity. For in vivo measurement of MPOD, validity is demonstrated by showing a matching comparison with the known spectral and spatial properties of macular pigment in vitro. Some studies have done this indirectly by comparing the measurements from a new device with those of an older, more established device $[46,56-59]$.

\section{VALIDITY}

As mentioned above, the validity of MPOD measurement by HFP can be assessed in two main ways $[52,71]$. Either by deriving the spatial profile of macular pigment across the fovea [e.g. $42,44,50,52,53,67,70,71,75]$ and comparing that to in vitro knowledge of macular pigment distribution [e.g. 5, 7, 24, 115], or by deriving the spectral absorption profile of macular pigment $[17,42,43,47,49,52,71]$ and comparing that to the shape of a known in vitro absorption curve of macular pigment. The latter method is considered to be more robust than the former, since spatial profile can vary between individuals $[44,50,59,64,71$, $75,78,116,117]$.

Spatial profiles of MPOD are achieved by altering the size and/or the eccentric position of the test stimulus, thus producing a curve that can be used to describe the change in MPOD with increasing eccentricity from the central fovea. An example is shown in Figure 3.

\section{INSERT FIGURE 3 ABOUT HERE}

Spectral absorption profiles of MPOD are obtained by systematically altering the wavelength of the test stimulus. The procedure is the same as that used to determine peak MPOD, except that a number of light wavelengths must be alternated with the reference stimulus until minimum flicker is accomplished, instead of the blue light alone. The extra measurements increase the duration of the test but permit a curve of spectral absorption to be plotted, see Figure 4. The in vivo HFP-generated curve is simultaneously compared with the shape of an in vitro spectral curve of lutein and zeaxanthin. The choice of which in vitro curve to use is not an easy one, since the ideal comparison of in situ spectral data from 
human retinas does not currently exist [54]. Consequently, different researchers have used different data. These include: Wyszecki and Stiles' composite data curve ${ }^{1}$ [118], used by Werner et al. [42], Hammond and Fuld [43], Beatty et al. [47], and Beatty et al. [17]; Snodderly and colleagues' microspectrophotometry-derived data from primate monkeys [6], used by Hammond and Fuld [43]; and spectral measures of lutein and zeaxanthin dissolved in olive oil [24] or incorporated in liposomes [9]. These last two in vitro spectrums have more recently been used in combination when assessing validity $[52,54,71]$.

The outcomes of these validity tests have shown a good correlation with in vitro MPOD distribution and absorption [e.g. 71] and therefore attest to the validity of HFP as a measurement method for MPOD.

INSERT FIGURE 4 ABOUT HERE

${ }^{1}$ The data from Wyszecki and Stiles [118] is actually a weighted composite curve of six psychophysical methods that had been used up to that time, rather than in vitro data. 
RELIABILITY

Numerous researchers, through test-retest checks, have assessed the reliability of HFP measurement of macular pigment. The statistical descriptors used to evaluate reliability vary between studies. Table 2 is a summary of all available information on HFP test-retest reliability indicators. It shows that for most subjects, HFP provides repeatable measures of MPOD and is therefore a reliable technique. Interestingly, the two most recent evaluations of HFP appear to give the weakest indication of its reliability $[119,120]$. These studies were independent of each other but used the same HFP device, one of the first that has been designed for use in a clinical setting rather than a research setting. The results suggest that further developments may be required for this particular HFP instrument in order to verify its suitability to accurately assess MPOD.

\section{INSERT TABLE 2 ABOUT HERE}

\section{The edge hypothesis of heterochromatic flicker photometry}

As mentioned previously, most instruments use a stimulus size of $1^{\circ}$ to measure MPOD. One might reasonably assume that this measures the total amount of macular pigment across the whole $1^{\circ}$ area or the peak level of macular pigment. However, many investigators disagree with both these assumptions and instead believe that the level of calculated MPOD is mediated by the edge of the stimulus [e.g. $11,42,44,48,50,52,121$ ], so that for a $1^{\circ}$ stimulus, the recorded MPOD corresponds to the macular pigment level at $0.5^{\circ}$ from the centre of the fovea. Werner et al. [42] were the first to suggest this theory and it gained momentum when Hammond et al. [44] found a very high correlation $(r=0.91, p<0.00001)$ between MPOD measured with a $1^{\circ}$ test stimulus and a point test stimulus of $12^{\prime}$ placed at $0.5^{\circ}$ (see Figure 5). Their data also indicated that MPOD at $0.5^{\circ}$ (as measured with a $1^{\circ}$ stimulus) is an estimated $69 \%$ of the true peak MPOD. For example, an MPOD value of 0.4 measured with a $1^{\circ}$ test would indicate a peak macular pigment density of 0.58 . The 'edge hypothesis' has been questioned, however, most notably by Bone et al. [68]. In contrast to Hammond et al. [44], they found that the measured MPOD corresponds to the level of macular pigment at approximately $50 \%$ of the stimulus radius (Figure 6). Further evaluation indicated that this equated closely to the average amount of macular pigment over the whole stimulus area [68]. Nevertheless, in spite of this conflicting evidence, most researchers have continued to assume the edge hypothesis in their HFP work [e.g. 60, 63, 64, 73-75, 78, 122] and this would appear to be a reasonable decision, with further evidence for it coming most recently from van der Veen et al.[121]. 
INSERT FIGURES 5 AND 6 ABOUT HERE

\section{Advantages and disadvantages of HFP}

Advantages include: 1) no pupil dilation required 2) inexpensive equipment relative to objective techniques 3 ) independence from absorption and scattering properties of the ocular media 4) good test-retest reliability on many subject populations 5) proven validity. Disadvantages include: 1) some subjects finding HFP difficult to carry out, especially the peripheral task, which is subject to Troxler's effect - a perceptual fading of peripheral stimuli [123]; Troxler's effect also becomes more distracting the more eccentric the peripheral target is, making the use of eccentricities certain to have no macular pigment difficult to record accurately 2) a long testing time if complete spectral and/or spatial distribution is required 3) unsuitability for some individuals, such as young children, people with learning difficulties or people with insufficient visual acuity or visual fields.

\section{Motion photometry}

The minimum motion paradigm was initially described by Stumpf [124], although this went largely unnoticed until its translation into English by Todorović in 1996 [40, 125]. With parallels to HFP, it refers to the perceived reduction in motion of a moving square or sine wave grating as equiluminance of the colours involved is reached. The concept was taken up for use in photometry by both Moreland [126, 127] and Anstis and Cavanagh [128], but in subtly different ways. This then led to the use of minimum motion photometry for in vivo measurement of macular pigment [e.g. 33, 37].

\section{Procedure}

Many of the principles described for HFP also apply for motion photometry measurement of MPOD, i.e. a wavelength of light at the peak of macular pigment absorption is compared with a wavelength of light not absorbed by macular pigment, at central and parafoveal locations. Moving square wave gratings are used, with the bars being alternately illuminated by the two light wavelengths. The radiance of the longer wavelength stimulus is adjusted until the motion appears to slow down or change direction, depending on the method being employed [126-128]. The slowing down of the grating is minimum motion photometry, whereas the reversal of grating movement is known as apparent motion photometry [40]. As with HFP, different radiances of the test wavelength will be required for equiluminance at the 
foveal and parafoveal positions, on account of the higher levels of macular pigment at the fovea. A log ratio of these radiances provides a measure of MPOD [37-41, 92, 129].

\section{$\underline{\text { Instrumentation }}$}

The motion photometry technique for measurement of MPOD has not been as widely used as HFP. Apart from the fundamental differences between classic minimum motion photometry and apparent motion photometry (explained below), there is little variation in the instrumentation that has been adopted.

\section{MINIMUM MOTION PHOTOMETRY}

Minimum motion photometry for MPOD, as described and used by Moreland, Robson and colleagues [e.g. 37, 38-41, 92, 129], employs a Moreland anomaloscope (traditionally used for colour vision assessment) that is adapted to produce a moving square wave grating with a spatial frequency of 0.38 cycles per degree. A rotating spiral mirror generates the grating which, when viewed through a circular or annular stop, appears to move horizontally across the visual field. The bars of the grating are alternately illuminated with two narrow-band interference filters from a single tungsten-halogen lamp. The interference filters typically provide wavelengths of $460 \mathrm{~nm}$ (blue - maximal absorption by macular pigment) and 580 $\mathrm{nm}$ (yellow - negligible absorption by macular pigment). Luminance matched filters of 450 $\mathrm{nm}$ are added to the grating bars to create a background pedestal that saturates S-cones (see 'assumptions'). The grating moves at a constant velocity of $14 \mathrm{~Hz}$; this also rules out any rod or S-cone contribution.

Unlike HFP, it is the norm with motion photometry for the spatial profile of macular pigment to be plotted, rather than peak MPOD alone. Consequently, the test fields comprise up to two central, circular stops of $0.8-0.9^{\circ}$ (visual angle) and $2.2^{\circ}$, and 11 annular stops placed eccentrically from $0.8^{\circ}-7.5^{\circ}$ in the superior visual field. The setup is illustrated in Figure 7. The minimum motion technique described here uses a Maxwellian view system but does not require a bite bar; rather, an adjustable chin rest (vertically and horizontally) is used for pupil centration [40].

\section{INSERT FIGURE 7 ABOUT HERE}

\section{APPARENT MOTION PHOTOMETRY}


Apparent motion photometry, based on the phenomenon detailed by Anstis and Cavanagh [128], has been developed into a commercially available device for MPOD assessment by West and Mellerio [130]. However, there do not appear to be any peer-reviewed studies on the instrument. The instrumentation details given below are therefore based on information from the Cambridge Research Systems' (CRS) website (http://www.crsltd.com/catalog/metropsis/MP.html).

Instead of a Moreland anomaloscope, a cathode ray tube (CRT) monitor is used. Four square wave gratings on a blue background are presented sequentially, 90 degrees out of phase with each other. The first and third gratings are made up of blue and red bars, produced by the blue and red phosphors of the CRT; blue for maximal macular pigment and red for negligible macular pigment absorption. The second and fourth gratings are achromatic, being composed of light and dark grey bars, i.e. luminance gratings. The apparent motion paradigm dictates that when the luminance of the red bars is greater than the blue, the red bars appear to jump rightwards to the light grey bars in the luminance grating, and when the luminance of the blue bars is greater than the red, the blue bars appear to jump leftwards to the light grey bars in the luminance grating (Figure 8, left). The subject therefore sees movement in one of two directions. The setup of West and Mellerio's device results in these directions being up or down (Figure 8, right). The red luminance is adjustable, and at the point of red-blue equiluminance, the direction of motion becomes 'ambiguous'. A two-alternative forced choice procedure is used to determine equiluminance (and subsequent MPOD) at central and eccentric locations. The system crosses over the threshold several times and can provide the standard deviation of each MPOD measurement. The grating alternation frequency must be optimized, usually between 8 and $20 \mathrm{~Hz}$, for each subject for reliable results.

\section{INSERT FIGURE 8 ABOUT HERE}

Like minimum motion, the apparent motion photometer for MPOD routinely plots the spatial distribution of macular pigment. Two central vertical strips $\left(0.3^{\circ} \times 1.25^{\circ}\right)$ are located at $0^{\circ}$ and $1^{\circ}$, whilst six $45^{\circ}$ annular test fields are located from $2^{\circ}-7^{\circ}$ from fixation. A shorter threelocation test is also available.

With a CRT monitor comes the problem of spectral overlap of the red, green and blue phosphors, similar to the problems of the colour monitor for HFP discussed in HFP 'recent developments' $[38,41,92]$. The apparent motion photometer overcomes this with the use of 
an optical filter that blocks light between 460 and $640 \mathrm{~nm}$, thus avoiding a significant underestimate of MPOD.

\section{Assumptions}

The same assumptions apply to minimum motion photometry as for HFP, namely absorption or scattering properties of the ocular media being accounted for through use of a parafoveal locus; accurate subject fixation and response; equal spectral sensitivity and distribution of Land $\mathrm{M}$-cones across the retina; and the peripheral reference locus having a negligible level of macular pigment.

The apparent motion device offers an interesting solution to accurate fixation by using a video gaze tracking system that inhibits stimulus presentation whenever fixation is not maintained to a sufficient level (within $\pm 0.5^{\circ}$ of target). This offers an improvement over unmonitored free view techniques whilst avoiding the need for a Maxwellian view system. With respect to L- and M-cone distribution, Robson et al. [129] used a 460/550 nm combination as well as the customary $460 / 580 \mathrm{~nm}$ to see if there was any variation in MPOD measurements. The correlation was extremely strong (slope $=1.00, r=0.99$ ), indicating that even if the distribution does vary with eccentricity, the effect is likely to be very small [129]. Like HFP, motion photometry also uses a blue background and a suitable temporal frequency to ensure rod and S-cone suppression. The assumption of the peripheral reference eccentricity having negligible macular pigment should hold for most individuals because spatial distribution is always plotted out as far as 7 or 8 degrees with motion photometry.

\section{Validity}

We could find no evidence of motion photometry being used to derive spectral profiles of macular pigment. As a result, it is difficult to know the true validity of the technique, although average MPOD values and spatial profiles are in line with HFP, and consistent correlations with the autofluorescence method of macular pigment measurement [39, 129] and two HFP devices $[38,48]$ have been found. Nevertheless, questions regarding the validity of motion photometry for measurement of macular pigment have been raised [54].

$\underline{\text { Reliability }}$ 
The reliability of minimum and apparent motion photometry has not been as vigorously assessed as it has been with HFP. The average of five readings is taken at each location $[37,39,41,92,129]$ and repeated measurements have been incorporated in averaged results [39]. However, there does not appear to be any published statistical data on testretest reliability.

\section{The non-edge hypothesis of motion photometry}

It was explained earlier that most researchers using HFP assume an edge hypothesis with MPOD. In minimum motion photometry, data analysis has led to the support of a non-edge hypothesis $[34,39,40]$. To reiterate, this would mean that when a circular, foveal stimulus is used, the measured MPOD would not represent the amount of macular pigment at the stimulus radius. In the case of motion photometry, researchers believe that the measured MPOD actually represents the amount of macular pigment at approximately $70 \%$ of the stimulus radius $[34,40]$. Subsequently, the 'peak' MPOD value using the smaller 0.8-0.9 central stimulus has been plotted at $0.3^{\circ}$ eccentricity from the fovea [e.g. 39, 41, 129].

\section{Advantages and disadvantages of motion photometry}

As a psychophysical technique, motion photometry has much in common with HFP in terms of pros and cons. There is no need for pupil dilation, any interference from the ocular media is accounted for and it is a relatively straightforward test for subjects to partake in. On the down side, Troxler's effect remains a problem for some individuals, and good comprehension of the task is required, so it is not suitable for everyone. No information is provided in any motion photometry studies regarding the level of visual acuity of subjects; therefore it is unknown whether reliable MPOD measurements are achievable on subjects with lower than normal acuity.

\section{Objective techniques}

The objective techniques for measuring MPOD are:

Fundus reflectometry

Fundus autofluorescence

Resonance Raman spectroscopy

Electrophysiology using visual evoked potentials 


\section{Fundus reflectometry}

Quantitative measurement of light reflected from the fundus is known as fundus reflectometry (FR), and the researchers Brindley and Willmer [131] were the first to adopt this technique. Their aim was to estimate MPOD in vivo by comparing light reflected at the macula with light reflected from a peripheral area of retina. Since then, FR has gone on to become the most widely used of the objective methods for MPOD measurement, although many improvements and variations have been developed along the way.

\section{Procedure}

When light enters the eye it has many structures to pass through, including the cornea, the crystalline lens, the retina and the choroid. Some of these structures (and their components) will reflect a small part of the light, whilst others will absorb part of it. Through measurement of reflected light from the retina and choroid, FR is able to assess several ocular features, including macular pigment [109]. A thorough history of FR is provided by Berendschot, DeLint and van Norren [132]; this review will be limited to the use of FR in measuring MPOD.

Although there are several variations on the reflectometry procedure, there are two methods that predominate. The first is a comparison technique, similar to that used in HFP. Light reflected from the fovea is compared with light reflected from an eccentric retinal area, using two wavelengths (one absorbed by macular pigment and one not) or using a spectrum of wavelengths. Since macular pigment absorbs rather than reflects certain wavelengths there will be a difference in the observed reflectances at the fovea and periphery, owing to the assumed lack of macular pigment at the eccentric site. Researchers who have used this method include Brindley and Willmer [131], van Norren and Tiemeijer [133], Delori and Pflibsen [134], Elsner et al. [135], Berendschot et al. [136], Delori et al. [56], Bour et al. [137], Wüstemeyer et al. [98], and Cardinault et al. [138].

The second core technique is known as a spectral analysis [109]. As the name suggests, this involves the analysis of a spectrum of reflected light from a spot of light on the retina. To achieve this, a detailed optical model of the pathways of light in the eye is required. $A$ number of optical models of increasing complexity have been proposed over the years, from van Norren and Tiemeijer [133] through to van de Kraats and van Norren [139]. Probably the most familiar optical model is that derived by van de Kraats, Berendschot and van Norren [140], which has been used to work out MPOD in several studies [e.g. 58, 136, 141-144]. In 
essence, the density of macular pigment is determined using its known spectral characteristics and by taking into account the amount of light reflected at the internal limiting membrane, the photoreceptor discs and the sclera [58, 136, 140, 141, 143]. The densities of the lens, melanin and blood are likewise calculated.

Even with these two quite separate forms of FR, there is often an overlap between the two, as demonstrated in the following section.

\section{$\underline{\text { Instrumentation }}$}

Many instruments have been used for FR and it is beyond the scope of this review to explain them all. Consequently, only the more recent fundus reflectometers will be described in any detail. However, common to most methods of FR is the need for pupil dilation, a bleaching of the visual pigments prior to measurement, and some form of head stabilization, either with a bite bar or with a chin rest and temple pads.

Equipment for reflectometry can be broadly categorised into modified fundus cameras, purpose-built reflectometers and modified scanning laser ophthalmoscopes.

\section{FUNDUS CAMERAS}

Many investigators have used modified fundus cameras to measure MPOD, including van Norren and Tiemeijer [133], Delori and Pflibsen [134], Chen, Chang and Wu [108], Bour et al. [137], Chang et al. [145], Neuringer et al. [3], and Bone, Brener and Gibert [59]. Of these, some have used the comparison technique, some spectral analysis and others a mixture of the two. Chen et al. [108], for example, used an optical model as per the spectral analysis technique but only two wavelengths $(460 \mathrm{~nm}$ and $560 \mathrm{~nm}$ ) rather than a full spectrum of wavelengths. In brief, their setup, like several others, consisted of a fundus camera connected to a cooled CCD (charge-coupled device). The filter normally used to take redfree photographs was replaced with narrow band interference filters of 460 and $560 \mathrm{~nm}$, i.e. maximal and minimal macular pigment absorption. Following pupil dilation, the subjects were instructed to fixate a dim red dot with the eye not being tested whilst the measured eye was slowly light adapted in order to bleach nearly all the photoreceptor pigments [56, 108, 146]. Two fundus pictures, one taken at each of the two wavelengths, were manually aligned using retinal landmarks. Using a chosen optical model, MPOD at each pixel point in the retina was calculated. In this way, Chen et al. were also able to plot the spatial distribution of macular pigment across the central retina. 
Recently, Bone et al. [59] described a modified fundus camera that does not require pupil dilation or bleaching of the photoreceptor pigments.

\section{PURPOSE-BUILT REFLECTOMETERS}

The most recent 'purpose-built reflectometers' are the Foveal Reflection Analyzer (FRA), originally developed by Zagers et al. [146] and the Macular Pigment Reflectometer (MPR), originally developed by van de Kraats et al. [117].

The diagrammatic setup of the FRA is shown in Figure 9. After dilation, subjects fixate a central cross hair (Figure 9b). In the original instrument (FRA 1), light from a halogen lamp is directed into the eye as a Maxwellian view system with an entrance pupil of $0.8 \times 1.2 \mathrm{~mm}$. This illuminates a $2.8^{\circ}$ spot on the central fovea, of which the middle $1.9^{\circ}$ is used for analysis. A video observation channel of the pupil and retina helps alignment as well as allowing monitoring of subject fixation. An imaging spectrograph collects the reflected light from the $1.9^{\circ}$ area and focuses it onto a cooled CCD camera. The spectrograph has a slit that creates a $0.8 \times 12 \mathrm{~mm}$ exit pupil above the smaller entrance pupil, and its spectral range is 420 to $790 \mathrm{~nm}$, thus allowing a spectral analysis of the reflected light using one of the optical models referred to earlier. The FRA 1 has been used to investigate MPOD in studies by Zagers and van Norren [147] and Berendschot and van Norren [141]. Berendschot and van Norren [141] also used a newer version of the device, the FRA 2, which has a number of differences from the first version, including being smaller, which makes it desktop mountable. Kanis et al. [143, 148] and van de Kraats and van Norren [139] have also used the FRA 2 in their studies.

\section{INSERT FIGURE 9 ABOUT HERE}

The diagrammatic setup of the MPR is shown in Figure 10. Like the FRA, the MPR, as described by van de Kraats et al. [58], involves a 30W halogen lamp directed into the pupil, a separated exit pupil and a spectrometer, i.e. spectrograph. The spectrometer has a spectral range of 400 to $800 \mathrm{~nm}$. The subject is asked to fixate the centre of a $1^{\circ}$ incoming light beam. This incoming illumination forms a $1^{\circ}$ spot on the central fovea; the reflected light is also collected over the same $1^{\circ}$ area and analyzed using an optical model. The MPR is the first reflectometry device that has a proven ability to measure MPOD through an undilated pupil, provided the pupil is $3 \mathrm{~mm}$ or larger; van de Kraats et al. [58] found no significant difference between their MPOD measurements for dilated and undilated pupils in 20 subjects. A further development of the MPR, recently reported by van de Kraats et al. 
[139], is the means to measure the individual optical densities of lutein and zeaxanthin, the components of macular pigment.

\section{INSERT FIGURE 10 ABOUT HERE}

\section{SCANNING LASER OPHTHALMOSCOPES}

Elsner et al. [149] were the first to use a scanning laser ophthalmoscope (SLO) for the purpose of measuring MPOD. Since then it has become a popular FR method for measuring macular pigment [e.g. 25, 98, 116, 136, 141, 150]. Some SLOs have been custom-built for MPOD measurement and as such are not accessible to most clinicians [135, 136]. However, Wüstemeyer et al. [98] modified a commercially available SLO, allowing reflectance images to be recorded with an argon laser at wavelengths $488 \mathrm{~nm}$ and $514 \mathrm{~nm}$, with a fast switch between the two. They used the comparison technique, with an eccentric reference point of $14^{\circ}$ from the fovea. MPOD in a $2^{\circ}$ central fovea test field was calculated as follows (Formula 3):

MPOD $=\mathrm{C}_{\lambda}{ }^{*}\left[\log \left(\operatorname{Ref}_{514, \text { foveal }} / \operatorname{Ref}_{488, \text { foveal }}\right)-\log \left(\operatorname{Ref}_{514, \text { parafoveal }} / \operatorname{Ref}_{488, \text { parafoveal }}\right)\right]$

Formula 3. Calculation of macular pigment optical density, from Wüstemeyer et al. [98]. $C_{\lambda}=$ constant, dependent on the absorption coefficients of macular pigment. $\operatorname{Ref}_{514}$ and $\operatorname{Ref}_{488}=$ reflectances measured at 514 and $488 \mathrm{~nm}$.

Using a foveal and parafoveal comparison is not common to all SLOs when measuring MPOD. Berendschot and van Norren [116, 141], for instance, used the same two wavelengths as Wüstemeyer et al. [98] but did not use any specific eccentric reference point and therefore produced density maps of the sum of both the lens and macular pigment.

One of the main advantages of using a SLO over other FR techniques is its confocal optics, which help minimize stray light scatter, the biggest hindrance in FR. This will be elaborated on in the 'assumptions' section.

\section{TEST FIELD VARIATIONS}

The size of the detection field chosen to measure peak MPOD varies not only between the three categories of reflectometer but also within the categories, from $0.5^{\circ}[116,141]$ to $2^{\circ}$ $[25,56,98,138]$ and $2.5^{\circ}$ [133]. Likewise, when two wavelengths corresponding to high and low macular pigment absorption are used, as is often the case for modified fundus cameras, the chosen wavelengths differ slightly between equipment, as they do in HFP devices. In 
SLOs, the two wavelengths are always 488 and $514 \mathrm{~nm}$ because these are the two most appropriate argon laser lines. The deviation of these laser lines from the true maximum and minimum of macular pigment absorption ( 460 and $>530 \mathrm{~nm}$, respectively [54]) requires that a correction is made to account for this in the final MPOD estimation [116, 136, 141, 151], although it is not clear whether all research groups actually do this. For the comparison technique when a peripheral reference point is used, the chosen eccentricity has ranged from as little as $4^{\circ}$ from the central fovea [137] up to $14^{\circ}[98,136]$.

\section{Assumptions}

The assumptions for FR are not as openly explained in the literature as for HFP. Nonetheless, several of the more commonly noted assumptions are highlighted below:

1. Homogeneity of fundus tissues. The spectral characteristics, absorption, reflection and scattering properties of the various retinal tissues (e.g. melanin) are assumed to be homogenous across the areas being assessed. Gellermann and Bernstein [111], among others, point out that this is a simplification. However, most researchers [e.g. 58] do not consider this to be a problem, and with good reason it would seem. For instance, the effect of irregular RPE melanin distribution on measured MPOD was investigated by Delori et al. [56]. They concluded that it had no strong effect on MPOD as measured with their reflectometry technique.

2. Bleaching of photoreceptor pigments. It has been established that $93-99 \%$ of cone photopigment and $59-85 \%$ of rod photopigment is bleached as a result of the level of illumination used prior to measurement, depending on the particular reflectometry method used $[56,58,108,137]$. Bleaching is important to avoid light absorption by the pigments and their subsequent interference with MPOD. It is assumed that any remaining unbleached photopigment, particularly rhodopsin (the pigment in rods), has a minimal effect. This has been investigated by Chen et al. [108], Delori et al. [56] and Bour et al. [137] and proven to be the case.

3. Light scatter accounted for. If reflectance from pre-retinal and intra-retinal structures are not controlled for, the measured MPOD can be artificially low [56, 109, 141]. This is because the reflectance method works on the principle that all the incident light is reflected after passing through the macular pigment. If some light is reflected before it reaches the macular pigment, e.g. by the crystalline lens, then this will be collected as reflected light but it won't actually have been affected by macular pigment absorption, thus leading to an erroneously 
low MPOD [25]. Most reflectometry devices do aim to eliminate this problem, although it would appear that some are more successful than others, judging by the lower than 'average' MPOD estimates found in some studies [e.g. 25, 56, 98, 137]. Methods used to allow this assumption to hold as far as possible include separating the entrance and exit pupils, using confocal optics as found in SLOs, and the incorporation of stray light into optical models. In addition, for the comparison technique of $\mathrm{FR}$, the use of a peripheral reference should account for crystalline lens scatter [56].

4. Negligible macular pigment at a peripheral reference site. If the comparison method is used, the same rules apply as per HFP; provided the peripheral locus is eccentric enough to exclude any macular pigment contribution, the assumption will hold. Choosing a point far enough away from the fovea is easier with FR, since there is little participation required by the subject. Delori et al. [56] commented that the use of a peripheral reference in FR is enough to reduce the influence of the ocular media on the MPOD measurement. Hammond et al. [54], however, argue that regardless of this, as an objective technique FR will suffer from a loss of signal as a result of increased lens scatter and density in some subjects.

5. MPOD measured over the entire stimulus area. Whereas with the psychophysical methods there is some disagreement regarding which part of the macular pigment distribution is actually being measured with the test stimulus, with FR there is a general consensus that the MPOD is the mean amount over the chosen detection field [56, 98, 136, 141]. However, this assumption has not been verified [54].

\section{Validity}

Like HFP, the validity of FR for measurement of MPOD can be assessed by plotting spectral and spatial profiles of macular pigment and comparing these with in vitro data. Van de Kraats et al. [58] argue that any technique demonstrating an increase in MPOD following increased lutein intake is also an indication of its validity. Several reflectometry studies have shown such an increase $[3,136,152]$.

Spectral profiles of macular pigment have not been generated to the extent that they have in HFP studies. In fact, there appears to be only one study that has comprehensively investigated this aspect of validity. Delori et al. [56], used the comparison technique and measured reflectance at wavelengths of $430,450,470,490,520$ and $550 \mathrm{~nm}$. Their results from 147 subjects accurately matched the spectral curve of macular pigment in vitro, albeit with some small systematic deviations such as lower values at $430 \mathrm{~nm}$ (see Figure 12). The 
investigators point out, however, that the deviations altered depending on which in vitro curve they chose for comparison, highlighting the point that the true macular pigment absorption spectrum is not known with enough certainty to assume that the reflectometry deviations are an inaccuracy. It is also interesting to note that lower values at the short wavelength end of the spectrum are also a common finding in HFP spectral profiles (see Figure 4), perhaps lending further support to there being a genuine difference between the in vivo and in vitro macular pigment spectral profile.

The plotting of 'macular pigment maps' to assess macular pigment spatial profiles has become reasonably commonplace in FR studies [e.g. 59, 108, 116, 135, 136, 137]. For SLOs, this generally involves a digital subtraction of the images obtained at two wavelengths (maximal and minimal macular pigment absorption). Chen et al. [108], used a modified fundus camera (the method is described above) and obtained spatial distributions for 54 subjects of various ages. These distributions, divided into three age groups, are shown in Figure 11. The decline in macular pigment from the fovea is rapid and symmetrical, very similar to the decline in macular pigment expected from in vitro knowledge and also from HFP-derived plots of MPOD (see Figure 3).

\section{INSERT FIGURE 11 ABOUT HERE}

Chen et al. [108] looked at the half width of macular pigment distribution (HWMPD) for each of the age groups, and found a significant increase (i.e. widening) with age. With further analyses they also noted that 'shoulders' of varying type were present in the MPOD profile of all subjects. Small irregularities in the otherwise undisturbed decline of macular pigment with eccentricity have been reported in other studies [5, 44, 59, 64, 71, 75, 115-117, 153, 154], both in vivo and in vitro, and are the subject of much ongoing discussion [e.g. 155].

Recently, the MPR [58] has been used to investigate MPOD distribution, or more specifically, to plot the individual distributions of lutein and zeaxanthin [152]. Rather than macular pigment maps, reflection spectra were taken at a variety of eccentricities up to $8^{\circ}$ from the fovea, in a similar manner to HFP.

An indirect way of demonstrating validity is to compare results with those of a technique with established validity, i.e. HFP. This has been done in several FR studies with fairly good results $[56,58,59,141]$.

\section{$\underline{\text { Reliability }}$}


With so many different instruments being used to measure MPOD by $\mathrm{FR}$, there ought to be an abundance of reliability data available. However, few studies have assessed the intersession reliability of their devices, although more have assessed within-session reliability. This is perhaps because, unlike HFP, the actual measurement time in FR is short and does not demand too much effort from the subject; hence it's more convenient to take repeat measurements within the same session. That said, Zagers et al. [146] believed the variability in their intra-session MPOD results was the result of fixation errors, with the less experienced subjects showing greater variability. Nonetheless, as Snodderly and colleagues point out, inter-session reliability is really more valuable than intra-session reliability [50]. Since results generally show higher variability between sessions, this is a more robust test for an instrument. Table 3 outlines the reliability indicators provided in FR studies regarding instrument reliability. It shows that for the studies with published data on reliability, the results are good and comparable with HFP.

\section{INSERT TABLE 3 ABOUT HERE}

\section{Advantages and disadvantages of FR}

Advantages include: 1) as an objective method it requires minimal effort from the subject 2) quick measurement time 3 ) density maps of macular pigment distribution can be plotted quickly 4) reliability appears to be good in several instruments 5) suitability for many subject populations including children. Disadvantages include: 1) pupil dilation normally required 2) the need for precise alignment before measurements 3 ) unpleasant light levels because of the requirement for photopigment bleaching 4 ) the need to control for light scatter, which can include considerable modeling 5 ) costly and complicated instruments, although attempts are being made to produce less expensive reflectometers using commonly available equipment $[59,98,137]$.

\section{Fundus autofluorescence}

One of the newer ways for measuring MPOD in vivo relies on the intrinsic fluorescence, or autofluorescence (AF), of lipofuscin in the retinal pigment epithelium (RPE). Lipofuscin in the RPE is a waste product of photoreceptor outer segment phagocytosis and it accumulates with age [156-158]. When excited with light wavelengths of 400 to $590 \mathrm{~nm}$, lipofuscin fluoresces, emitting light in the wavelength range $520-800 \mathrm{~nm}$ [159]. 
Delori [156] was the first to develop a technique for fundus AF with the primary aim of measuring lipofuscin. Further studies by Delori et al. [157] and von Rückmann et al. [158] provided evidence for lipofuscin being the main fluorophore in AF. It was their observations of a decrease in AF at the macula that lead to the use of $A F$ as a means for measuring MPOD. For an in-depth look at fundus AF and its application, see Schmitz-Valckenberg et al. [160]. The current review will concentrate on the use of AF in macular pigment measurement.

\section{Procedure}

To recall, the absorption spectrum of macular pigment is in the range $400-540 \mathrm{~nm}$ [6] and the absorption spectrum of lipofuscin is in the range $400-590 \mathrm{~nm}$ [159]. Since macular pigment is located anterior to lipofuscin, incoming light directed the fovea will be absorbed by the macular pigment before it reaches the lipofuscin, provided the wavelength of the light is within the absorption range of macular pigment. As a result, there will be an attenuation of lipofuscin fluorescence at the macula; the more macular pigment present, the higher the level of attenuation. By comparing the emitted AF at the fovea and parafovea of two excitation wavelengths, one that is well absorbed by macular pigment and one that is not, MPOD can be calculated [56].

Two AF procedures exist for measurement of macular pigment. The first is a comparison method as used in HFP and some forms of FR. The emitted fluorescence is collected from a foveal and parafoveal sampling area, and then compared to give a measure of MPOD [e.g. 56]. The second and more common procedure is an imaging method whereby up to 32 images $[39,129]$ are taken in succession with one or two wavelengths. The images are aligned (manually or using dedicated software) and averaged, then a greyscale index of intensity is used to generate density maps of macular pigment, which includes a measure of peak MPOD. Key studies using the AF imaging technique include those of Wüstemeyer et al. [25], Berendschot and van Norren [141], Delori et al. [117], Liew et al. [161], Trieschmann et al. [162] and Wolf-Schnurrbusch et al. [154].

\section{$\underline{\text { Instrumentation }}$}

By far the most commonly used instrument for AF acquisition of MPOD is the confocal SLO, purpose-built [e.g. 116, 141] or a modified version of a commercially available SLO [e.g. 25, 161, 163]. All SLOs use the imaging method of fundus AF. The subject fixes a target whilst multiple AF fundus images, usually taken over a $20^{\circ}$ field, are obtained at wavelengths of 
$488 \mathrm{~nm}$ and $514 \mathrm{~nm}$. A barrier filter above or close to the threshold of MP absorption (e.g. $530 \mathrm{~nm}$ ) is used to ensure that the emitted AF is only collected outside the absorption range of macular pigment, thereby avoiding any further absorption and allowing a single-pass measurement rather than a double-pass as used in reflectometry. All the AF images are aligned and averaged for each wavelength. A computer program (see Trieschmann et al. [162] for details) digitally subtracts the averaged images at the two wavelengths and uses a greyscale index of intensity to create a map of MPOD. A foveal MPOD value is calculated at a point eccentricity $[117,161,164]$ or within a certain area centred on the fovea $[25,154$, 161, 163]. As in FR, a correction should be made when using SLOs, to account for the argon laser lines not coinciding exactly with the maximum and minimum wavelengths of macular pigment absorption [26, 39, 141, 151, 153, 164].

Other equipment that has been used to assess MPOD using AF includes the fundus fluorometer/spectrophotometer (first described by Delori [156] and used specifically for macular pigment measurement by Delori et al. [56]) and a modified fundus camera [117, 159]. The fundus fluorometer employs the comparison method. A number of different wavelengths are directed at a $3^{\circ}$ retinal area and the fluorescence is collected from a $2^{\circ}$ sampling field concentric within the $3^{\circ}$ area [56]. The subject is asked to fixate centrally or at $7^{\circ}$ in order to obtain emission data from the fovea and parafovea. MPOD is then calculated using the foveal and parafoveal AF information at excitation wavelengths of $470 \mathrm{~nm}$ and 550 $\mathrm{nm}$. In contrast, the modified fundus camera employs the imaging method. The camera is coupled to a cooled CCD camera and takes pictures of a $15^{\circ}$ retinal field using wavelengths of 470 and $545 \mathrm{~nm}$. At this point the technique becomes very similar to that of SLO AF imaging, i.e. image alignment and analysis by a computer program, thus providing macular pigment density maps, including a measure of peak MPOD [117, 159].

Common to all forms of AF instrumentation is the need for bleaching of the visual pigments prior to measurement (see 'assumptions') and pupil dilation, although a non-mydriatic version has been described [153].

\section{One-wavelength versus two-wavelength AF}

The vast majority of AF-based macular pigment studies have used two wavelengths (corresponding to high and low macular pigment absorption) to derive MPOD. There have been a handful of studies, however, that have used only the high absorption wavelength [19, $39,129]$. It is then presumed that any reduction in AF across the imaged area is due entirely to the presence of macular pigment [19]. Whilst some good correlations between MPOD 
measured with one-wavelength AF and minimum motion photometry have been found [39, 129], certain criticisms have been leveled at the one-wavelength method. Principally, the problem lies with the assumption that the fluorophores, i.e. lipofuscin, are distributed evenly across the imaging field. This is not the case [165] and, as a result, any attenuation in AF could be due to the presence of macular pigment but may also be a consequence of a lower level of lipofuscin in that area $[19,159,162]$. The use of a second, longer wavelength that is minimally absorbed by macular pigment eliminates this issue. Trieschmann et al. [162] compared the one and two-wavelength methods on 120 subjects. They concluded that onewavelength AF is acceptable as a screening method, particularly in view of its widespread availability in SLOs, whilst two-wavelength AF should always be used for precise MPOD assessment. The same conclusion was reached by Sharifzadeh et al. [153] using a CCD camera-based AF device.

\section{Assumptions}

Macular pigment assessment using AF assumes the following:

1. There are no fluorophores anterior to the macular pigment. Delori et al. [56] did find evidence of such a fluorophore and noted that it would cause a small underestimation of MPOD. This underestimation is minimized by detecting AF at a longer wavelength $-710 \mathrm{~nm}$ [56].

2. Lipofuscin at the fovea has the same excitation spectrum as lipofuscin in the surrounding retina. It is unknown whether this is entirely correct but according to Delori and colleagues [56], any differences are not big enough to affect the measured macular pigment spectral curve, as determined by their technique. The total amount of lipofuscin is known to vary across the retina but this is accounted for as long as the two-wavelength method is being used.

3. Any foveal-perifoveal differences in absorbers other than the macular pigment - retinal blood, photoreceptor pigments and RPE melanin - have a negligible effect on the measured MPOD. Delori et al. [56] investigated these assumptions in detail and found that retinal blood differences had virtually no effect and photoreceptor bleaching meant there was very little error in terms of photopigment differences. They did, however, find that RPE melanin slightly overestimated MPOD. 
4. Negligible macular pigment at any peripheral reference site. This is particularly important when the comparison technique is adopted but should be a true assumption because an adequate eccentricity is more easily accomplished with objective than subjective MPOD techniques.

\section{Validity}

The only study to date that has investigated the validity of AF in terms of a spectral comparison with in vitro MPOD data is that by Delori et al. [56]. Excitation wavelengths of 430, 470, 510 and $550 \mathrm{~nm}$ were used on 147 healthy-eyed subjects, plus 450, 490 and 530 $\mathrm{nm}$ on two of these subjects. The resultant spectral profiles for seven subjects are shown in Figure 12, along with the equivalent spectral profiles using a FR technique. The curves are in very good agreement with the chosen in vitro macular pigment curve, attesting to the validity of the AF method of MPOD measurement, in healthy eyes at least.

\section{INSERT FIGURE 12 ABOUT HERE}

Spatial profiles (or maps) of MPOD are the norm for AF imaging. In terms of validity, Robson et al. [39] demonstrated the symmetrical nature of macular pigment distribution (Figure 13), in line with findings using HFP and FR. Also like HFP and FR, however, an array of interindividual macular pigment distributions have been found. Figure 14, for instance, shows two distinct MPOD spatial profiles from a study by Wolf-Schnurrbusch et al. [154]. The top image is the classic distribution of a central peak in macular pigment with a rapid decline as distance from the fovea increases. The bottom image has a central peak in macular pigment followed by a decline, and there is also a secondary peak (a 'parafoveal ring') before further decline. In this particular study, the average eccentricity of the parafoveal ring was $0.66^{\circ}$ from the fovea, which is in line with several other studies [59, 116, 117]. Many researchers now propose that the total complement of macular pigment, rather than the peak amount, may better represent an individual's risk for, or protection from, AMD (see review by Bernstein et al. [155]).

\section{INSERT FIGURES 13 AND 14 ABOUT HERE}

In several studies, AF has been compared with other techniques of MPOD assessment, including HFP, using the same set of subjects $[39,56,60,129,141]$. All have shown good correlations, albeit with some systematic differences. 
$\underline{\text { Reliability }}$

As with FR, most tests of reliability concerning AF have been carried out within the same session and are perhaps, therefore, not as useful as inter-session reliability data.

Nevertheless, the results are impressive, indicating similar, if not better, reliability than HFP and FR. Table 4 contains a list of AF studies that have provided information on reliability.

\section{INSERT TABLE 4 ABOUT HERE}

\section{Advantages and disadvantages of $\mathrm{AF}$}

Advantages include: 1) its objectivity; as an objective test, AF requires no subject participation other than a short period of reasonable fixation 2) quick measurement time 3) spatial plots of macular pigment distribution produced as standard in AF imaging 4) good test-retest reliability 5) applicability to many subject populations, including children. Disadvantages include: 1) the need for pupil dilation 2) equipment expense 3) a lack of commercially-available two-wavelength SLOs 4) the need for photopigment bleaching and therefore unpleasant light levels 5) difficulty obtaining clear images from eyes with lens opacities.

\section{Resonance Raman spectroscopy}

With the exception of electrophysiology methods, resonance Raman spectroscopy (RRS) is the most recently developed MPOD technique and, arguably, the most controversial. First described by Bernstein et al. in 1998 [166], RRS takes advantage of lutein and zeaxanthin's ability to exhibit a phenomenon called Raman scattering [167]. Over the last ten years the use of RRS to measure MPOD has quickly gained momentum, with many papers published on its use $[57,111,168-178]$.

\section{Procedure}

When monochromatic light is directed at a molecule, some of the light is scattered. Most of the light is scattered elastically (Rayleigh scattering) but a small proportion is scattered inelastically (Raman scattering). When this inelastic back-scattering happens, there is a wavelength shift of the incident light, known as a Raman shift; the shift in wavelength is molecule-specific and therefore the back-scattered light can be collected and analyzed to identify the molecule in question. Usually the Raman signal is very weak and as such is not 
easily identified. However, if the incident wavelength overlaps with the absorption spectrum of the molecule, a large resonance enhancement of the Raman scattered light occurs and the molecule can be recognized. Carotenoids, including lutein and zeaxanthin, are an excellent example of this. When excited by $488 \mathrm{~nm}$ argon laser light, they exhibit a resonance enhancement of up to five orders of magnitude [169], with three characteristic Raman spectral peaks [166, 167, 171], as shown in Figure 15. The strongest peak is at 1525 $\mathrm{cm}^{-1}$ and this is the Raman line that is subsequently quantified in Raman counts (RCs).

RRS is completely different from almost all other MPOD techniques in that it measures absolute levels of macular pigment in a $1 \mathrm{~mm}\left(3.5^{\circ}\right)$ area, with no peripheral consideration at all. The researchers in this field claim that this is acceptable since the signal is derived directly from the pigment itself, rather than relying on light that must travel to deeper layers of the retina $[111,171]$ and that furthermore, the signal is only strong enough to register at carotenoid concentrations found in the macula, rather than in any other structures such as the cornea and lens [166].

\section{INSERT FIGURE 15 ABOUT HERE}

\section{$\underline{\text { Instrumentation }}$}

The setup for RRS detection of MPOD is shown in Figure 16. It consists of a $488 \mathrm{~nm}$ argon laser that is directed at the fovea. The returning back-scattered light is filtered so that only Raman shifted light is sent to the Raman spectrograph (via a fibre optic bundle). The spectrograph is linked to a CCD camera, which is in turn linked to a computer that is programmed to subtract the background fluorescence and quantify the intensity of the Raman peaks, in particular the $1525 \mathrm{~cm}^{-1}$ line. Optical alignment of the instrument to the fovea is achieved in human subjects by use of a red LED and a small portion of the blue argon laser light. The red LED is visible to the observer as a polka-dot pattern and the laser light as a blue disc. By small head movements along a head rest, the subject lines up the two images, at which point the operator pushes a button to begin the whole procedure. The $488 \mathrm{~nm}$ light is directed as a $0.5 \mathrm{~mW}, 1 \mathrm{~mm}$ spot onto the macula for 0.5 seconds [170, 171, 173]. Later studies using RRS have altered the settings slightly by using a $1 \mathrm{~mW}, 1 \mathrm{~mm}$ spot directed at the macula for 0.25 seconds $[57,174,175,179]$. Five measurements are taken at intervals of $30-180$ secs (the time is dependent on afterimage fading) and the original protocol [171] dictated that the three highest recordings are used in the data analysis, to allow for subjects that blink or misalign. 
Prior to measurement, pupil dilation to at least $6 \mathrm{~mm}$ is necessary [170, 171]; some later studies have found that dilation to at least $7 \mathrm{~mm}$ gives more reliable results $[57,174]$ - see discussion about pupil size in the 'validity' section.

\section{INSERT FIGURE 16 ABOUT HERE}

RESONANCE RAMAN IMAGING

One limitation of RRS in its classic form is that it is limited to measuring MPOD in a $1 \mathrm{~mm}$ $\left(3.5^{\circ}\right)$ area centred on the fovea, rather than being able to produce a spatial profile of macular pigment across a larger retinal area. The technique has therefore been extended into an imaging mode covering a $3.5 \mathrm{~mm}$ area; initially using excised donor eyes [172], then more recently, following further modification, using living volunteers $[175,178]$.

\section{Assumptions}

The assumptions associated with RRS are listed below. They are elaborated upon in the following section on validity.

1. Accurate alignment and fixation by the subject, and no significant head movement during image capture.

2. No significant effect on the Raman signal by differing levels of crystalline lens yellowing, i.e. absorption, within and across age groups.

3. No significant effect on the Raman signal by inter-individual differences in the level of lens diffusion (scatter and aberration).

\section{Validity}

The validity of RRS has been a subject of fierce debate in the literature [e.g. 54, 180, 181 186]. It is beyond the scope of this review to repeat the issues of contention verbatim; rather, the main points are summarized.

The research group that developed RRS for measurement of MPOD have shown that it very specific and sensitive to measurement of lutein and zeaxanthin [166, 170, 171]. However, unlike the other methods of MPOD measurement, RRS cannot generate spectral absorption curves of macular pigment to be compared with in vitro curves. Instead, the 
investigators attest to the validity of RRS in several other ways. Firstly, the strength of the Raman signal from donor maculae has been compared with actual macular pigment levels as measured by HPLC (Figure 17) [166]. The results revealed a highly linear correlation $(r=$ 0.94, no $p$-value provided). Secondly, a model eye containing known amounts of lutein was measured with the Raman device (Figure 18) and the results again demonstrated a linear correlation ( $r=0.99$, no $p$-value provided) [171]. Thirdly, the Raman signals from six intact monkey eyes were determined then compared with their HPLC-measured macular pigment level (Figure 19) [171]. The correlation between the two was good but not perfect $(r=0.68$, no $p$-value provided); the authors attributed this to differences in the detection area between the Raman (1 mm) and the HPLC (5 mm) method, and to difficulties with foveal alignment. Finally, Bernstein et al. [170] found a perfect linear correlation between Raman signals from lutein and zeaxanthin solutions placed in a model eye and their known concentrations (Figure 20A), up to about 0.35 density units (equivalent in this case to $1600 \mathrm{RCs}$ ). At higher concentrations, the Raman response became saturated and therefore non-linear (Figure 20B), as a result of the $488 \mathrm{~nm}$ laser beam being unable to penetrate increasingly dense carotenoid concentrations [170]. Ermakov et al. [174] found a similar linear then non-linear response for various zeaxanthin concentrations, again using a model eye.

\section{INSERT FIGURES 17-20 ABOUT HERE}

Those questioning the validity of RRS as a method of MPOD measurement have made several criticisms of the above calibration procedures. The issues of contention include whether or not the model eye is a true representation of a real in vivo eye; whether or not the increasing underestimation of MPOD at higher concentrations is a problem in ordinary subject populations; and the credibility of using the monkey data as evidence of validity, given that the level of RCs was in general far higher than most human levels and well above the point at which the plateau occurs in the external calibration curve (see Figures 19 and 20).

Studies of MPOD using RRS have consistently shown a strong decline in macular pigment with increasing age (see, for example, Figure 21). Hammond et al. [54] argue that this is another area where the validity of RRS is questionable, since with all other MPOD techniques, there appears to be little or no age-related macular pigment decline [141]. The developers of RRS believe that the decrease is genuine [110,111, 169-171, 173-175] and not just attributable to increasing lens absorption (yellowing) and diffusion (scatter and aberration) with age, as suggested by Hammond and Wooten [54, 182-184]. An independent study simulating incremental increases in lens yellowing and scatter found that the Raman 
signal intensity was significantly attenuated as the density of the yellow and scatter filters increased [179]. The authors concluded that when using RRS to assess MPOD, the status of the lens needs to be taken into account. In other words, the large decline in MPOD with age reported in many RRS studies is unlikely to represent a true drop in macular pigment levels and therefore the validity of RRS in older subjects, at least, is uncertain.

\section{INSERT FIGURE 21 ABOUT HERE}

Another reason for the decrease in MPOD with age - as found by RRS - may be due in part to inadequate pupil dilation. Studies have shown that the Raman signal is weakened when pupil diameter is smaller than $7 \mathrm{~mm}[57,171,174]$. This is because the entrance/exit pupil of the Raman instrument is also $7 \mathrm{~mm}$ and thus any pupil diameter less than this will result in a loss of signal [171, 174]. Neelam et al. [57] found that the significant age-related decline in RRS-derived MPOD of their subject population was reduced to a non-significant level when subjects with inadequate pupil dilation $(<7 \mathrm{~mm})$ were excluded. It would therefore seem that an inability to sufficiently dilate the pupils of some older individuals might contribute to the decline in MPOD seen with RRS. Further to this, small head movements in subjects whose pupil diameter is at the $7 \mathrm{~mm}$ limit could also reduce the Raman signal, regardless of age $[54,176,182-184,186]$, although Bernstein and colleagues may contend that their procedure of taking the three highest RCs of five measurements allows for such head movements.

Widely varying macular pigment spatial profiles have been observed using the RR imaging (RRI) device in living human subjects, including asymmetries and local depletions [178]. Although such distributions are not normally typical of other MPOD techniques, in comparing the integrated macular pigment densities of the entire imaged area with the densities measured over the same area with an AF method, the investigators found a very high correlation in 17 subjects ( $r=0.89$, no $p$-value provided). Furthermore, the integrated macular pigment levels of 11 donor maculae measured with the $R R I$ instrument were compared with the levels as measured by HPLC. The correlation between the two was very strong $(r=0.92, p<0.0001)$, although the influence of the ocular media was removed and therefore may have lead to an erroneously high agreement. Nevertheless, the validity of RRI looks promising.

Indirect methods of assessing validity, mentioned previously, consist of comparisons with more established, validated techniques, and the ability to show rises in MPOD with increased lutein and/or zeaxanthin intake. The latter has apparently been proven, although 
the study was only briefly described [110]. RRS-measured MPOD has been compared with HFP-measured MPOD in only two, detailed published papers [57, 176]. Neelam et al. [57], using Bland-Altman plots, demonstrated an agreement between the two techniques close to statistical significance. The correlation, as described by Pearson product-moment correlation coefficient, was $r=0.32$ (derived by Hammond and Wooten [184]). Although statistically significant, this correlation only explains $10 \%$ of the variance in the two methods [184]. Hogg et al. [176] also found a weak, albeit statistically significant, correlation between RRS and HFP ( $r=0.26, p=0.012)$. They did not feel it was good enough for the two techniques to be interchangeable. Bernstein et al. [110] reported a better correlation $(r=0.467, p=0.0024)$ in 40 healthy subjects, but their study was not described in any detail.

\section{$\underline{\text { Reliability }}$}

RRS appears to exhibit good within- and between-session reliability in the majority of studies (Table 5). The high variation in readings experienced in subjects from a study by Obana et al. [177], particularly in individuals with age-related maculopathy (ARM), is a notable exception. This is the only study that has provided any RRS reliability data for subjects with ocular disease and is an indication, perhaps, that more data is needed before evaluating information on MPOD in these populations. That said, 32 out of the 180 eyes with ARM had worse visual acuity than the recommended limit for RRS of 6/24 (20/80). This may have contributed to the variation. In view of the variation, Obana and colleagues chose to accept only the highest of the five Raman readings in all their subjects [177]. This means that $80 \%$ of the measurements were rejected, which seems a lot for a technique claiming to be validated. Nevertheless, many authorities have shown that MPOD measurements, as derived by RRS, do have good test-retest reliability (see Table 5).

\section{INSERT TABLE 5 ABOUT HERE}

\section{Advantages and disadvantages of RRS}

Advantages include: 1) sensitivity and specificity for retinal carotenoids 2) rapid measurements requiring only momentary fixation from the subject 3 ) the possibility of quickly generated, detailed spatial distribution plots of macular pigment, using the RRI method 4) reasonable reliability 5) measurements possible in many individuals, including those with reduced acuity, up to 6/24 (20/80). Disadvantages include: 1) the need for pupil dilation 2) the reliance on subjects for accurate alignment, i.e. the lack of an objective alignment procedure 3) afterimages between measurements 3) questionable validity 4) attenuation of 
the Raman signal with changes in the ocular media and inadequate pupil dilation 5) instrumentation that is highly specialized and expensive 6) RCs not being readily convertible to macular pigment density units, making direct comparisons with other techniques difficult.

\section{Electrophysiology - visual evoked potentials}

The first suggestion that visual evoked potentials (VEPs) could potentially be used to detect macular pigment was made over ten years ago, by Moreland et al. [37]. This was investigated further by Robson et al. [92] some time later. However, it is only very recently that this particular technique has looked like it could be a truly viable method for measuring MPOD. Using steady-state VEPs, Robson and Parry [41] measured MPOD across a range of eccentricities in three subjects. Blue-green gratings on a colour monitor were employed and these same gratings were also used to measure MPOD with HFP (see 'recent developments' in HFP section). The VEP and HFP results were compared with each other as well as with the equivalent MPOD as measured by minimum motion photometry. This required a correction factor on the part of the VEP and HFP results, to allow for the overlapping phosphor emissions of the blue and green stimuli. The correlation between all three techniques was excellent $(r \geq 0.94, p<0.0005$, in all cases), suggesting that steadystate VEPs have potential as a valid, objective method for measuring macular pigment and its distribution.

\section{DISCUSSION}

There are currently two main psychophysical techniques for measuring MPOD in vivo, and three main objective techniques. All take advantage of the spectral absorption properties of macular pigment but in very diverse ways. This diversity may be useful for macular pigment research but it does present difficulties for those wishing to compare MPOD values between techniques. For instance, does the value represent the peak density of macular pigment, the density of macular pigment at a certain point within the fovea, or the total amount within the target area?

If macular pigment measurement is to become commonplace in large populations, then equipment investors will have an important decision to make with regards to the method they choose to employ. Unfortunately, as each MPOD technique has its own benefits and limitations, there is no clear ideal choice, as highlighted by Beatty, van Kuijk and Chakravarthy [187]. Heterochromatic flicker photometry is probably the most affordable choice. It is also an established, valid and reliable method, particularly when protocols are 
followed as per 'customized' HFP [50, 52]. There is, however, the problem that some individuals find this task very difficult and their results cannot necessarily be relied upon. $A$ commercially available objective technique would therefore be desirable, possibly through adaptation of a scanning laser ophthalmoscope or fundus camera. The former is often used in a hospital setting and the latter is commonly found in optometric practice. A future objective technique could make use of FR or AF to assess MPOD, although AF may be preferred over FR because it is less influenced by light scatter and appears to have better reliability. Both have the facility to measure the spatial distribution of macular pigment and this seems to be an increasingly useful advantage $[8,39,74,108,116]$. Bhosale, Zhao and Bernstein [112] observed elevated lutein levels at the macula and at the peripheral retina in donors known to be using high dose lutein supplements. Therefore, if non-macula areas are not taken into account, the total complement of macular pigment may be underestimated, particularly with methods such as HFP, where the eccentric references are assumed to have virtually no macular pigment. The main issue associated with macular pigment screening using an objective technique is the need for pupil dilation, although several non-mydriatic devices have now been developed [58, 59, 153].

It is our view that the measurement of MPOD is best conducted using an objective technique based on FR or AF but we acknowledge that a commercial instrument capable of this is not currently available. The development of such an instrument will aid research in this area and provide a better understanding of the relationship between MPOD and AMD, as well as supporting MPOD screening in a clinical setting. 


\section{Figure and table legends}

Figure 1. Schematic diagram of a Maxwellian view optical system, as used by Wooten et al. [46]. A1-A3 = apertures 1-3. BF = blocking filter (removes stray light). BS1 and BS2 = beam splitters 1 and 2. $\mathrm{C}$ = flicker vanes with a first surface mirror (produces alternation of the test and reference lights). HM1-HM3 = hot mirrors 1-3 (reduce heat transfer). IF1 and IF2 = interference filters 1 and 2 . L1-L17 = lenses 1-17 (achromatic, planoconvex). $M=$ monochromator (produces the test wavelength). M1-M4 = mirrors 1-4 (right angle, first surface). ND = neutral density filter (together with interference filters, produces the reference and background wavelengths). $\mathrm{R}=$ reticle. $\mathrm{S}=$ source light, in this case a xenon arc lamp. $\mathrm{W}$ = wedge (used to adjust the radiance of the test light). [Reprinted from Wooten et al. [46], with permission from the Association for Research in Vision and Ophthalmology.]

Figure 2. Schematic diagram of a free view optical system, as used by Wooten et al. [46]. $\mathrm{A} 1$ and $\mathrm{A} 2=$ apertures 1 and $2 . \mathrm{BS}=$ beam splitter. D1 and D2 = optical diffusers (increase transmission efficiency). $\mathrm{H}$ = hole (1 inch circular viewing hole). $\mathrm{L} 1$ and $\mathrm{L} 2=$ lenses 1 and 2 (achromatic, planoconvex). PC = photocell. $S 1$ and $S 2=$ source lights 1 and $2(3 \times 470 \mathrm{~nm}$ LEDs for S1, i.e. background field, and $2 \times 458 \mathrm{~nm}$ plus $1 \times 570 \mathrm{~nm}$ for S2, i.e. test field). [Reprinted from Wooten et al. [46], with permission from the Association for Research in Vision and Ophthalmology.]

Figure 3. A detailed spatial profile of one subject's macular pigment density, obtained using heterochromatic flicker photometry. Here, measurements have been taken along the horizontal (filled squares) and vertical (open circles) meridians of the retina, demonstrating a rapid and symmetrical decline in macular pigment with increasing eccentricity [Reprinted from Hammond et al. [44], with permission from the Optical Society of America.]

Figure 4. The relative macular pigment spectral absorption profile of one subject (filled squares), as derived by heterochromatic flicker photometry. The continuous curve is an in vitro combination template as described in the text. Small deviations from the template at wavelengths below about $440 \mathrm{~nm}$ are typical. The reason for this is not clear and several theories have been proposed [see, for example, 54, 66, 71]. However, above $440 \mathrm{~nm}$, the in vivo and in vitro methods are in very close agreement, so measurements of peak macular pigment optical density should remain accurate. [Reprinted from Wooten and Hammond [71], with permission from the American Academy of Optometry.] 
Figure 5. A high correlation $(r=0.91, p<0.00001)$ between macular pigment optical density measured with a centrally positioned $1^{\circ}$ test stimulus and a $12^{\prime}$ test stimulus positioned $0.5^{\circ}$ from the central fovea lends support to the edge hypothesis of heterochromatic flicker photometry. [Reprinted from Hammond et al. [44], with permission from the Optical Society of America.]

Figure 6. Left panel: Macular pigment distribution in the central $1.5^{\circ}$, as determined by Bone et al. [68], using heterochromatic flicker photometry. The horizontal line is the average macular pigment optical density (MPOD) for 10 subjects, calculated using a centrally positioned $1.5^{\circ}$ circular test stimulus (grey area $= \pm 1$ standard deviation). The filled circles depict the average MPOD at various eccentricities (bars $= \pm 1$ standard deviation), determined using a number of annular stimuli with central fixation marks (right panel). Whereas Hammond et al. [44] used a very small stimulus placed at the required retinal eccentricity, Bone and colleagues have used annular stimuli. This is an alternative method of knowingly measuring MPOD at a retinal eccentricity equivalent to the stimuli radii. The intersection point at $0.38^{\circ}$ is the position at which MPOD appears to be measured when using a $1.5^{\circ}$ test stimulus, i.e. $51 \%$ of the stimulus radius. Right panel: The four annular stimuli and two circular stimuli (1.17 ${ }^{\circ}$ circular stimulus not used in this graph). ID $=$ inner diameter, OD = outer diameter. [Reprinted from Bone et al. [68], with permission from Elsevier.]

Figure 7. Test configuration used in minimum motion photometry (left panel), with the associated stimuli dimensions given in the table (right panel). [Reprinted from Robson et al. [129], with permission from Pion Limited.]

Figure 8. Apparent motion photometry. Left panel: Apparent movement to the right when the red bars are brighter than the blue, and vice-versa. Right panel: The test configuration for a parafoveal target [130].

Figure 9. Schematic diagram of the Foveal Reflection Analyzer 1, from Zagers et al. [146]. In (a): $F=$ spectral filters. Lamp $=30 \mathrm{~W}$ halogen lamp. $L 1-L 11=$ lenses $1-11 . L f=$ front lens. $\mathrm{Li}=$ insertable lens. $\mathrm{Mh}=$ mirror with central hole. $\mathrm{Mi}=$ insertable mirror. $\mathrm{P}=$ pupil plane. $\mathrm{R}=$ retinal plane. $\mathrm{P}^{\prime}$ and $\mathrm{R}^{\prime}$ = planes conjugate to $\mathrm{P}$ and $\mathrm{R}$. V = video camera. $\ln (\mathrm{b})$ : The dilated pupil with entrance and exit pupil shown to scale (left panel). The illuminated field and the concentric sampled field, with fixation cross hairs (right panel). [Reprinted from Zagers et al. [146], with permission from the Optical Society of America.] 
Figure 10. Schematic diagram of the Macular Pigment Reflectometer, from van de Kraats et al. [58]. L1-L8 = lenses 1-8. M = mirror. [Reprinted from van de Kraats et al. [58], with permission from SPIE.]

Figure 11. Spatial profiles of macular pigment density in three age groups, obtained by fundus reflectometry: (a) young (24.8 \pm 2.6 years), (b) mid-age (40.2 \pm 8.3 ) and (c) old (67.5 \pm 7.1). [Reprinted from Chen et al. [108], with permission from Informa Healthcare.]

Figure 12. Left panel: Log ratio autofluorescence (AF) plotted against wavelength for seven subjects (symbols) along with the scaled macular pigment spectra (curves). The age of each subject is given to the left of each curve and the derived macular pigment optical density (with $r^{2}$ values of the fits) is given to the right. Right panel: The equivalent fundus reflectometry ('RE') results. [Reprinted from Delori et al. [56], with permission from the Optical Society of America.]

Figure 13. Autofluorescence images (first and third columns) and macular pigment spatial profiles (second and fourth columns) for 8 subjects $(A-H)$. Open circles indicate the vertical meridian and filled circles the horizontal meridian. The arrows indicate disruptions due to prominent blood vessels. [Reprinted from Robson et al. [39], with permission from Elsevier.]

Figure 14. Two example macular pigment spatial profiles with their corresponding autofluorescence images: (a) classic profile - central peak in macular pigment followed by a rapid decline, and (b) parafoveal ring profile - central peak in macular pigment, plus a secondary peak, before further decline. [Reprinted from Wolf-Schnurrbusch et al. [154], with permission from the Association for Research in Vision and Ophthalmology.]

Figure 15. In vivo Raman spectra of a healthy subject, showing the characteristic peaks of macular pigment. Top trace = before subtraction of the background ocular fluorescence. Bottom trace $=$ after subtraction. [Reprinted from Gellermann et al. [171], with permission from the Optical Society of America.]

Figure 16. Schematic diagram of a resonance Raman spectroscopy macular pigment detector. $\mathrm{Ar}^{+}$laser $=$air-cooled argon laser. $\mathrm{BS}=$ dichroic beam splitter. $\mathrm{F}=$ filter. $\mathrm{LED}=$ light emitting diode. $\mathrm{L} 1-4$ = lenses $1-4 . \mathrm{M}=$ Mirror. NF = holographic rejection notch filter. $\mathrm{TB}=$ trigger button. VHTF = volume holographic transmission grating. [Reprinted from Gellermann et al. [171], with permission from the Optical Society of America.] 
Figure 17. Macular pigment measurements in seven human donor maculae, measured by high-performance liquid chromatography and resonance Raman spectroscopy. [Reprinted from Bernstein et al. [166], with permission from the Association for Research in Vision and Ophthalmology.]

Figure 18. Macular pigment measurements by resonance Raman spectroscopy of four known lutein concentrations placed in a model eye. [Reprinted from Gellermann et al. [171], with permission from the Optical Society of America.]

Figure 19. Macular pigment measurements in six monkey eyes, measured by highperformance liquid chromatography and resonance Raman spectroscopy. [Reprinted from Gellermann et al. [171], with permission from the Optical Society of America.]

Figure 20. Macular pigment measurements by resonance Raman spectroscopy of known lutein and zeaxanthin concentrations placed in a model eye: $A$ = linear response range (filled circles are lutein, open circles are zeaxanthin). $B=$ linear and non-linear response range. [Reprinted from Bernstein et al. [170], with permission from Elsevier.]

Figure 21. The decline in macular pigment optical density with age, as measured by resonance Raman spectroscopy. [Reprinted from Bernstein et al. [170], with permission from Elsevier.]

Table 1. A summary of the main differences between Maxwellian and free view optical systems.

Table 2. A list of all heterochromatic flicker photometry (HFP) studies to date that have given statistical data on test-retest reliability.

a Snodderly et al. [50] also calculated the equivalent values for the left eye, which were $r=$ $0.86,22 \%$ test-retest difference and a coefficient of repeatability of 0.21 .

$\mathrm{ARM}=$ age-related maculopathy, the general term for degeneration of the macular region of the retina, which is normally separated into the two categories of early and late ARM, with the latter also being called age-related macular degeneration [188].

MPOD = macular pigment optical density.

$\mathrm{RE}=$ right eye.

$\mathrm{RPE}=$ retinal pigment epithelium. 
Table 3. A list of all fundus reflectometry (FR) studies to date that have given statistical data on reliability.

$\mathrm{ARM}=$ age-related maculopathy.

FRA = Foveal Reflection Analyzer, a purpose-built reflectometer.

MPR = Macular Pigment Reflectometer, a purpose-built reflectometer.

SLO = scanning laser ophthalmoscope.

Table 4. A list of all autofluorescence (AF) studies to date that have given statistical data on reliability.

AMD = age-related macular degeneration .

MPOD = macular pigment optical density.

Table 5. A list of all resonance Raman spectroscopy (RRS) studies to date that have given statistical data on reliability.

$\mathrm{ARM}=$ age-related maculopathy, the general term for degeneration of the macular region of the retina, which is normally separated into the two categories of early and late ARM, with the latter also being called age-related macular degeneration (AMD) [188].

MPOD = macular pigment optical density 


\section{REFERENCES}

1. Bone RA, Landrum JT, Hime GW, Cains A, Zamor J (1993) Stereochemistry of the human macular carotenoids. Invest Ophthalmol Vis Sci 34: 2033-2040

2. Malinow MR, Feeneyburns L, Peterson LH, Klein ML, Neuringer M (1980) Diet-related macular anomalies in monkeys. Invest Ophthalmol Vis Sci 19: 857-863

3. Neuringer M, Sandstrom MM, Johnson EJ, Snodderly DM (2004) Nutritional manipulation of primate retinas, I: effects of lutein or zeaxanthin supplements on serum and macular pigment in xanthophyll-free rhesus monkeys. Invest Ophthalmol Vis Sci 45: 3234-3243 4. Sommerburg O, Keunen JEE, Bird AC, van Kuijk F (1998) Fruits and vegetables that are sources for lutein and zeaxanthin: the macular pigment in human eyes. Br J Ophthalmol 82: 907-910

5. Snodderly DM, Auran JD, Delori FC (1984) The macular pigment .II. Spatial distribution in primate retinas. Invest Ophthalmol Vis Sci 25: 674-685

6. Snodderly DM, Brown PK, Delori FC, Auran JD (1984) The macular pigment .I. Absorbance spectra, localization, and discrimination from other yellow pigments in primate retinas. Invest Ophthalmol Vis Sci 25: 660-673

7. Bone RA, Landrum JT, Fernandez L, Tarsis SL (1988) Analysis of the macular pigment by HPLC - retinal distribution and age study. Invest Ophthalmol Vis Sci 29: 843-849

8. Trieschmann M, van Kuijk F, Alexander R, Hermans $P$, Luthert $P$, Bird AC, Pauleikhoff D (2008) Macular pigment in the human retina: histological evaluation of localization and distribution. Eye 22: 132-137

9. Bone RA, Landrum JT, Cains A (1992) Optical density spectra of the macular pigment in vivo and in vitro. Vision Res 32: 105-110

10. Ham WT, Mueller HA, Sliney DH (1976) Retinal sensitivity to damage from short wavelength light. Nature 260: 153-155

11. Hammond BR, Caruso-Avery M (2000) Macular pigment optical density in a southwestern sample. Invest Ophthalmol Vis Sci 41: 1492-1497

12. Landrum JT, Bone RA (2001) Lutein, zeaxanthin, and the macular pigment. Arch Biochem Biophys 385: 28-40

13. Khachik F, Bernstein PS, Garland DL (1997) Identification of lutein and zeaxanthin oxidation products in human and monkey retinas. Invest Ophthalmol Vis Sci 38: 1802-1811 14. Leibowitz HM, Krueger DE, Maunder LR, Milton RC, Kini MM, Kahn HA, Nickerson RJ, Pool J, Colton TL, Ganley JP, Loewenstein JI, Dawber TR (1980) The Framingham Eye Study monograph: an ophthalmological and epidemiological study of cataract, glaucoma, diabetic retinopathy, macular degeneration, and visual acuity in a general population of 2631 adults, 1973-1975. Surv Ophthalmol 24: 335-610

15. Klein R, Klein BEK, Linton KLP (1992) Prevalence of age-related maculopathy - the Beaver Dam Eye Study. Ophthalmology 99: 933-943

16. Mitchell P, Smith W, Attebo K, Wang JJ (1995) Prevalence of age-related maculopathy in Australia - the Blue Mountains Eye Study. Ophthalmology 102: 1450-1460

17. Beatty S, Murray IJ, Henson DB, Carden D, Koh HH, Boulton ME (2001) Macular pigment and risk for age-related macular degeneration in subjects from a Northern European population. Invest Ophthalmol Vis Sci 42: 439-446

18. Bone RA, Landrum JT, Mayne ST, Gomez CM, Tibor SE, Twaroska EE (2001) Macular pigment in donor eyes with and without AMD: A case-control study. Invest Ophthalmol Vis Sci 42: $235-240$

19. Trieschmann M, Spital G, Lommatzsch A, van Kuijk E, Fitzke F, Bird AC, Pauleikhoff D (2003) Macular pigment: quantitative analysis on autofluorescence images. Graefes Arch Clin Exp Ophthalmol 241: 1006-1012

20. Ciulla TA, Hammond BR (2004) Macular pigment density and aging, assessed in the normal elderly and those with cataracts and age-related macular degeneration. Am J Ophthalmol 138: 582-587 
21. LaRowe TL, Mares JA, Snodderly DM, Klein ML, Wooten BR, Chappell R, Grp CMPS (2008) Macular pigment density and age-related maculopathy in the carotenoids in agerelated eye disease study - an ancillary study of the women's health initiative.

Ophthalmology 115: 876-883

22. Bone RA, Landrum JT, Tarsis SL (1985) Preliminary identification of the human macular pigment. Vision Res 25: 1531-1535

23. Handelman GJ, Dratz EA, Reay CC, Vankuijk F (1988) Carotenoids in the human macula and whole retina. Invest Ophthalmol Vis Sci 29: 850-855

24. Handelman GJ, Snodderly DM, Krinsky NI, Russett MD, Adler AJ (1991) Biological control of primate macular pigment - biochemical and densitometric studies. Invest Ophthalmol Vis Sci 32: 257-267

25. Wüstemeyer H, Moessner A, Jahn C, Wolf S (2003) Macular pigment density in healthy subjects quantified with a modified confocal scanning laser ophthalmoscope. Graefes Arch Clin Exp Ophthalmol 241: 647-651

26. Trieschmann M, Beatty S, Nolan JM, Hense HW, Heimes B, Austermann U, Fobker M, Pauleikhoff D (2007) Changes in macular pigment optical density and serum concentrations of its constituent carotenoids following supplemental lutein and zeaxanthin: the LUNA study. Exp Eye Res 84: 718-728

27. Brown PK, Wald G (1963) Visual pigments in human and monkey retinas. Nature 200: 37-43

28. Bone RA, Sparrock JM (1971) Comparison of macular pigment densities in human eyes. Vision Res 11: 1057-1064

29. Stabell U, Stabell B (1980) Variation in density of macular pigmentation and in shortwave cone sensitivity with eccentricity. J Opt Soc Am 70: 706-711

30. Pease PL, Adams AJ (1983) Macular pigment difference spectrum from sensitivity measures of a single cone mechanism. Am J Optom Physiol Opt 60: 667-672

31. Pease PL, Adams AJ, Nuccio E (1987) Optical density of human macular pigment. Vision Res 27: 705-710

32. Ruddock KH (1963) Evidence for macular pigmentation from colour matching data.

Vision Res 61: 417-429

33. Moreland JD, Bhatt P (1984) Retinal distribution of macular pigment. Doc Ophthalmol Proc Ser 39: 127-132

34. Moreland JD, Alexander EC (1997) Effect of macular pigment on colour matching with field sizes in the $1^{\circ}$ to $10^{\circ}$ range. Doc Ophthalmol Proc Ser 59: 363-368

35. Davies NP, Morland AB (2002) Color matching in diabetes: optical density of the crystalline lens and macular pigments. Invest Ophthalmol Vis Sci 43: 281-289

36. Bone RA (1980) The role of the macular pigment in the detection of polarized light. Vision Res 20: 213-220

37. Moreland JD, Robson AG, Soto-Leon N, Kulikowski JJ (1998) Macular pigment and the colour-specificity of visual evoked potentials. Vision Res 38: 3241-3245

38. Moreland JD, Robson AG, Kulikowski JJ (2001) Macular pigment assessment using a colour monitor. Color Res Appl 26: S261-S263

39. Robson AG, Moreland JD, Pauleikhoff D, Morrissey T, Holder GE, Fitzke FW, Bird AC, van Kuijk $F$ (2003) Macular pigment density and distribution: comparison of fundus autofluorescence with minimum motion photometry. Vision Res 43: 1765-1775

40. Moreland JD (2004) Macular pigment assessment by motion photometry. Arch Biochem Biophys 430: 143-148

41. Robson AG, Parry NRA (2008) Measurement of macular pigment optical density and distribution using the steady-state visual evoked potential. Vis Neurosci 25: 575-583 42. Werner JS, Donnelly SK, Kliegl R (1987) Aging and human macular pigment density. Appended with translations from the work of MaxSchultze and Ewald Hering. Vision Res 27: 257-268

43. Hammond BR, Fuld K (1992) Interocular differences in macular pigment density. Invest Ophthalmol Vis Sci 33: 350-355 
44. Hammond BR, Wooten BR, Snodderly DM (1997) Individual variations in the spatial profile of human macular pigment. J Opt Soc Am A Opt Image Sci Vis 14: 1187-1196 45. Landrum JT, Bone RA, Joa H, Kilburn MD, Moore LL, Sprague KE (1997) A one year study of the macular pigment: the effect of 140 days of a lutein supplement. Exp Eye Res 65: $57-62$

46. Wooten BR, Hammond BR, Land RI, Snodderly DM (1999) A practical method for measuring macular pigment optical density. Invest Ophthalmol Vis Sci 40: 2481-2489 47. Beatty S, Koh HH, Carden D, Murray IJ (2000) Macular pigment optical density measurement: a novel compact instrument. Ophthalmic Physiol Opt 20: 105-111 48. Mellerio J, Ahmadi-Lari S, van Kuijk F, Pauleikhoff D, Bird A, Marshall J (2002) A portable instrument for measuring macular pigment with central fixation. Curr Eye Res 25: 37-47

49. Bone RA, Landrum JT (2004) Heterochromatic flicker photometry. Arch Biochem Biophys 430: 137-142

50. Snodderly DM, Mares JA, Wooten BR, Oxton L, Gruber M, Ficek T (2004) Macular pigment measurement by heterochromatic flicker photometry in older subjects: the carotenoids and age-related eye disease study. Invest Ophthalmol Vis Sci 45: 531-538 51. lannaccone A, Mura M, Gallaher KT, Johnson EJ, Todd WA, Kenyon E, Harris TL, Harris T, Satterfield S, Johnson KC, Kritchevsky SB, Study HA (2007) Macular pigment optical density in the elderly: findings in a large biracial midsouth population sample. Invest Ophthalmol Vis Sci 48: 1458-1465

52. Stringham JM, Hammond BR, Nolan JM, Wooten BR, Mammen A, Smollon W, Snodderly DM (2008) The utility of using customized heterochromatic flicker photometry (cHFP) to measure macular pigment in patients with age-related macular degeneration. Exp Eye Res 87: 445-453

53. van der Veen RL, Berendschot TT, Hendrikse F, Carden D, Makridaki M, Murray IJ (2009) A new desktop instrument for measuring macular pigment optical density based on a novel technique for setting flicker thresholds. Ophthalmic Physiol Opt 29: 127-137

54. Hammond BR, Wooten BR, Smollon B (2005) Assessment of the validity of in vivo methods of measuring human macular pigment optical density. Optom Vis Sci 82: 387-404 55. Viner C (2003) Measuring macular pigment levels: an in-practice procedure? Optician 226: $24-26$

56. Delori FC, Goger DG, Hammond BR, Snodderly DM, Burns SA (2001) Macular pigment density measured by autofluorescence spectrometry: comparison with reflectometry and heterochromatic flicker photometry. J Opt Soc Am A Opt Image Sci Vis 18: 1212-1230 57. Neelam K, O'Gorman N, Nolan J, O'Donovan O, Wong HB, Eong KGA, Beatty S (2005) Measurement of macular pigment: Raman spectroscopy versus heterochromatic flicker photometry. Invest Ophthalmol Vis Sci 46: 1023-1032

58. van de Kraats J, Berendschot T, Valen S, van Norren D (2006) Fast assessment of the central macular pigment density with natural pupil using the macular pigment reflectometer. J Biomed Opt 11: 064031

59. Bone RA, Brener B, Gibert JC (2007) Macular pigment, photopigments, and melanin: distributions in young subjects determined by four-wavelength reflectometry. Vision Res 47: 3259-3268

60. Canovas R, Lima VC, Garcia P, Morini C, Prata TS, Rosen RB (2010) Comparison between macular pigment optical density measurements using two-wavelength autofluorescence and heterochromatic flicker photometry techniques. Invest Ophthalmol Vis Sci 51: 3152-3156

61. Werner JS, Wooten BR (1979) Opponent chromatic response functions for an average observer. Percept Psychophys 25: 371-374

62. Tang CY, Yip HS, Poon MY, Yau WL, Yap MKH (2004) Macular pigment optical density in young Chinese adults. Ophthalmic Physiol Opt 24: 586-593

63. Loane E, Stack J, Beatty S, Nolan JM (2007) Measurement of macular pigment optical density using two different heterochromatic flicker photometers. Curr Eye Res 32: 555-564 
64. Kirby ML, Galea M, Loane E, Stack J, Beatty S, Nolan JM (2009) Foveal anatomic associations with the secondary peak and the slope of the macular pigment spatial profile. Invest Ophthalmol Vis Sci 50: 1383-1391

65. Snodderly DM, Hammond BR (1999) In vivo psychophysical assessment of nutritional and environmental influences on human ocular tissues: lens and macular pigment. In: Taylor A (eds) Nutritional and Environmental Influences on the Eye. CRC Press, Boca Raton, FL, pp 251-273

66. Werner JS, Bieber ML, Schefrin BE (2000) Senescence of foveal and parafoveal cone sensitivities and their relations to macular pigment density. J Opt Soc Am A Opt Image Sci Vis 17: 1918-1932

67. Hammond BR, Johnson EJ, Russell RM, Krinsky NI, Yeum KJ, Edwards RB, Snodderly DM (1997) Dietary modification of human macular pigment density. Invest Ophthalmol Vis Sci 38: 1795-1801

68. Bone RA, Landrum JT, Gibert JC (2004) Macular pigment and the edge hypothesis of flicker photometry. Vision Res 44: 3045-3051

69. Burke JD, Curran-Celentano J, Wenzel AJ (2005) Diet and serum carotenoid concentrations affect macular pigment optical density in adults 45 years and older. $\mathrm{J}$ Nutr 135: $1208-1214$

70. Lam RF, Rao SK, Fan DSP, Lau FTC, Lam DSC (2005) Macular pigment optical density in a Chinese sample. Curr Eye Res 30: 799-805

71. Wooten BR, Hammond BR (2005) Spectral absorbance and spatial distribution of macular pigment using heterochromatic flicker photometry. Optom Vis Sci 82: 378-386 72. Stringham JM, Hammond BR (2007) The glare hypothesis of macular pigment function. Optom Vis Sci 84: 859-864

73. Wenzel AJ, Sheehan JP, Burke JD, Lefsrud MG, Curran-Celentano J (2007) Dietary intake and serum concentrations of lutein and zeaxanthin, but not macular pigment optical density, are related in spouses. Nutr Res 27: 462-469

74. Wenzel AJ, Sheehan JP, Gerweck C, Stringham JM, Fuld K, Curran-Celentano J (2007) Macular pigment optical density at four retinal loci during 120 days of lutein supplementation. Ophthalmic Physiol Opt 27: 329-335

75. Nolan JM, Stringham JM, Beatty S, Snodderly DM (2008) Spatial profile of macular pigment and its relationship to foveal architecture. Invest Ophthalmol Vis Sci 49: 2134-2142 76. Stringham JM, Hammond BR (2008) Macular pigment and visual performance under glare conditions. Optom Vis Sci 85: 82-88

77. Stringham JM, Hammond BR, Wooten BR, Snodderly DM (2006) Compensation for light loss resulting from filtering by macular pigment: relation to the S-cone pathway. Optom Vis Sci 83: 887-894

78. Connolly EE, Beatty S, Thurnham DI, Loughman J, Howard AN, Stack J, Nolan JM (2010) Augmentation of macular pigment following supplementation with all three macular carotenoids: an exploratory study. Curr Eye Res 35: 335-351

79. Loane E, McKay GJ, Nolan JM, Beatty S (2010) Apolipoprotein E genotype is associated with macular pigment optical density. Invest Ophthalmol Vis Sci 51: 2636-2643

80. Ciulla TA, Curran-Celantano J, Cooper DA, Hammond BR, Danis RP, Pratt LM, Riccardi KA, Filloon TG (2001) Macular pigment optical density in a midwestern sample.

Ophthalmology 108: 730-737

81. Ciulla TA, Hammond BR, Yung CW, Pratt LM (2001) Macular pigment optical density

before and after cataract extraction. Invest Ophthalmol Vis Sci 42: 1338-1341

82. Hammond BR, Fuld K, Curran-Celentano J (1995) Macular pigment density in monozygotic twins. Invest Ophthalmol Vis Sci 36: 2531-2541

83. Nolan JM, Stack J, Donovan OO, Loane E, Beatty S (2007) Risk factors for age-related maculopathy are associated with a relative lack of macular pigment. Exp Eye Res 84: 61-74 84. Nolan JM, Stack J, O'Connell E, Beatty S (2007) The relationships between macular pigment optical density and its constituent carotenoids in diet and serum. Invest Ophthalmol Vis Sci 48: 571-582 
85. Schalch W, Cohn W, Barker FM, Kopcke W, Mellerio J, Bird AC, Robson AG, Fitzke FF, van Kuijk FJGM (2007) Xanthophyll accumulation in the human retina during supplementation with lutein or zeaxanthin - the LUXEA (LUtein Xanthophyll Eye

Accumulation) study. Arch Biochem Biophys 458: 128-135

86. Hammond BR, Wooten BR (2005) CFF thresholds: relation to macular pigment optical density. Ophthalmic Physiol Opt 25: 315-319

87. Cooper DA, Curran-Celentano J, Ciulla TA, Hammond BR, Danis RB, Pratt LM, Riccardi $\mathrm{KA}$, Filloon TG (2000) Olestra consumption is not associated with macular pigment optical density in a cross-sectional volunteer sample in Indianapolis. J Nutr 130: 642-647

88. Curran-Celentano J, Hammond BR, Ciulla TA, Cooper DA, Pratt LM, Danis RB (2001)

Relation between dietary intake, serum concentrations, and retinal concentrations of lutein and zeaxanthin in adults in a Midwest population. Am J Clin Nutr 74: 796-802

89. Bone RA, Landrum JT, Guerra LH, Ruiz CA (2003) Lutein and zeaxanthin dietary supplements raise macular pigment density and serum concentrations of these carotenoids in humans. J Nutr 992-998

90. Bone RA, Landrum JT, Cao Y, Howard AN, Alvarez-Calderon F (2007) Macular pigment response to a supplement containing meso-zeaxanthin, lutein and zeaxanthin. Nutr Metab 4: 12

91. Koh HH, Murray IJ, Nolan D, Carden D, Feather J, Beatty S (2004) Plasma and macular response to lutein supplement in subjects with and without age-related maculopathy: a pilot study. Exp Eye Res 79: 21-27

92. Robson AG, Holder GE, Moreland JD, Kulikowski JJ (2006) Chromatic VEP assessment of human macular pigment: comparison with minimum motion and minimum flicker profiles. Vis Neurosci 23: 275-283

93. Engles M, Wooten B, Hammond B (2007) Macular pigment: a test of the acuity hypothesis. Invest Ophthalmol Vis Sci 48: 2922-2931

94. Rodriguez-Carmona M, Kvansakul J, Harlow JA, Kopcke W, Schalch W, Barbur JL (2006) The effects of supplementation with lutein and/or zeaxanthin on human macular pigment density and colour vision. Ophthalmic Physiol Opt 26: 137-147

95. Kvansakul J, Rodriguez-Carmona M, Edgar DF, Barker FM, Kopcke W, Schalch W, Barbur JL (2006) Supplementation with the carotenoids lutein or zeaxanthin improves human visual performance. Ophthalmic Physiol Opt 26: 362-371

96. Makridaki M, Carden D, Murray IJ (2009) Macular pigment measurement in clinics: controlling the effect of the ageing media. Ophthalmic Physiol Opt 29: 338-344

97. Nolan JM, Kenny R, O'Regan C, Cronin H, Loughman J, Connolly EE, Kearney P, Loane E, Beatty S (2010) Macular Pigment Optical Density in an Ageing Irish Population: The Irish Longitudinal Study on Ageing. Ophthalmic Res 44: 131-139

98. Wüstemeyer H, Jahn C, Nestler A, Barth T, Wolf S (2002) A new instrument for the quantification of macular pigment density: first results in patients with AMD and healthy subjects. Graefes Arch Clin Exp Ophthalmol 240: 666-671

99. Curcio CA, Allen KA, Sloan KR, Lerea CL, Hurley JB, Klock IB, Milam AH (1991)

Distribution and morphology of human cone photoreceptors stained with anti-blue opsin. $\mathrm{J}$

Comp Neurol 312: 610-624

100. Curcio CA, Sloan KR (1992) Packing geometry of human cone photoreceptors variation with eccentricity and evidence for local anisotropy. Vis Neurosci 9: 169-180 101. Curcio CA, Sloan KR, Kalina RE, Hendrickson AE (1990) Human photoreceptor topography. J Comp Neurol 292: 497-523

102. Cicerone CM, Nerger JL (1989) The relative numbers of long-wavelength-sensitive to middle-wavelength-sensitive cones in the human fovea centralis. Vision Res 29: 115-128 103. Nerger JL, Cicerone CM (1992) The ratio of L-cones to M-cones in the human parafoveal retina. Vision Res 32: 879-888

104. Knau H, Jagle H, Sharpe LT (2001) L/M cone ratios as a function of retinal eccentricity. Color Res Appl 26: S128-S132 
105. Hammond BR, Wooten BR, Snodderly DM (1998) Preservation of visual sensitivity of older subjects: association with macular pigment density. Invest Ophthalmol Vis Sci 39: 397406

106. Brindley GS, Du Croz JJ, Rushton WA (1966) The flicker fusion frequency of the bluesensitive mechanism of colour vision. J Physiol 183: 497-500

107. Nolan J, O'Donovan O, Kavanagh H, Stack J, Harrison M, Muldoon A, Mellerio J, Beatty S (2004) Macular pigment and percentage of body fat. Invest Ophthalmol Vis Sci 45: 3940-3950

108. Chen SF, Chang Y, Wu JC (2001) The spatial distribution of macular pigment in humans. Curr Eye Res 23: 422-434

109. Berendschot T, van Norren D (2004) Objective determination of the macular pigment optical density using fundus reflectance spectroscopy. Arch Biochem Biophys 430: 149-155 110. Bernstein PS, Zhao DY, Sharifzadeh M, Ermakov IV, Gellermann W (2004) Resonance Raman measurement of macular carotenoids in the living human eye. Arch Biochem Biophys 430: 163-169

111. Gellermann W, Bernstein PS (2004) Noninvasive detection of macular pigments in the human eye. J Biomed Opt 9: 75-85

112. Bhosale P, Zhao DY, Bernstein PS (2007) HPLC measurement of ocular carotenoid levels in human donor eyes in the lutein supplementation era. Invest Ophthalmol Vis Sci 48: 543-549

113. Hammond BR, Ciulla TA, Snodderly DM (2002) Macular pigment density is reduced in obese subjects. Invest Ophthalmol Vis Sci 43: 47-50

114. Gallaher KT, Mura M, Todd WA, Harris TL, Kenyon E, Harris T, Johnson KC, Satterfield S, Kritchevsky SB, lannaccone A, HIth ABCS (2007) Estimation of macular pigment optical density in the elderly: test-retest variability and effect of optical blur in pseudophakic subjects. Vision Res 47: 1253-1259

115. Snodderly DM, Handelman GJ, Adler AJ (1991) Distribution of individual macular pigment carotenoids in central retina of macaque and squirrel-monkeys. Invest Ophthalmol Vis Sci 32: 268-279

116. Berendschot T, van Norren D (2006) Macular pigment shows ringlike structures. Invest Ophthalmol Vis Sci 47: 709-714

117. Delori FC, Goger DG, Keilhauer C, Salvetti P, Staurenghi G (2006) Bimodal spatial distribution of macular pigment: evidence of a gender relationship. J Opt Soc Am A Opt Image Sci Vis 23: 521-538

118. Wyszecki G, Stiles WS (1982) The Eye. In: (eds) Color Science: Concepts and Methods, Quantitative Data and Formulae. Wiley-Interscience, New York, pp 112-114 119. Bartlett H, Acton J, Eperjesi F (2010) Clinical evaluation of the MacuScope macular pigment densitometer. Br J Ophthalmol 94: 328-331

120. Hagen S, Krebs I, Glittenberg C, Binder S (2010) Repeated measures of macular pigment optical density to test reproducibility of heterochromatic flicker photometry. Acta Ophthalmol 88: 207-211

121. van der Veen RLP, Berendschot T, Makridaki M, Hendrikse F, Carden D, Murray IJ (2009) Correspondence between retinal reflectometry and a flicker-based technique in the measurement of macular pigment spatial profiles. J Biomed Opt 14: 064046

122. Johnson EJ, Chung HY, Caldarella SM, Snodderly DM (2008) The influence of supplemental lutein and docosahexaenoic acid on serum, lipoproteins, and macular pigmentation. Am J Clin Nutr 87: 1521-1529

123. Troxler DIPV (1804) Über das Verschwinden gegebener Gegenstände innerhalb unseres Gesichtskreises. In: Himly J, Schmidt JA (eds) Ophthalmologische Bibliothek. Jena: Fromann, pp 1-53

124. Stumpf $P$ (1911) On the dependence of the visual sensation of movement and its negative aftereffect on the stimulation processes on the retina. Z Psychol 59: 321-330 125. Todorović D (1996) A gem from the past: Pleikart Stumpf's (1911) anticipation of the aperture problem, Reichardt detectors, and perceived motion loss at equiluminance.

Perception 25: 1235-1242 
126. Moreland JD (1980) A modified Moreland anomaloscope using optokinetic nystagmus to define colour matches objectively. In: Verriest G (eds) Colour Vision Deficiencies V.

Hilger, Bristol, pp 189-191

127. Moreland JD (1982) Spectral sensitivity measurements by motion photometry. Doc Ophthalmol Proc Ser 33: 61-66

128. Anstis SM, Cavanagh $P$ (1983) A minimum motion technique for judging equiluminance. In: Mollon JD, Sharpe LT (eds) Colour Vision: Physiology and Psychophysics. Academic Press, London, pp 155-166

129. Robson AG, Harding G, van Kuijk F, Pauleikhoff D, Holder GE, Bird AC, Fitzke FW, Moreland JD (2005) Comparison of fundus autofluorescence and minimum-motion

measurements of macular pigment distribution profiles derived from identical retinal areas.

Perception 34: 1029-1034

130. West P, Mellerio J (2005) Measuring MPOD using a CRT display [online].

http://www.crsltd.com/research-topics/macular-pigment/index.html and

http://www.crsltd.com/catalog/metropsis/MP.html. Accessed 1 May 2009

131. Brindley GS, Willmer EN (1952) The reflexion of light from the macular and peripheral fundus oculi in man. J Physiol 116: 350-356

132. Berendschot T, DeLint PJ, van Norren D (2003) Fundus reflectance - historical and present ideas. Prog Retin Eye Res 22: 171-200

133. van Norren D, Tiemeijer LF (1986) Spectral reflectance of the human eye. Vision Res 26: 313-320

134. Delori FC, Pflibsen KP (1989) Spectral reflectance of the human ocular fundus. Appl Opt 28: 1061-1077

135. Elsner AE, Burns SA, Beausencourt E, Weiter JJ (1998) Foveal cone photopigment distribution: small alterations associated with macular pigment distribution. Invest Ophthalmol Vis Sci 39: 2394-2404

136. Berendschot T, Goldbohm RA, Klopping WAA, van de Kraats J, van Norel J, van Norren D (2000) Influence of lutein supplementation on macular pigment, assessed with two objective techniques. Invest Ophthalmol Vis Sci 41: 3322-3326

137. Bour LJ, Koo L, Delori FC, Apkarian P, Fulton AB (2002) Fundus photography for measurement of macular pigment density distribution in children. Invest Ophthalmol Vis Sci 43: $1450-1455$

138. Cardinault N, Gorrand JM, Tyssandier V, Grolier P, Rock E, Borel P (2003) Short-term supplementation with lutein affects biomarkers of lutein status similarly in young and elderly subjects. Exp Gerontol 38: 573-582

139. van de Kraats J, van Norren D (2008) Directional and nondirectional spectral reflection from the human fovea. J Biomed Opt 13: 024010

140. van de Kraats J, Berendschot T, van Norren D (1996) The pathways of light measured in fundus reflectometry. Vision Res 36: 2229-2247

141. Berendschot T, van Norren D (2005) On the age dependency of the macular pigment optical density. Exp Eye Res 81: 602-609

142. Kanis MJ, Berendschot T, van Norren D (2007) Influence of macular pigment and melanin on incident early AMD in a white population. Graefes Arch Clin Exp Ophthalmol 245: 767-773

143. Kanis MJ, Berendschot T, van Norren D (2007) Interocular agreement in melanin and macular pigment optical density. Exp Eye Res 84: 934-938

144. Berendschot T, Willemse-Assink JJM, Bastiaanse M, de Jong P, van Norren D (2002) Macular pigment and melanin in age-related maculopathy in a general population. Invest Ophthalmol Vis Sci 43: 1928-1932

145. Chang Y, Lee FL, Chen SJ, Chen SF (2002) Optical measurement of human retinal macular pigment and its spatial distribution with age. Med Phys 29: 2621-2628

146. Zagers NPA, van de Kraats J, Berendschot T, van Norren D (2002) Simultaneous measurement of foveal spectral reflectance and cone-photoreceptor directionality. Appl Opt 41: 4686-4696 
147. Zagers NPA, van Norren D (2004) Absorption of the eye lens and macular pigment derived from the reflectance of cone photoreceptors. J Opt Soc Am A Opt Image Sci Vis 21: 2257-2268

148. Kanis MJ, Wisse RPL, Berendschot T, van de Kraats J, van Norren D (2008) Foveal cone-photoreceptor integrity in aging macula disorder. Invest Ophthalmol Vis Sci 49: 20772081

149. Elsner AE, Burns SA, Delori FC, Webb RH (1990) Quantitative reflectometry with the SLO. In: Naseman JE, Burk ROW (eds) Scanning Laser Ophthalmoscopy and Tomography. Quintessenz-Verlag, Munich, pp 109-121

150. Seth R, Gouras P (2004) Assessing macular pigment from SLO images. Doc Ophthalmol 108: 197-202

151. Helb HM, Issa PC, Van der Veen RLP, Berendschot T, Scholl HPN, Holz FG (2008)

Abnormal macular pigment distribution in type 2 idiopathic macular telangiectasia. Retina 28: 808-816

152. van de Kraats J, Kanis MJ, Genders SW, van Norren D (2008) Lutein and zeaxanthin measured separately in the living human retina with fundus reflectometry. Invest Ophthalmol Vis Sci 49: 5568-5573

153. Sharifzadeh M, Bernstein PS, Gellermann W (2006) Nonmydriatic fluorescence-based quantitative imaging of human macular pigment distributions. J Opt Soc Am A Opt Image Sci Vis 23: $2373-2387$

154. Wolf-Schnurrbusch UEK, Roosli N, Weyermann E, Heldner MR, Hohne K, Wolf S (2007) Ethnic differences in macular pigment density and distribution. Invest Ophthalmol Vis Sci 48: 3783-3787

155. Bernstein PS, Delori FC, Richer S, van Kuijk FJM, Wenzel AJ (2010) The value of measurement of macular carotenoid pigment optical densities and distributions in agerelated macular degeneration and other retinal disorders. Vision Res 50: 716-728

156. Delori FC (1994) Spectrophotometer for noninvasive measurement of intrinsic fluorescence and reflectance of the ocular fundus. Appl Opt 33: 7439-7452

157. Delori FC, Dorey CK, Staurenghi G, Arend O, Goger DG, Weiter JJ (1995) In vivo fluorescence of the ocular fundus exhibits retinal pigment epithelium lipofuscin characteristics. Invest Ophthalmol Vis Sci 36: 718-729

158. von Ruckmann A, Fitzke FW, Bird AC (1995) Distribution of fundus autofluorescence with a scanning laser ophthalmoscope. Br J Ophthalmol 79: 407-412 159. Delori FC (2004) Autofluorescence method to measure macular pigment optical densities fluorometry and autofluorescence imaging. Arch Biochem Biophys 430: 156-162 160. Schmitz-Valckenberg S, Holz FG, Bird AC, Spaide RF (2008) Fundus autofluorescence imaging - review and perspectives. Retina 28: 385-409

161. Liew SHM, Gilbert CE, Spector TD, Mellerio J, Van Kuijk FJ, Beatty S, Fitzke F, Marshall J, Hammond CJ (2006) Central retinal thickness is positively correlated with macular pigment optical density. Exp Eye Res 82: 915-920

162. Trieschmann M, Heimes B, Hense HW, Pauleikhoff D (2006) Macular pigment optical density measurement in autofluorescence imaging: comparison of one- and two-wavelength methods. Graefes Arch Clin Exp Ophthalmol 244: 1565-1574

163. Jahn C, Wustemeyer H, Brinkmann C, Trautmann S, Mossner A, Wolf S (2005) Macular pigment density in age-related maculopathy. Graefes Arch Clin Exp Ophthalmol 243: 222-227

164. Liew SHM, Gilbert CE, Spector TD, Mellerio J, Marshall J, van Kuijk FJ, Beatty S, Fitzke F, Hammond CJ (2005) Heritability of macular pigment: a twin study. Invest Ophthalmol Vis Sci 46: 4430-4436

165. Delori FC, Goger DG, Dorey CK (2001) Age-related accumulation and spatial distribution of lipofuscin in RPE of normal subjects. Invest Ophthalmol Vis Sci 42: 1855-1866 166. Bernstein PS, Yoshida MD, Katz NB, McClane RW, Gellermann W (1998) Raman detection of macular carotenoid pigments in intact human retina. Invest Ophthalmol Vis Sci 39: 2003-2011 
167. Koyama Y, Takatsuka I, Nakata M, Tasumi M (1988) Raman and infrared spectra of the all-trans, 7-cis, 9-cis, 13-cis and 15-cis isomers of beta carotene - key bands distinguishing stretched or terminal-bent configurations from central-bent configurations. J Raman Spectrosc 19: 37-49

168. Ermakov IV, McClane RW, Gellermann W, Bernstein PS (2001) Resonant Raman detection of macular pigment levels in the living human retina. Opt Lett 26: 202-204 169. Bernstein PS (2002) New insights into the role of the macular carotenoids in agerelated macular degeneration. Resonance Raman studies. Pure Appl Chem 74: 1419-1425 170. Bernstein PS, Zhao DY, Wintch SW, Ermakov IV, McClane RW, Gellermann W (2002) Resonance Raman measurement of macular carotenoids in normal subjects and in agerelated macular degeneration patients. Ophthalmology 109: 1780-1787

171. Gellermann W, Ermakov IV, Ermakova MR, McClane RW, Zhao DY, Bernstein PS (2002) In vivo resonant Raman measurement of macular carotenoid pigments in the young and the aging human retina. J Opt Soc Am A Opt Image Sci Vis 19: 1172-1186

172. Gellermann W, Ermakov IV, McClane RW, Bernstein PS (2002) Raman imaging of human macular pigments. Opt Lett 27: 833-835

173. Zhao DY, Wintch SW, Ermakov IV, Gellermann W, Bernstein PS (2003) Resonance Raman measurement of macular carotenoids in retinal, choroidal, and macular dystrophies. Arch Ophthalmol 121: 967-972

174. Ermakov I, Ermakova M, Gellermann W, Bernstein PS (2004) Macular pigment Raman detector for clinical applications. J Biomed Opt 9: 139-148

175. Ermakov IV, Sharifzadeh M, Ermakova M, Gellermann W (2005) Resonance Raman detection of carotenoid antioxidants in living human tissue. J Biomed Opt 10: 064028 176. Hogg RE, Anderson RS, Stevenson MR, Zlatkova MB, Chakravarthy U (2007) In vivo macular pigment measurements: a comparison of resonance Raman spectroscopy and heterochromatic flicker photometry. Br J Ophthalmol 91: 485-490

177. Obana A, Hiramitsu T, Gohto Y, Ohira A, Mizuno S, Hirano T, Bernstein PS, Fuji H, Iseki K, Tanito M, Hotta Y (2008) Macular carotenoid levels of normal subjects and agerelated maculopathy patients in a Japanese population. Ophthalmology 115: 147-157 178. Sharifzadeh M, Zhao DY, Bernstein PS, Gellermann W (2008) Resonance Raman imaging of macular pigment distributions in the human retina. J Opt Soc Am A Opt Image Sci Vis 25: 947-957

179. Hogg RE, Zlatkova MB, Chakravarthy U, Anderson RS (2007) Investigation of the effect of simulated lens yellowing, transparency loss and refractive error on in vivo resonance Raman spectroscopy. Ophthalmic Physiol Opt 27: 225-231

180. Bernstein PS, Gellermann W (2003) Author response: assessment of the Raman method of measuring human macular pigment [Letter] [online]. Investigative Ophthalmology \& Visual Science. Available at: http://www.iovs.org/cgi/eletters?lookup=by date\&days=9999\#74. Accessed 15 September 2009

181. Bernstein PS, Gellermann W (2003) Author response: assessment of the Raman method of measuring human macular pigment (II) [Letter] [online]. Investigative

Ophthalmology \& Visual Science. Available at: http://www.iovs.org/cgi/eletters?lookup=by date\&days=9999\#74. Accessed 15 September 2009

182. Wooten BR, Hammond BR (2003) Assessment of the Raman method of measuring human macular pigment [Letter] [online]. Investigative Ophthalmology \& Visual Science. Available at: http://www.iovs.org/cgi/eletters?lookup=by date\&days=9999\#73. Accessed 15 September 2009

183. Wooten BR, Hammond BR (2003) Assessment of the Raman method of measuring human macular pigment (II) [Letter] [online]. Investigative Ophthalmology \& Visual Science. Available at: http://www.iovs.org/cgi/eletters?lookup=by date\&days=9999\#73. Accessed 15 September 2009

184. Hammond BR, Wooten BR (2005) Resonance Raman spectroscopic measurement of carotenoids in the skin and retina. J Biomed Opt 10: 054002 
185. Gellermann W, Bernstein PS (2006) Assessment of the validity of in vivo methods of measuring human macular pigment optical density [Letter]. Optom Vis Sci 83: 254-255

186. Hammond BR, Wooten BR, Smollon B (2006) Assessment of the validity of in vivo methods of measuring human macular pigment optical density - Authors' response [Letter]. Optom Vis Sci 83: 256-259

187. Beatty S, van Kuijk F, Chakravarthy U (2008) Macular pigment and age-related macular degeneration: longitudinal data and better techniques of measurement are needed. Invest Ophthalmol Vis Sci 49: 843-845

188. Bird AEC, Bressler NM, Bressler SB, Chisholm IH, Coscas G, Davis MD, Dejong P, Klaver CCW, Klein BEK, Klein R, Mitchell P, Sarks JP, Sarks SH, Sourbane G, Taylor HR, Vingerling JR (1995) An international classification and grading system for age-related maculopathy and age-related macular degeneration. Surv Ophthalmol 39: 367-374 


\title{
Measuring Macular Pigment Optical Density In Vivo: A Review Of Techniques
}

Olivia Howells, Frank Eperjesi, Hannah Bartlett

Ophthalmic Research Group, School of Life and Health Sciences, Aston University, Birmingham, B4 7ET, UK

Corresponding author: Olivia Howells; o.howells@aston.ac.uk; Tel. 0121204 4135;

Fax. 01212044048

\begin{abstract}
Background: Macular pigment has been the focus of much attention in recent years, as a potential modifiable risk factor for age-related macular degeneration. This interest has been heightened by the ability to measure macular pigment optical density (MPOD) in vivo. Method: A systematic literature search was undertaken to identify all available papers that have used in vivo MPOD techniques. The papers were reviewed and all relevant information was incorporated into this article. Results: Measurement of MPOD is achievable with a wide range of techniques, which are typically categorized into one of two groups: psychophysical (requiring a response from the subject) or objective (requiring minimal input from the subject). The psychophysical methods include heterochromatic flicker photometry and minimum motion photometry. The objective methods include fundus reflectometry, fundus autofluorescence, resonance Raman spectroscopy and visual evoked potentials. Even within the individual techniques, there is often much variation in how data is obtained and processed. Conclusion: This review comprehensively details the procedure, instrumentation, assumptions, validity and reliability of each MPOD measurement technique currently available, along with their respective advantages and disadvantages. This leads us to conclude that development of a commercial instrument, based on fundus reflectometry or fundus autofluorescence, would be beneficial to macular pigment research and would support MPOD screening in a clinical setting.
\end{abstract}

Keywords: fundus autofluorescence; fundus reflectometry; heterochromatic flicker photometry; macular pigment; macular pigment optical density; motion photometry; Raman spectroscopy. 


\section{INTRODUCTION}

Macular pigment is the collective name for three carotenoids, lutein, zeaxanthin and mesozeaxanthin, which are found at higher concentrations in the retina than anywhere else in the body, and to the exclusion of all other carotenoids [1]. They are only accessible to the body by dietary intake of foodstuffs or supplements containing them [2, 3], with high levels being found in certain fruits and vegetables, such as kiwi fruit, corn and spinach, as well as egg yolks [4].

Analysis of donor maculae is possibly the most unequivocal approach for assessing the distribution of macular pigment in the retina, and pioneering work by Snodderly and colleagues in the 1980 s achieved this $[5,6]$. Using primate monkeys and the technique of microdensitometry, it was confirmed, as expected, that macular pigment reaches its peak in the centre of the retina. There was then a sharp decline to negligible levels at approximately $1 \mathrm{~mm}\left(4^{\circ}\right)$ from the central fovea. In 1988, Bone et al., using high-performance liquid chromatography (HPLC), investigated the spatial distribution of macular pigment in human donors; in this case, it was found to reach negligible levels at $7^{\circ}$ eccentricity [7]. Within the retinal layers, macular pigment is primarily located in the photoreceptor axons and to a lesser extent in the inner plexiform layer [6, 8].

The macular carotenoids have an absorption spectrum of $400-540 \mathrm{~nm}$, peaking at approximately $460 \mathrm{~nm}$ [9]. This spectral peak, along with the spatial distribution and retinal layer localization of macular pigment contribute to its proposed function as a blue light filter. Short-wavelength (blue) light is more damaging to the retina than longer-wavelength light [10] so by attenuating the amount of blue light reaching the photoreceptors, macular pigment may protect the macula from this photo-damage; the higher the density of macular pigment (macular pigment optical density, or MPOD), the greater the amount of blue light filtering that will occur $[11,12]$. A second proposed function of macular pigment is that it protects the macula against oxidative stress by acting as an antioxidant [e.g. 13]. These blue light filter and antioxidant functions have led to the school of thought that having a high MPOD could help to protect against the eye disease age-related macular degeneration (AMD), the most prevalent cause of severe visual impairment in Western society [14-16]. As a result, there have been a multitude of studies investigating possible links between MPOD and AMD, using a variety of measurement techniques. Some of these studies have supported an MPOD-AMD association [e.g. 17, 18, 19] and some have not [e.g. 20, 21]. This inconsistent evidence is not too surprising, given the apparent multifactorial nature of AMD. What's more, 
it is highly likely that an individual's MPOD is equally multifactorial, but as one of the few potentially modifiable risk factors for AMD, its continued investigation is extremely important.

Macular pigment optical density may be measured in vitro or in vivo. In vitro measurement involves the techniques of HPLC [e.g. 22, 23] or microdensitometry [e.g. 24]. However, they can only be performed on excised retinas and so are clearly not suitable for widespread use. This review therefore details the most common techniques currently used to measure MPOD in vivo. These in vivo techniques are noninvasive and are normally categorized under one of two headings: psychophysical (requiring a response from the subject) or objective (requiring minimal input from the subject). Together they have established that MPOD varies widely between individuals, from virtually no macular pigment to greater than 1 log unit optical density, with average levels ranging from 0.16 [25] to 0.50 [26], depending on the method and/or the study population.

\section{METHOD}

A systematic literature search was conducted using ISI Web of Knowledge and PubMed. Key words and their combinations used for the search included 'macular pigment', 'macular pigment optical density', 'lutein', 'zeaxanthin', 'heterochromatic flicker photometry', 'motion photometry', reflectometry', 'autofluorescence', 'Raman', 'electrophysiology', and 'macular degeneration'. Further searches were undertaken for key researchers in the field such as Beatty, Berendschot, Bernstein, Bone, Delori, Gellermann, Hammond, Landrum, Moreland, Nolan, Robson, Snodderly, Stringham, Trieschmann, van de Kraats, and Wenzel. Further papers were obtained from the references of the retrieved articles. All the articles were reviewed and relevant information was incorporated into the manuscript.

\section{RESULTS OF REVIEW}

\section{Psychophysical techniques}

Psychophysical techniques of measuring macular pigment optical density (MPOD) include the following:

Threshold spectral sensitivity [e.g. 27, 28-31].

Colour matching [e.g. 32, 33-35].

Dichroism-based measurements [e.g. 9, 36].

Minimum motion photometry and apparent motion photometry [e.g. 37, 38-41]. 
Heterochromatic flicker photometry [e.g. 42, 43-53].

The first three of the psychophysical methods have now been largely superseded by heterochromatic flicker photometry (HFP) and, to some extent, minimum motion photometry. This is in part due to their increased level of difficulty and/or the longer time needed to perform them [54]. This review will therefore focus on the latter two methods. For information regarding the threshold sensitivity, colour matching and dichroism techniques, the reader is directed to the referenced studies, along with a validity review by Hammond et al. [54].

\section{Heterochromatic flicker photometry}

Developed by Ives in the early 1900s [55], HFP has so far been the most commonly used of all the techniques for measuring MPOD. As such, it is often used as a standard against which other techniques are validated [e.g. 56, 57-60], although at present there is no true 'gold-standard' in vivo measure of MPOD.

The use of HFP to measure macular pigment levels was first described over 30 years ago by Werner and Wooten [61] but the technique wasn't elaborated on until 1987, in a key paper by Werner, Donnelly and Kliegl [42]. Since then, HFP has been developed and used by numerous research groups investigating macular pigment. Key papers incorporating detailed descriptions and variations of the technique include Hammond and Fuld [43], Hammond et al. [44], Landrum et al. [45], Wooten et al. [46], Beatty et al. [47], Mellerio et al. [48], Bone and Landrum [49], Snodderly et al. [50], Tang et al. [62], lannaccone et al. [51], Stringham et al. [52], and van der Veen et al. [53]. All other studies using HFP to measure MPOD tend to use the instruments originally designed or developed by these investigators.

\section{Procedure}

In conjunction with many of the MPOD techniques, HFP exploits the spectral absorption properties and retinal location of macular pigment. Essentially, HFP determines MPOD by presenting a light stimulus of two alternating wavelengths at the fovea and at a parafoveal area. The wavelengths are chosen such that one is a short wavelength blue light that is maximally absorbed by macular pigment and the other is a longer wavelength green to yellow light that is not absorbed by macular pigment [6]. If the colours are alternated at an appropriate frequency and the luminance of the two colours is not perceived to be equal by the subject, the stimulus will appear as a flickering light; the perceived colour of this light will be an amalgamation of the two source colours [47, 49, 50,63]. Typically, the radiance (often 
also termed intensity) of the blue light is adjusted by the subject until the observed flicker is minimized [e.g. 44, 45, 46, 48, 50, 52, 62]. This occurs when there is an equiluminance match between the blue and green lights $[63,64]$. The procedure is then repeated at a parafoveal locus where macular pigment is negligible [65]. Since more blue light will be absorbed by macular pigment at the fovea than the parafovea, a greater radiance of blue light will be required at the fovea to appreciate minimal flicker. The log ratio of the radiance of blue light needed at the fovea compared with that needed at the parafovea gives a measure of peak MPOD (Formula 1), although whether this is truly the peak value is subject to discussion (see 'the edge hypothesis of heterochromatic flicker photometry').

MPOD $=\log \left(R_{\lambda_{s}}^{f} / R_{\lambda_{s}}^{p}\right)-\log \left(R_{\lambda_{\gamma}}^{f} / R_{\lambda_{\gamma}}^{p}\right)$

Formula 1. Example calculation for macular pigment optical density, from Stringham et al. [52]. $R_{\lambda_{s}}^{f}=$ radiance of a peak macular pigment absorption wavelength, e.g. $460 \mathrm{~nm}$, measured at a foveal location. $\mathrm{R}_{\lambda_{\mathrm{S}}}^{\mathrm{p}}=$ radiance of a peak macular pigment absorption wavelength, measured at a parafoveal location, e.g. $7^{\circ} . \mathbf{R}_{\lambda_{\gamma}}^{\dagger}=$ radiance of a negligible macular pigment absorption wavelength, e.g. $570 \mathrm{~nm}$, measured at a foveal location. $\mathbf{R}_{\lambda_{\gamma}}^{\mathrm{p}}=$ radiance of a negligible macular pigment absorption wavelength, measured at a parafoveal location.

\section{$\underline{\text { Instrumentation }}$}

Several variations on MPOD measurement by HFP have been developed since its first use in the 1970s. Traditionally, Maxwellian view devices have been used [e.g. 42, 43, 45]. These are complex optical systems that are not easily portable and which require the use of a dental bite bar. The bite bar keeps the subject's head stable so that their eye is correctly aligned with the incoming light beam $[46,65]$. Given the complexity of these devices, operators need a significant amount of training [46]. As a result, several research groups have simplified the optics and allowed the use of a free view, or Newtonian view, setup [e.g. $46,47,48,62]$. This negates the need for a bite bar, making the procedure more comfortable for the subject. Free view optical systems are also cheaper, easier to operate, and more portable (if not completely portable) than their Maxwellian counterparts [46, 47, 62]. Wooten et al. [46] demonstrated a strong correlation for mean and individual MPOD calculated between their free view system and an established Maxwellian view system $(r=$ 0.95 , no $p$-value provided). This showed that using the free viewing technique does not affect the accuracy of the derived result. Figures 1 and 2 depict a typical Maxwellian view 
optical system and free view optical system, respectively, whilst Table 1 summarizes their differences. It should be noted that slight variations in these differences do occur. For instance, the instrument developed by Beatty and co-workers [47] uses both a quartz halogen and light-emitting diode (LED) light source.

INSERT FIGURES 1 AND 2 ABOUT HERE

INSERT TABLE 1 ABOUT HERE (AFTER FIGURES 1 AND 2)

\section{TEST FIELDS}

The test fields are viewed at a near working distance, e.g. $33 \mathrm{~cm}$ [48]. Most devices use a central stimulus that corresponds to a visual angle of $1^{\circ}$ as standard, although there are exceptions; Landrum's $1.5^{\circ}$ [45] and Werner's $0.70^{\circ}$ [66], for instance. Moreover, many studies have used smaller test stimuli such as $12^{\prime}$ or $30^{\prime}$ when mapping the spatial profile of macular pigment $[42,44,49,50,52,60,64,67-79]$.

The wavelength chosen for the blue light has varied between researchers from $458 \mathrm{~nm}$ [e.g. $11,46,52,75,80,81]$ to $476 \mathrm{~nm}$ [17], and from $530 \mathrm{~nm}$ [e.g. 43, 82-85] to $575 \mathrm{~nm}$ [62] for the green light. Where the blue wavelength does not coincide exactly with the peak of macular pigment absorption, this should be accounted for in the final calculation of MPOD. This is of greater importance in the objective techniques where wavelengths are often further from the peak than those used in HFP. MPOD should also be adjusted according to the bandwidth of the light source; the narrower the bandwidth, the more accurately the measurement reflects MPOD at the particular wavelength [54]. For the HFP device first described by Wooten et al. [46], the LED with peak energy at $458 \mathrm{~nm}$ has a half-bandwidth of $20 \mathrm{~nm}$. As a result, MPOD must be increased by a $15 \%$ constant to correct for this [20, $50,54,77]$.

The peripheral reference measurement is usually made using the same test stimulus as used for the central measure, but the subject's gaze is directed to an eccentric fixation point. An exception to this is the Maculometer (developed by Mellerio et al. [48]) which instead turns the central $1^{\circ}$ field into a fixation point and presents an annular test field at $5.5^{\circ}$ from fixation. The subject therefore fixates centrally throughout the procedure. The authors reported that many subjects found this easier than maintaining an eccentric fixation. The parafoveal location used in different HFP apparatus varies from $4^{\circ}$ from the central fovea [e.g. $11,43,46,62,71,80,81,86]$ to $10^{\circ}[72,76]$ or $12^{\circ}[66]$. Similarly, the location of the peripheral point on the retina varies from temporal, nasal or superior retina, depending on instrument type. 


\section{FLICKER RATES}

The rate at which the blue and green lights are alternated is a difficult decision for researchers to make, since flicker sensitivity can vary between observers [65]. Ideally the flicker rate should allow for a suitable amount of null or minimal flicker to be achieved when adjusting the radiance of the test stimulus. If the flicker frequency is too low for an individual, they will have difficulty obtaining a point of null flicker. Conversely, if the flicker frequency is too high for an individual, they will have a wide range of null flicker, leading to variation in measurements $[50,52,63,75]$. There is also the need for rod and short-wavelength cone suppression to consider (see 'assumptions'). Until recently, most investigators have used set flicker frequencies that have varied from $11 \mathrm{~Hz}$ [e.g. 11, 57, 80, 81, 87, 88] to $30 \mathrm{~Hz}$ [e.g. 49, $68,89,90]$ in the fovea and from $6 \mathrm{~Hz}$ [e.g. $11,46,57,80,81,87,88$ ] to $25 \mathrm{~Hz}$ [e.g. 17, 47, $91]$ in the parafovea.

\section{BACKGROUND FIELDS}

Like test fields, the backgrounds upon which they are presented can also vary in size and wavelength between equipment. Sizes have ranged from $4^{\circ}$ in diameter [e.g. 43,82 ] to $30^{\circ}$ [53]. The colour of the background is invariably a blue wavelength or white. The purpose of these colours is discussed in the 'assumptions' section.

\section{RECENT DEVELOPMENTS}

In 2001, Moreland et al. described a novel method of measuring MPOD by HFP. They used the blue and green phosphor emissions of a colour computer monitor as stimuli for flicker minimization. Although perceptually distinct as blue and green, the broadband emission spectra of the phosphors resulted in a 50\% underestimation of MPOD. This was correctable with a model that incorporated these emission spectra and therefore allowed a way of calibrating the monitor. Bone and Landrum [49] questioned whether the retinal illuminance provided by the instrument was high enough to avoid rod intrusion. Apart from further use by Robson et al. [92] and Robson and Parry [41] this method of MPOD determination does not appear to have been widely used.

Snodderly et al. [50] paved the way for a new customized approach to HFP when they established a standardized protocol for measuring MPOD. The device used was a modified version of the one described by Wooten and colleagues in 1999, and included the addition of optimizing the flicker frequency for each individual. This was achieved by working out each subject's critical flicker frequency at the fovea and parafovea, then using an algorithm to 
determine the appropriate flicker rate to use when measuring MPOD. This procedure has since been adopted in other MPOD research [e.g. 51, 52, 64, 71, 72, 75, 76, 78, 79, 86, 93].

A new HFP technique called the macular assessment profile (MAP) test has been described by Rodriguez-Carmona et al. [94] and Kvansakul et al. [95]. The principle appears similar to the method detailed by Moreland et al. [38] and described above, in so far as the broadband phosphors of a visual display are employed and again require a correction model for this. The authors state that the test is a 'rapid and convenient' way of measuring a subject's macular pigment profile up to $8^{\circ}$ from the fovea, taking advantage of the ability of visual displays to produce stimuli of different sizes at randomized locations [94]. Although the test is said to have been validated, there does not appear to be any formally published data on this.

A further development for customized HFP was briefly described by Engles et al. [93] and elaborated upon by Nolan et al. [75] and Stringham et al. [52]. This involves the inverseyoking of the radiances of the blue and green stimuli so that the overall luminance of the test field remains constant, i.e. when the radiance of the blue stimulus is increased, the radiance of the green stimulus is proportionately decreased. As a result, potential distractions by changes in perceived brightness for the subject are avoided.

An entirely different approach to measurement of MPOD by HFP has been adopted in a new commercially available device which is described in detail by van der Veen et al. [53]. Instead of the subject responding to minimal or no flicker, they respond to the appearance of flicker as the alternation rate is decreased at $6 \mathrm{~Hz}$ per sec from a starting level of $60 \mathrm{~Hz}$. This is above the critical flicker fusion frequency for the test conditions and therefore subjects do not perceive any flicker initially. Rather than the radiance of one wavelength being adjusted by the observer, a sequence of blue-green ratios is used. These are inverse-yoked to ensure that overall luminance stays the same. With similarities to Snodderly et al. [50], the instrument determines each observer's sensitivity to flicker prior to the main part of the test. The technique also offers the possibility of estimating MPOD from a central measure alone, the peripheral measure being estimated from the age of the subject and their expected level of lens yellowing. A comparison between central and peripheral derived MPOD and estimated (central only) MPOD in 5616 eyes revealed a very close correlation $(r=0.92$, no p-value provided) [96].

Most recently, a further development on customized HFP and inverse yoking has been described by Connolly et al. [78] and Nolan et al. [97]. Whilst sticking with the traditional 
method of HFP MPOD determination, i.e. the radiance of blue light being increased/decreased until the point of minimal flicker, it takes on a more automated approach, as per the device of van der Veen et al. [53]. The instrument's electronics increase/decrease the amount of blue in the stimulus at a set rate. This removes any interindividual variability in the speed at which the blue-green ratio is adjusted, therefore improving the accuracy of the MPOD value.

\section{Assumptions}

The HFP measurement of MPOD relies on several assumptions. Many of these assumptions are largely accepted because of the close relationship between HFP-derived macular pigment spectral absorption curves and spectral curves derived in vitro. Nevertheless, some of the main assumptions are described:

1. Absorption or scattering properties of the ocular media being accounted for through use of a parafoveal locus $[47,49,56,98]$. Essentially, this means that the amount of yellowing in the media (e.g. the crystalline lens) would influence the measured MPOD value but the reference measure outside of the fovea cancels this effect [96]. This is demonstrated by Formula 2, below. Evidence that this assumption is correct comes from real and simulated data. For instance, Ciulla et al. [81] measured MPOD in 24 patients before and after cataract surgery. No significant difference in MPOD pre or post surgery was found, indicating that varying degrees of crystalline lens absorption does not affect macular pigment measurement when the HFP method is used. Wooten et al. [46] simulated clear and dense lenses by incrementally altering the background field radiance of their free view device. No significant differences in MPOD were found. Most recently, Makridaki et al. [96] demonstrated on a new HFP instrument [53] that lens yellowing, whether simulated or real, had no effect on the measured MPOD.

$B$ fov $x T_{\text {lens }} \times T_{M P}=B$ ref $x T_{\text {lens }}$

$\mathrm{T}_{\mathrm{MP}}=\mathrm{B}$ ref $/ \mathrm{B}$ fov

$M P O D=\log 1 / T_{M P}=\log (B$ fov $/ B$ ref $)$

Formula 2: Macular pigment optical density derivation, from Snodderly and Hammond [65]. In addition to the transmission of blue light through the macular pigment $\left(T_{M P}\right)$, the transmission through the lens ( $T_{\text {lens }}$ ) is taken into account. $B$ fov and $B$ ref are the radiances 
of blue light needed to minimize flicker at the fovea and reference point, respectively. Since $T_{\text {lens }}$ is assumed to be the same at both the fovea and reference, it is removed from equation (i). The final equation (iii) is a simplified version of Formula 1.

2. Accurate subject fixation and response. This is partly checked through assessment of instrument reliability. Werner et al. [42] also checked fixation accuracy on four of their subjects (age range 15 to 71 ) with an additional test; all subjects were able to accurately fixate to within $\pm 1.00^{\circ}$ of the foveal and parafoveal stimuli, or better, providing further evidence for this particular assumption.

3. Equal spectral sensitivity and distribution of photoreceptors across the retina, such that the difference ratio between the foveal and parafoveal locations is dependent solely on the macular pigment $[12,42,65]$. This assumption is not correct but is accounted for with the design of HFP instrumentation. Rods and short-wavelength sensitive cones (S-cones) are absent at the fovea, whilst being abundant in the peripheral retina and parafovea, respectively [99, 100]. Conversely, medium-wavelength cones (M-cones) and longwavelength cones (L-cones) are present in much higher concentration in the fovea than elsewhere [101]. However, unlike rods and S-cones, the ratio of $M$ to $L$ cones has been shown to remain fairly constant, at least in the central retina [102-104] and, as a result, should not affect the measured MPOD. It is therefore generally accepted that removing the rod and S-cone contributions is of greater importance. To do this, investigators have designed their HFP apparatus accordingly. The background field is often blue to suppress the S-cone population [e.g. 42, 43, 44, 46, 48, 50-52, 62, 105] or bright white to provide photopic conditions and hence suppress the rod population [e.g. 45, 49, 53]. The flicker frequency is chosen so that it is high enough to further exclude rods and S-cones. This is achieved because the flicker rate is above the critical flicker fusion frequency (the alternation rate at which a flickering light is no longer resolvable by the visual system and thus appears steady to an observer) of rods and S-cones, but is still lower than the critical flicker fusion frequency of $\mathrm{M}$ - and L-cones $[65,106,107]$.

4. The peripheral reference locus having a negligible level of macular pigment. Some studies have questioned this assumption [39, 94, 108-112], particularly when eccentricities as little as $4^{\circ}$ from the fovea have been used $[11,20,43,46,62,71,80,81,86-88,113]$. HFP spatial distribution plots of macular pigment (see 'validity and reliability') have gone some way to disproving any concerns, such that for most individuals, the assumption holds.

\section{Validity and reliability}


The validity and reliability of a technique are two important but quite different issues. Reliability, as described by Gallaher et al. [114], refers to the 'ascertainment of the reproducibility of a given measurement on the same subject at two distinct points in time'. Although this is a very important aspect of any instrument, it does not automatically imply that the instrument is valid. This was well illustrated by Snodderly et al. [50], where it was pointed out that one observer had a repeatable negative value of MPOD. The measure was therefore reliable but was nevertheless of questionable validity. For in vivo measurement of MPOD, validity is demonstrated by showing a matching comparison with the known spectral and spatial properties of macular pigment in vitro. Some studies have done this indirectly by comparing the measurements from a new device with those of an older, more established device $[46,56-59]$.

\section{VALIDITY}

As mentioned above, the validity of MPOD measurement by HFP can be assessed in two main ways $[52,71]$. Either by deriving the spatial profile of macular pigment across the fovea [e.g. $42,44,50,52,53,67,70,71,75]$ and comparing that to in vitro knowledge of macular pigment distribution [e.g. 5, 7, 24, 115], or by deriving the spectral absorption profile of macular pigment $[17,42,43,47,49,52,71]$ and comparing that to the shape of a known in vitro absorption curve of macular pigment. The latter method is considered to be more robust than the former, since spatial profile can vary between individuals $[44,50,59,64,71$, $75,78,116,117]$.

Spatial profiles of MPOD are achieved by altering the size and/or the eccentric position of the test stimulus, thus producing a curve that can be used to describe the change in MPOD with increasing eccentricity from the central fovea. An example is shown in Figure 3.

\section{INSERT FIGURE 3 ABOUT HERE}

Spectral absorption profiles of MPOD are obtained by systematically altering the wavelength of the test stimulus. The procedure is the same as that used to determine peak MPOD, except that a number of light wavelengths must be alternated with the reference stimulus until minimum flicker is accomplished, instead of the blue light alone. The extra measurements increase the duration of the test but permit a curve of spectral absorption to be plotted, see Figure 4. The in vivo HFP-generated curve is simultaneously compared with the shape of an in vitro spectral curve of lutein and zeaxanthin. The choice of which in vitro curve to use is not an easy one, since the ideal comparison of in situ spectral data from 
human retinas does not currently exist [54]. Consequently, different researchers have used different data. These include: Wyszecki and Stiles' composite data curve ${ }^{1}$ [118], used by Werner et al. [42], Hammond and Fuld [43], Beatty et al. [47], and Beatty et al. [17]; Snodderly and colleagues' microspectrophotometry-derived data from primate monkeys [6], used by Hammond and Fuld [43]; and spectral measures of lutein and zeaxanthin dissolved in olive oil [24] or incorporated in liposomes [9]. These last two in vitro spectrums have more recently been used in combination when assessing validity $[52,54,71]$.

The outcomes of these validity tests have shown a good correlation with in vitro MPOD distribution and absorption [e.g. 71] and therefore attest to the validity of HFP as a measurement method for MPOD.

INSERT FIGURE 4 ABOUT HERE

${ }^{1}$ The data from Wyszecki and Stiles [118] is actually a weighted composite curve of six psychophysical methods that had been used up to that time, rather than in vitro data. 
RELIABILITY

Numerous researchers, through test-retest checks, have assessed the reliability of HFP measurement of macular pigment. The statistical descriptors used to evaluate reliability vary between studies. Table 2 is a summary of all available information on HFP test-retest reliability indicators. It shows that for most subjects, HFP provides repeatable measures of MPOD and is therefore a reliable technique. Interestingly, the two most recent evaluations of HFP appear to give the weakest indication of its reliability $[119,120]$. These studies were independent of each other but used the same HFP device, one of the first that has been designed for use in a clinical setting rather than a research setting. The results suggest that further developments may be required for this particular HFP instrument in order to verify its suitability to accurately assess MPOD.

\section{INSERT TABLE 2 ABOUT HERE}

\section{The edge hypothesis of heterochromatic flicker photometry}

As mentioned previously, most instruments use a stimulus size of $1^{\circ}$ to measure MPOD. One might reasonably assume that this measures the total amount of macular pigment across the whole $1^{\circ}$ area or the peak level of macular pigment. However, many investigators disagree with both these assumptions and instead believe that the level of calculated MPOD is mediated by the edge of the stimulus [e.g. $11,42,44,48,50,52,121$ ], so that for a $1^{\circ}$ stimulus, the recorded MPOD corresponds to the macular pigment level at $0.5^{\circ}$ from the centre of the fovea. Werner et al. [42] were the first to suggest this theory and it gained momentum when Hammond et al. [44] found a very high correlation $(r=0.91, p<0.00001)$ between MPOD measured with a $1^{\circ}$ test stimulus and a point test stimulus of $12^{\prime}$ placed at $0.5^{\circ}$ (see Figure 5). Their data also indicated that MPOD at $0.5^{\circ}$ (as measured with a $1^{\circ}$ stimulus) is an estimated $69 \%$ of the true peak MPOD. For example, an MPOD value of 0.4 measured with a $1^{\circ}$ test would indicate a peak macular pigment density of 0.58 . The 'edge hypothesis' has been questioned, however, most notably by Bone et al. [68]. In contrast to Hammond et al. [44], they found that the measured MPOD corresponds to the level of macular pigment at approximately $50 \%$ of the stimulus radius (Figure 6). Further evaluation indicated that this equated closely to the average amount of macular pigment over the whole stimulus area [68]. Nevertheless, in spite of this conflicting evidence, most researchers have continued to assume the edge hypothesis in their HFP work [e.g. 60, 63, 64, 73-75, 78, 122] and this would appear to be a reasonable decision, with further evidence for it coming most recently from van der Veen et al.[121]. 
INSERT FIGURES 5 AND 6 ABOUT HERE

\section{Advantages and disadvantages of HFP}

Advantages include: 1) no pupil dilation required 2) inexpensive equipment relative to objective techniques 3 ) independence from absorption and scattering properties of the ocular media 4) good test-retest reliability on many subject populations 5) proven validity. Disadvantages include: 1) some subjects finding HFP difficult to carry out, especially the peripheral task, which is subject to Troxler's effect - a perceptual fading of peripheral stimuli [123]; Troxler's effect also becomes more distracting the more eccentric the peripheral target is, making the use of eccentricities certain to have no macular pigment difficult to record accurately 2) a long testing time if complete spectral and/or spatial distribution is required 3) unsuitability for some individuals, such as young children, people with learning difficulties or people with insufficient visual acuity or visual fields.

\section{Motion photometry}

The minimum motion paradigm was initially described by Stumpf [124], although this went largely unnoticed until its translation into English by Todorović in 1996 [40, 125]. With parallels to HFP, it refers to the perceived reduction in motion of a moving square or sine wave grating as equiluminance of the colours involved is reached. The concept was taken up for use in photometry by both Moreland [126, 127] and Anstis and Cavanagh [128], but in subtly different ways. This then led to the use of minimum motion photometry for in vivo measurement of macular pigment [e.g. 33, 37].

\section{Procedure}

Many of the principles described for HFP also apply for motion photometry measurement of MPOD, i.e. a wavelength of light at the peak of macular pigment absorption is compared with a wavelength of light not absorbed by macular pigment, at central and parafoveal locations. Moving square wave gratings are used, with the bars being alternately illuminated by the two light wavelengths. The radiance of the longer wavelength stimulus is adjusted until the motion appears to slow down or change direction, depending on the method being employed [126-128]. The slowing down of the grating is minimum motion photometry, whereas the reversal of grating movement is known as apparent motion photometry [40]. As with HFP, different radiances of the test wavelength will be required for equiluminance at the 
foveal and parafoveal positions, on account of the higher levels of macular pigment at the fovea. A log ratio of these radiances provides a measure of MPOD [37-41, 92, 129].

\section{$\underline{\text { Instrumentation }}$}

The motion photometry technique for measurement of MPOD has not been as widely used as HFP. Apart from the fundamental differences between classic minimum motion photometry and apparent motion photometry (explained below), there is little variation in the instrumentation that has been adopted.

\section{MINIMUM MOTION PHOTOMETRY}

Minimum motion photometry for MPOD, as described and used by Moreland, Robson and colleagues [e.g. 37, 38-41, 92, 129], employs a Moreland anomaloscope (traditionally used for colour vision assessment) that is adapted to produce a moving square wave grating with a spatial frequency of 0.38 cycles per degree. A rotating spiral mirror generates the grating which, when viewed through a circular or annular stop, appears to move horizontally across the visual field. The bars of the grating are alternately illuminated with two narrow-band interference filters from a single tungsten-halogen lamp. The interference filters typically provide wavelengths of $460 \mathrm{~nm}$ (blue - maximal absorption by macular pigment) and 580 $\mathrm{nm}$ (yellow - negligible absorption by macular pigment). Luminance matched filters of 450 $\mathrm{nm}$ are added to the grating bars to create a background pedestal that saturates S-cones (see 'assumptions'). The grating moves at a constant velocity of $14 \mathrm{~Hz}$; this also rules out any rod or S-cone contribution.

Unlike HFP, it is the norm with motion photometry for the spatial profile of macular pigment to be plotted, rather than peak MPOD alone. Consequently, the test fields comprise up to two central, circular stops of $0.8-0.9^{\circ}$ (visual angle) and $2.2^{\circ}$, and 11 annular stops placed eccentrically from $0.8^{\circ}-7.5^{\circ}$ in the superior visual field. The setup is illustrated in Figure 7. The minimum motion technique described here uses a Maxwellian view system but does not require a bite bar; rather, an adjustable chin rest (vertically and horizontally) is used for pupil centration [40].

\section{INSERT FIGURE 7 ABOUT HERE}

\section{APPARENT MOTION PHOTOMETRY}


Apparent motion photometry, based on the phenomenon detailed by Anstis and Cavanagh [128], has been developed into a commercially available device for MPOD assessment by West and Mellerio [130]. However, there do not appear to be any peer-reviewed studies on the instrument. The instrumentation details given below are therefore based on information from the Cambridge Research Systems' (CRS) website (http://www.crsltd.com/catalog/metropsis/MP.html).

Instead of a Moreland anomaloscope, a cathode ray tube (CRT) monitor is used. Four square wave gratings on a blue background are presented sequentially, 90 degrees out of phase with each other. The first and third gratings are made up of blue and red bars, produced by the blue and red phosphors of the CRT; blue for maximal macular pigment and red for negligible macular pigment absorption. The second and fourth gratings are achromatic, being composed of light and dark grey bars, i.e. luminance gratings. The apparent motion paradigm dictates that when the luminance of the red bars is greater than the blue, the red bars appear to jump rightwards to the light grey bars in the luminance grating, and when the luminance of the blue bars is greater than the red, the blue bars appear to jump leftwards to the light grey bars in the luminance grating (Figure 8, left). The subject therefore sees movement in one of two directions. The setup of West and Mellerio's device results in these directions being up or down (Figure 8, right). The red luminance is adjustable, and at the point of red-blue equiluminance, the direction of motion becomes 'ambiguous'. A two-alternative forced choice procedure is used to determine equiluminance (and subsequent MPOD) at central and eccentric locations. The system crosses over the threshold several times and can provide the standard deviation of each MPOD measurement. The grating alternation frequency must be optimized, usually between 8 and $20 \mathrm{~Hz}$, for each subject for reliable results.

\section{INSERT FIGURE 8 ABOUT HERE}

Like minimum motion, the apparent motion photometer for MPOD routinely plots the spatial distribution of macular pigment. Two central vertical strips $\left(0.3^{\circ} \times 1.25^{\circ}\right)$ are located at $0^{\circ}$ and $1^{\circ}$, whilst six $45^{\circ}$ annular test fields are located from $2^{\circ}-7^{\circ}$ from fixation. A shorter threelocation test is also available.

With a CRT monitor comes the problem of spectral overlap of the red, green and blue phosphors, similar to the problems of the colour monitor for HFP discussed in HFP 'recent developments' $[38,41,92]$. The apparent motion photometer overcomes this with the use of 
an optical filter that blocks light between 460 and $640 \mathrm{~nm}$, thus avoiding a significant underestimate of MPOD.

\section{Assumptions}

The same assumptions apply to minimum motion photometry as for HFP, namely absorption or scattering properties of the ocular media being accounted for through use of a parafoveal locus; accurate subject fixation and response; equal spectral sensitivity and distribution of Land $\mathrm{M}$-cones across the retina; and the peripheral reference locus having a negligible level of macular pigment.

The apparent motion device offers an interesting solution to accurate fixation by using a video gaze tracking system that inhibits stimulus presentation whenever fixation is not maintained to a sufficient level (within $\pm 0.5^{\circ}$ of target). This offers an improvement over unmonitored free view techniques whilst avoiding the need for a Maxwellian view system. With respect to L- and M-cone distribution, Robson et al. [129] used a 460/550 nm combination as well as the customary $460 / 580 \mathrm{~nm}$ to see if there was any variation in MPOD measurements. The correlation was extremely strong (slope $=1.00, r=0.99$ ), indicating that even if the distribution does vary with eccentricity, the effect is likely to be very small [129]. Like HFP, motion photometry also uses a blue background and a suitable temporal frequency to ensure rod and S-cone suppression. The assumption of the peripheral reference eccentricity having negligible macular pigment should hold for most individuals because spatial distribution is always plotted out as far as 7 or 8 degrees with motion photometry.

\section{Validity}

We could find no evidence of motion photometry being used to derive spectral profiles of macular pigment. As a result, it is difficult to know the true validity of the technique, although average MPOD values and spatial profiles are in line with HFP, and consistent correlations with the autofluorescence method of macular pigment measurement [39, 129] and two HFP devices $[38,48]$ have been found. Nevertheless, questions regarding the validity of motion photometry for measurement of macular pigment have been raised [54].

$\underline{\text { Reliability }}$ 
The reliability of minimum and apparent motion photometry has not been as vigorously assessed as it has been with HFP. The average of five readings is taken at each location $[37,39,41,92,129]$ and repeated measurements have been incorporated in averaged results [39]. However, there does not appear to be any published statistical data on testretest reliability.

\section{The non-edge hypothesis of motion photometry}

It was explained earlier that most researchers using HFP assume an edge hypothesis with MPOD. In minimum motion photometry, data analysis has led to the support of a non-edge hypothesis $[34,39,40]$. To reiterate, this would mean that when a circular, foveal stimulus is used, the measured MPOD would not represent the amount of macular pigment at the stimulus radius. In the case of motion photometry, researchers believe that the measured MPOD actually represents the amount of macular pigment at approximately $70 \%$ of the stimulus radius $[34,40]$. Subsequently, the 'peak' MPOD value using the smaller 0.8-0.9 central stimulus has been plotted at $0.3^{\circ}$ eccentricity from the fovea [e.g. 39, 41, 129].

\section{Advantages and disadvantages of motion photometry}

As a psychophysical technique, motion photometry has much in common with HFP in terms of pros and cons. There is no need for pupil dilation, any interference from the ocular media is accounted for and it is a relatively straightforward test for subjects to partake in. On the down side, Troxler's effect remains a problem for some individuals, and good comprehension of the task is required, so it is not suitable for everyone. No information is provided in any motion photometry studies regarding the level of visual acuity of subjects; therefore it is unknown whether reliable MPOD measurements are achievable on subjects with lower than normal acuity.

\section{Objective techniques}

The objective techniques for measuring MPOD are:

Fundus reflectometry

Fundus autofluorescence

Resonance Raman spectroscopy

Electrophysiology using visual evoked potentials 


\section{Fundus reflectometry}

Quantitative measurement of light reflected from the fundus is known as fundus reflectometry (FR), and the researchers Brindley and Willmer [131] were the first to adopt this technique. Their aim was to estimate MPOD in vivo by comparing light reflected at the macula with light reflected from a peripheral area of retina. Since then, FR has gone on to become the most widely used of the objective methods for MPOD measurement, although many improvements and variations have been developed along the way.

\section{Procedure}

When light enters the eye it has many structures to pass through, including the cornea, the crystalline lens, the retina and the choroid. Some of these structures (and their components) will reflect a small part of the light, whilst others will absorb part of it. Through measurement of reflected light from the retina and choroid, FR is able to assess several ocular features, including macular pigment [109]. A thorough history of FR is provided by Berendschot, DeLint and van Norren [132]; this review will be limited to the use of FR in measuring MPOD.

Although there are several variations on the reflectometry procedure, there are two methods that predominate. The first is a comparison technique, similar to that used in HFP. Light reflected from the fovea is compared with light reflected from an eccentric retinal area, using two wavelengths (one absorbed by macular pigment and one not) or using a spectrum of wavelengths. Since macular pigment absorbs rather than reflects certain wavelengths there will be a difference in the observed reflectances at the fovea and periphery, owing to the assumed lack of macular pigment at the eccentric site. Researchers who have used this method include Brindley and Willmer [131], van Norren and Tiemeijer [133], Delori and Pflibsen [134], Elsner et al. [135], Berendschot et al. [136], Delori et al. [56], Bour et al. [137], Wüstemeyer et al. [98], and Cardinault et al. [138].

The second core technique is known as a spectral analysis [109]. As the name suggests, this involves the analysis of a spectrum of reflected light from a spot of light on the retina. To achieve this, a detailed optical model of the pathways of light in the eye is required. $A$ number of optical models of increasing complexity have been proposed over the years, from van Norren and Tiemeijer [133] through to van de Kraats and van Norren [139]. Probably the most familiar optical model is that derived by van de Kraats, Berendschot and van Norren [140], which has been used to work out MPOD in several studies [e.g. 58, 136, 141-144]. In 
essence, the density of macular pigment is determined using its known spectral characteristics and by taking into account the amount of light reflected at the internal limiting membrane, the photoreceptor discs and the sclera [58, 136, 140, 141, 143]. The densities of the lens, melanin and blood are likewise calculated.

Even with these two quite separate forms of FR, there is often an overlap between the two, as demonstrated in the following section.

\section{$\underline{\text { Instrumentation }}$}

Many instruments have been used for FR and it is beyond the scope of this review to explain them all. Consequently, only the more recent fundus reflectometers will be described in any detail. However, common to most methods of FR is the need for pupil dilation, a bleaching of the visual pigments prior to measurement, and some form of head stabilization, either with a bite bar or with a chin rest and temple pads.

Equipment for reflectometry can be broadly categorised into modified fundus cameras, purpose-built reflectometers and modified scanning laser ophthalmoscopes.

\section{FUNDUS CAMERAS}

Many investigators have used modified fundus cameras to measure MPOD, including van Norren and Tiemeijer [133], Delori and Pflibsen [134], Chen, Chang and Wu [108], Bour et al. [137], Chang et al. [145], Neuringer et al. [3], and Bone, Brener and Gibert [59]. Of these, some have used the comparison technique, some spectral analysis and others a mixture of the two. Chen et al. [108], for example, used an optical model as per the spectral analysis technique but only two wavelengths $(460 \mathrm{~nm}$ and $560 \mathrm{~nm}$ ) rather than a full spectrum of wavelengths. In brief, their setup, like several others, consisted of a fundus camera connected to a cooled CCD (charge-coupled device). The filter normally used to take redfree photographs was replaced with narrow band interference filters of 460 and $560 \mathrm{~nm}$, i.e. maximal and minimal macular pigment absorption. Following pupil dilation, the subjects were instructed to fixate a dim red dot with the eye not being tested whilst the measured eye was slowly light adapted in order to bleach nearly all the photoreceptor pigments [56, 108, 146]. Two fundus pictures, one taken at each of the two wavelengths, were manually aligned using retinal landmarks. Using a chosen optical model, MPOD at each pixel point in the retina was calculated. In this way, Chen et al. were also able to plot the spatial distribution of macular pigment across the central retina. 
Recently, Bone et al. [59] described a modified fundus camera that does not require pupil dilation or bleaching of the photoreceptor pigments.

\section{PURPOSE-BUILT REFLECTOMETERS}

The most recent 'purpose-built reflectometers' are the Foveal Reflection Analyzer (FRA), originally developed by Zagers et al. [146] and the Macular Pigment Reflectometer (MPR), originally developed by van de Kraats et al. [117].

The diagrammatic setup of the FRA is shown in Figure 9. After dilation, subjects fixate a central cross hair (Figure 9b). In the original instrument (FRA 1), light from a halogen lamp is directed into the eye as a Maxwellian view system with an entrance pupil of $0.8 \times 1.2 \mathrm{~mm}$. This illuminates a $2.8^{\circ}$ spot on the central fovea, of which the middle $1.9^{\circ}$ is used for analysis. A video observation channel of the pupil and retina helps alignment as well as allowing monitoring of subject fixation. An imaging spectrograph collects the reflected light from the $1.9^{\circ}$ area and focuses it onto a cooled CCD camera. The spectrograph has a slit that creates a $0.8 \times 12 \mathrm{~mm}$ exit pupil above the smaller entrance pupil, and its spectral range is 420 to $790 \mathrm{~nm}$, thus allowing a spectral analysis of the reflected light using one of the optical models referred to earlier. The FRA 1 has been used to investigate MPOD in studies by Zagers and van Norren [147] and Berendschot and van Norren [141]. Berendschot and van Norren [141] also used a newer version of the device, the FRA 2, which has a number of differences from the first version, including being smaller, which makes it desktop mountable. Kanis et al. [143, 148] and van de Kraats and van Norren [139] have also used the FRA 2 in their studies.

\section{INSERT FIGURE 9 ABOUT HERE}

The diagrammatic setup of the MPR is shown in Figure 10. Like the FRA, the MPR, as described by van de Kraats et al. [58], involves a 30W halogen lamp directed into the pupil, a separated exit pupil and a spectrometer, i.e. spectrograph. The spectrometer has a spectral range of 400 to $800 \mathrm{~nm}$. The subject is asked to fixate the centre of a $1^{\circ}$ incoming light beam. This incoming illumination forms a $1^{\circ}$ spot on the central fovea; the reflected light is also collected over the same $1^{\circ}$ area and analyzed using an optical model. The MPR is the first reflectometry device that has a proven ability to measure MPOD through an undilated pupil, provided the pupil is $3 \mathrm{~mm}$ or larger; van de Kraats et al. [58] found no significant difference between their MPOD measurements for dilated and undilated pupils in 20 subjects. A further development of the MPR, recently reported by van de Kraats et al. 
[139], is the means to measure the individual optical densities of lutein and zeaxanthin, the components of macular pigment.

\section{INSERT FIGURE 10 ABOUT HERE}

\section{SCANNING LASER OPHTHALMOSCOPES}

Elsner et al. [149] were the first to use a scanning laser ophthalmoscope (SLO) for the purpose of measuring MPOD. Since then it has become a popular FR method for measuring macular pigment [e.g. 25, 98, 116, 136, 141, 150]. Some SLOs have been custom-built for MPOD measurement and as such are not accessible to most clinicians [135, 136]. However, Wüstemeyer et al. [98] modified a commercially available SLO, allowing reflectance images to be recorded with an argon laser at wavelengths $488 \mathrm{~nm}$ and $514 \mathrm{~nm}$, with a fast switch between the two. They used the comparison technique, with an eccentric reference point of $14^{\circ}$ from the fovea. MPOD in a $2^{\circ}$ central fovea test field was calculated as follows (Formula 3):

MPOD $=\mathrm{C}_{\lambda}{ }^{*}\left[\log \left(\operatorname{Ref}_{514, \text { foveal }} / \operatorname{Ref}_{488, \text { foveal }}\right)-\log \left(\operatorname{Ref}_{514, \text { parafoveal }} / \operatorname{Ref}_{488, \text { parafoveal }}\right)\right]$

Formula 3. Calculation of macular pigment optical density, from Wüstemeyer et al. [98]. $C_{\lambda}=$ constant, dependent on the absorption coefficients of macular pigment. $\operatorname{Ref}_{514}$ and $\operatorname{Ref}_{488}=$ reflectances measured at 514 and $488 \mathrm{~nm}$.

Using a foveal and parafoveal comparison is not common to all SLOs when measuring MPOD. Berendschot and van Norren [116, 141], for instance, used the same two wavelengths as Wüstemeyer et al. [98] but did not use any specific eccentric reference point and therefore produced density maps of the sum of both the lens and macular pigment.

One of the main advantages of using a SLO over other FR techniques is its confocal optics, which help minimize stray light scatter, the biggest hindrance in FR. This will be elaborated on in the 'assumptions' section.

\section{TEST FIELD VARIATIONS}

The size of the detection field chosen to measure peak MPOD varies not only between the three categories of reflectometer but also within the categories, from $0.5^{\circ}[116,141]$ to $2^{\circ}$ $[25,56,98,138]$ and $2.5^{\circ}$ [133]. Likewise, when two wavelengths corresponding to high and low macular pigment absorption are used, as is often the case for modified fundus cameras, the chosen wavelengths differ slightly between equipment, as they do in HFP devices. In 
SLOs, the two wavelengths are always 488 and $514 \mathrm{~nm}$ because these are the two most appropriate argon laser lines. The deviation of these laser lines from the true maximum and minimum of macular pigment absorption ( 460 and $>530 \mathrm{~nm}$, respectively [54]) requires that a correction is made to account for this in the final MPOD estimation [116, 136, 141, 151], although it is not clear whether all research groups actually do this. For the comparison technique when a peripheral reference point is used, the chosen eccentricity has ranged from as little as $4^{\circ}$ from the central fovea [137] up to $14^{\circ}[98,136]$.

\section{Assumptions}

The assumptions for FR are not as openly explained in the literature as for HFP. Nonetheless, several of the more commonly noted assumptions are highlighted below:

1. Homogeneity of fundus tissues. The spectral characteristics, absorption, reflection and scattering properties of the various retinal tissues (e.g. melanin) are assumed to be homogenous across the areas being assessed. Gellermann and Bernstein [111], among others, point out that this is a simplification. However, most researchers [e.g. 58] do not consider this to be a problem, and with good reason it would seem. For instance, the effect of irregular RPE melanin distribution on measured MPOD was investigated by Delori et al. [56]. They concluded that it had no strong effect on MPOD as measured with their reflectometry technique.

2. Bleaching of photoreceptor pigments. It has been established that $93-99 \%$ of cone photopigment and $59-85 \%$ of rod photopigment is bleached as a result of the level of illumination used prior to measurement, depending on the particular reflectometry method used $[56,58,108,137]$. Bleaching is important to avoid light absorption by the pigments and their subsequent interference with MPOD. It is assumed that any remaining unbleached photopigment, particularly rhodopsin (the pigment in rods), has a minimal effect. This has been investigated by Chen et al. [108], Delori et al. [56] and Bour et al. [137] and proven to be the case.

3. Light scatter accounted for. If reflectance from pre-retinal and intra-retinal structures are not controlled for, the measured MPOD can be artificially low [56, 109, 141]. This is because the reflectance method works on the principle that all the incident light is reflected after passing through the macular pigment. If some light is reflected before it reaches the macular pigment, e.g. by the crystalline lens, then this will be collected as reflected light but it won't actually have been affected by macular pigment absorption, thus leading to an erroneously 
low MPOD [25]. Most reflectometry devices do aim to eliminate this problem, although it would appear that some are more successful than others, judging by the lower than 'average' MPOD estimates found in some studies [e.g. 25, 56, 98, 137]. Methods used to allow this assumption to hold as far as possible include separating the entrance and exit pupils, using confocal optics as found in SLOs, and the incorporation of stray light into optical models. In addition, for the comparison technique of $\mathrm{FR}$, the use of a peripheral reference should account for crystalline lens scatter [56].

4. Negligible macular pigment at a peripheral reference site. If the comparison method is used, the same rules apply as per HFP; provided the peripheral locus is eccentric enough to exclude any macular pigment contribution, the assumption will hold. Choosing a point far enough away from the fovea is easier with FR, since there is little participation required by the subject. Delori et al. [56] commented that the use of a peripheral reference in FR is enough to reduce the influence of the ocular media on the MPOD measurement. Hammond et al. [54], however, argue that regardless of this, as an objective technique FR will suffer from a loss of signal as a result of increased lens scatter and density in some subjects.

5. MPOD measured over the entire stimulus area. Whereas with the psychophysical methods there is some disagreement regarding which part of the macular pigment distribution is actually being measured with the test stimulus, with FR there is a general consensus that the MPOD is the mean amount over the chosen detection field [56, 98, 136, 141]. However, this assumption has not been verified [54].

\section{Validity}

Like HFP, the validity of FR for measurement of MPOD can be assessed by plotting spectral and spatial profiles of macular pigment and comparing these with in vitro data. Van de Kraats et al. [58] argue that any technique demonstrating an increase in MPOD following increased lutein intake is also an indication of its validity. Several reflectometry studies have shown such an increase $[3,136,152]$.

Spectral profiles of macular pigment have not been generated to the extent that they have in HFP studies. In fact, there appears to be only one study that has comprehensively investigated this aspect of validity. Delori et al. [56], used the comparison technique and measured reflectance at wavelengths of $430,450,470,490,520$ and $550 \mathrm{~nm}$. Their results from 147 subjects accurately matched the spectral curve of macular pigment in vitro, albeit with some small systematic deviations such as lower values at $430 \mathrm{~nm}$ (see Figure 12). The 
investigators point out, however, that the deviations altered depending on which in vitro curve they chose for comparison, highlighting the point that the true macular pigment absorption spectrum is not known with enough certainty to assume that the reflectometry deviations are an inaccuracy. It is also interesting to note that lower values at the short wavelength end of the spectrum are also a common finding in HFP spectral profiles (see Figure 4), perhaps lending further support to there being a genuine difference between the in vivo and in vitro macular pigment spectral profile.

The plotting of 'macular pigment maps' to assess macular pigment spatial profiles has become reasonably commonplace in FR studies [e.g. 59, 108, 116, 135, 136, 137]. For SLOs, this generally involves a digital subtraction of the images obtained at two wavelengths (maximal and minimal macular pigment absorption). Chen et al. [108], used a modified fundus camera (the method is described above) and obtained spatial distributions for 54 subjects of various ages. These distributions, divided into three age groups, are shown in Figure 11. The decline in macular pigment from the fovea is rapid and symmetrical, very similar to the decline in macular pigment expected from in vitro knowledge and also from HFP-derived plots of MPOD (see Figure 3).

\section{INSERT FIGURE 11 ABOUT HERE}

Chen et al. [108] looked at the half width of macular pigment distribution (HWMPD) for each of the age groups, and found a significant increase (i.e. widening) with age. With further analyses they also noted that 'shoulders' of varying type were present in the MPOD profile of all subjects. Small irregularities in the otherwise undisturbed decline of macular pigment with eccentricity have been reported in other studies [5, 44, 59, 64, 71, 75, 115-117, 153, 154], both in vivo and in vitro, and are the subject of much ongoing discussion [e.g. 155].

Recently, the MPR [58] has been used to investigate MPOD distribution, or more specifically, to plot the individual distributions of lutein and zeaxanthin [152]. Rather than macular pigment maps, reflection spectra were taken at a variety of eccentricities up to $8^{\circ}$ from the fovea, in a similar manner to HFP.

An indirect way of demonstrating validity is to compare results with those of a technique with established validity, i.e. HFP. This has been done in several FR studies with fairly good results $[56,58,59,141]$.

\section{$\underline{\text { Reliability }}$}


With so many different instruments being used to measure MPOD by $\mathrm{FR}$, there ought to be an abundance of reliability data available. However, few studies have assessed the intersession reliability of their devices, although more have assessed within-session reliability. This is perhaps because, unlike HFP, the actual measurement time in FR is short and does not demand too much effort from the subject; hence it's more convenient to take repeat measurements within the same session. That said, Zagers et al. [146] believed the variability in their intra-session MPOD results was the result of fixation errors, with the less experienced subjects showing greater variability. Nonetheless, as Snodderly and colleagues point out, inter-session reliability is really more valuable than intra-session reliability [50]. Since results generally show higher variability between sessions, this is a more robust test for an instrument. Table 3 outlines the reliability indicators provided in FR studies regarding instrument reliability. It shows that for the studies with published data on reliability, the results are good and comparable with HFP.

\section{INSERT TABLE 3 ABOUT HERE}

\section{Advantages and disadvantages of FR}

Advantages include: 1) as an objective method it requires minimal effort from the subject 2) quick measurement time 3 ) density maps of macular pigment distribution can be plotted quickly 4) reliability appears to be good in several instruments 5) suitability for many subject populations including children. Disadvantages include: 1) pupil dilation normally required 2) the need for precise alignment before measurements 3 ) unpleasant light levels because of the requirement for photopigment bleaching 4 ) the need to control for light scatter, which can include considerable modeling 5 ) costly and complicated instruments, although attempts are being made to produce less expensive reflectometers using commonly available equipment $[59,98,137]$.

\section{Fundus autofluorescence}

One of the newer ways for measuring MPOD in vivo relies on the intrinsic fluorescence, or autofluorescence (AF), of lipofuscin in the retinal pigment epithelium (RPE). Lipofuscin in the RPE is a waste product of photoreceptor outer segment phagocytosis and it accumulates with age [156-158]. When excited with light wavelengths of 400 to $590 \mathrm{~nm}$, lipofuscin fluoresces, emitting light in the wavelength range $520-800 \mathrm{~nm}$ [159]. 
Delori [156] was the first to develop a technique for fundus AF with the primary aim of measuring lipofuscin. Further studies by Delori et al. [157] and von Rückmann et al. [158] provided evidence for lipofuscin being the main fluorophore in AF. It was their observations of a decrease in AF at the macula that lead to the use of $A F$ as a means for measuring MPOD. For an in-depth look at fundus AF and its application, see Schmitz-Valckenberg et al. [160]. The current review will concentrate on the use of AF in macular pigment measurement.

\section{Procedure}

To recall, the absorption spectrum of macular pigment is in the range $400-540 \mathrm{~nm}$ [6] and the absorption spectrum of lipofuscin is in the range $400-590 \mathrm{~nm}$ [159]. Since macular pigment is located anterior to lipofuscin, incoming light directed the fovea will be absorbed by the macular pigment before it reaches the lipofuscin, provided the wavelength of the light is within the absorption range of macular pigment. As a result, there will be an attenuation of lipofuscin fluorescence at the macula; the more macular pigment present, the higher the level of attenuation. By comparing the emitted AF at the fovea and parafovea of two excitation wavelengths, one that is well absorbed by macular pigment and one that is not, MPOD can be calculated [56].

Two AF procedures exist for measurement of macular pigment. The first is a comparison method as used in HFP and some forms of FR. The emitted fluorescence is collected from a foveal and parafoveal sampling area, and then compared to give a measure of MPOD [e.g. 56]. The second and more common procedure is an imaging method whereby up to 32 images $[39,129]$ are taken in succession with one or two wavelengths. The images are aligned (manually or using dedicated software) and averaged, then a greyscale index of intensity is used to generate density maps of macular pigment, which includes a measure of peak MPOD. Key studies using the AF imaging technique include those of Wüstemeyer et al. [25], Berendschot and van Norren [141], Delori et al. [117], Liew et al. [161], Trieschmann et al. [162] and Wolf-Schnurrbusch et al. [154].

\section{$\underline{\text { Instrumentation }}$}

By far the most commonly used instrument for AF acquisition of MPOD is the confocal SLO, purpose-built [e.g. 116, 141] or a modified version of a commercially available SLO [e.g. 25, 161, 163]. All SLOs use the imaging method of fundus AF. The subject fixes a target whilst multiple AF fundus images, usually taken over a $20^{\circ}$ field, are obtained at wavelengths of 
$488 \mathrm{~nm}$ and $514 \mathrm{~nm}$. A barrier filter above or close to the threshold of MP absorption (e.g. $530 \mathrm{~nm}$ ) is used to ensure that the emitted AF is only collected outside the absorption range of macular pigment, thereby avoiding any further absorption and allowing a single-pass measurement rather than a double-pass as used in reflectometry. All the AF images are aligned and averaged for each wavelength. A computer program (see Trieschmann et al. [162] for details) digitally subtracts the averaged images at the two wavelengths and uses a greyscale index of intensity to create a map of MPOD. A foveal MPOD value is calculated at a point eccentricity $[117,161,164]$ or within a certain area centred on the fovea $[25,154$, 161, 163]. As in FR, a correction should be made when using SLOs, to account for the argon laser lines not coinciding exactly with the maximum and minimum wavelengths of macular pigment absorption [26, 39, 141, 151, 153, 164].

Other equipment that has been used to assess MPOD using AF includes the fundus fluorometer/spectrophotometer (first described by Delori [156] and used specifically for macular pigment measurement by Delori et al. [56]) and a modified fundus camera [117, 159]. The fundus fluorometer employs the comparison method. A number of different wavelengths are directed at a $3^{\circ}$ retinal area and the fluorescence is collected from a $2^{\circ}$ sampling field concentric within the $3^{\circ}$ area [56]. The subject is asked to fixate centrally or at $7^{\circ}$ in order to obtain emission data from the fovea and parafovea. MPOD is then calculated using the foveal and parafoveal AF information at excitation wavelengths of $470 \mathrm{~nm}$ and 550 $\mathrm{nm}$. In contrast, the modified fundus camera employs the imaging method. The camera is coupled to a cooled CCD camera and takes pictures of a $15^{\circ}$ retinal field using wavelengths of 470 and $545 \mathrm{~nm}$. At this point the technique becomes very similar to that of SLO AF imaging, i.e. image alignment and analysis by a computer program, thus providing macular pigment density maps, including a measure of peak MPOD [117, 159].

Common to all forms of AF instrumentation is the need for bleaching of the visual pigments prior to measurement (see 'assumptions') and pupil dilation, although a non-mydriatic version has been described [153].

\section{One-wavelength versus two-wavelength AF}

The vast majority of AF-based macular pigment studies have used two wavelengths (corresponding to high and low macular pigment absorption) to derive MPOD. There have been a handful of studies, however, that have used only the high absorption wavelength [19, $39,129]$. It is then presumed that any reduction in AF across the imaged area is due entirely to the presence of macular pigment [19]. Whilst some good correlations between MPOD 
measured with one-wavelength AF and minimum motion photometry have been found [39, 129], certain criticisms have been leveled at the one-wavelength method. Principally, the problem lies with the assumption that the fluorophores, i.e. lipofuscin, are distributed evenly across the imaging field. This is not the case [165] and, as a result, any attenuation in AF could be due to the presence of macular pigment but may also be a consequence of a lower level of lipofuscin in that area $[19,159,162]$. The use of a second, longer wavelength that is minimally absorbed by macular pigment eliminates this issue. Trieschmann et al. [162] compared the one and two-wavelength methods on 120 subjects. They concluded that onewavelength AF is acceptable as a screening method, particularly in view of its widespread availability in SLOs, whilst two-wavelength AF should always be used for precise MPOD assessment. The same conclusion was reached by Sharifzadeh et al. [153] using a CCD camera-based AF device.

\section{Assumptions}

Macular pigment assessment using AF assumes the following:

1. There are no fluorophores anterior to the macular pigment. Delori et al. [56] did find evidence of such a fluorophore and noted that it would cause a small underestimation of MPOD. This underestimation is minimized by detecting AF at a longer wavelength $-710 \mathrm{~nm}$ [56].

2. Lipofuscin at the fovea has the same excitation spectrum as lipofuscin in the surrounding retina. It is unknown whether this is entirely correct but according to Delori and colleagues [56], any differences are not big enough to affect the measured macular pigment spectral curve, as determined by their technique. The total amount of lipofuscin is known to vary across the retina but this is accounted for as long as the two-wavelength method is being used.

3. Any foveal-perifoveal differences in absorbers other than the macular pigment - retinal blood, photoreceptor pigments and RPE melanin - have a negligible effect on the measured MPOD. Delori et al. [56] investigated these assumptions in detail and found that retinal blood differences had virtually no effect and photoreceptor bleaching meant there was very little error in terms of photopigment differences. They did, however, find that RPE melanin slightly overestimated MPOD. 
4. Negligible macular pigment at any peripheral reference site. This is particularly important when the comparison technique is adopted but should be a true assumption because an adequate eccentricity is more easily accomplished with objective than subjective MPOD techniques.

\section{Validity}

The only study to date that has investigated the validity of AF in terms of a spectral comparison with in vitro MPOD data is that by Delori et al. [56]. Excitation wavelengths of 430, 470, 510 and $550 \mathrm{~nm}$ were used on 147 healthy-eyed subjects, plus 450, 490 and 530 $\mathrm{nm}$ on two of these subjects. The resultant spectral profiles for seven subjects are shown in Figure 12, along with the equivalent spectral profiles using a FR technique. The curves are in very good agreement with the chosen in vitro macular pigment curve, attesting to the validity of the AF method of MPOD measurement, in healthy eyes at least.

\section{INSERT FIGURE 12 ABOUT HERE}

Spatial profiles (or maps) of MPOD are the norm for AF imaging. In terms of validity, Robson et al. [39] demonstrated the symmetrical nature of macular pigment distribution (Figure 13), in line with findings using HFP and FR. Also like HFP and FR, however, an array of interindividual macular pigment distributions have been found. Figure 14, for instance, shows two distinct MPOD spatial profiles from a study by Wolf-Schnurrbusch et al. [154]. The top image is the classic distribution of a central peak in macular pigment with a rapid decline as distance from the fovea increases. The bottom image has a central peak in macular pigment followed by a decline, and there is also a secondary peak (a 'parafoveal ring') before further decline. In this particular study, the average eccentricity of the parafoveal ring was $0.66^{\circ}$ from the fovea, which is in line with several other studies [59, 116, 117]. Many researchers now propose that the total complement of macular pigment, rather than the peak amount, may better represent an individual's risk for, or protection from, AMD (see review by Bernstein et al. [155]).

\section{INSERT FIGURES 13 AND 14 ABOUT HERE}

In several studies, AF has been compared with other techniques of MPOD assessment, including HFP, using the same set of subjects [39, 56, 60, 129, 141]. All have shown good correlations, albeit with some systematic differences. 
$\underline{\text { Reliability }}$

As with FR, most tests of reliability concerning AF have been carried out within the same session and are perhaps, therefore, not as useful as inter-session reliability data.

Nevertheless, the results are impressive, indicating similar, if not better, reliability than HFP and FR. Table 4 contains a list of AF studies that have provided information on reliability.

\section{INSERT TABLE 4 ABOUT HERE}

\section{Advantages and disadvantages of $\mathrm{AF}$}

Advantages include: 1) its objectivity; as an objective test, AF requires no subject participation other than a short period of reasonable fixation 2) quick measurement time 3) spatial plots of macular pigment distribution produced as standard in AF imaging 4) good test-retest reliability 5) applicability to many subject populations, including children. Disadvantages include: 1) the need for pupil dilation 2) equipment expense 3) a lack of commercially-available two-wavelength SLOs 4) the need for photopigment bleaching and therefore unpleasant light levels 5) difficulty obtaining clear images from eyes with lens opacities.

\section{Resonance Raman spectroscopy}

With the exception of electrophysiology methods, resonance Raman spectroscopy (RRS) is the most recently developed MPOD technique and, arguably, the most controversial. First described by Bernstein et al. in 1998 [166], RRS takes advantage of lutein and zeaxanthin's ability to exhibit a phenomenon called Raman scattering [167]. Over the last ten years the use of RRS to measure MPOD has quickly gained momentum, with many papers published on its use [57, 111, 168-178].

\section{Procedure}

When monochromatic light is directed at a molecule, some of the light is scattered. Most of the light is scattered elastically (Rayleigh scattering) but a small proportion is scattered inelastically (Raman scattering). When this inelastic back-scattering happens, there is a wavelength shift of the incident light, known as a Raman shift; the shift in wavelength is molecule-specific and therefore the back-scattered light can be collected and analyzed to identify the molecule in question. Usually the Raman signal is very weak and as such is not 
easily identified. However, if the incident wavelength overlaps with the absorption spectrum of the molecule, a large resonance enhancement of the Raman scattered light occurs and the molecule can be recognized. Carotenoids, including lutein and zeaxanthin, are an excellent example of this. When excited by $488 \mathrm{~nm}$ argon laser light, they exhibit a resonance enhancement of up to five orders of magnitude [169], with three characteristic Raman spectral peaks [166, 167, 171], as shown in Figure 15. The strongest peak is at 1525 $\mathrm{cm}^{-1}$ and this is the Raman line that is subsequently quantified in Raman counts (RCs).

RRS is completely different from almost all other MPOD techniques in that it measures absolute levels of macular pigment in a $1 \mathrm{~mm}\left(3.5^{\circ}\right)$ area, with no peripheral consideration at all. The researchers in this field claim that this is acceptable since the signal is derived directly from the pigment itself, rather than relying on light that must travel to deeper layers of the retina $[111,171]$ and that furthermore, the signal is only strong enough to register at carotenoid concentrations found in the macula, rather than in any other structures such as the cornea and lens [166].

\section{INSERT FIGURE 15 ABOUT HERE}

\section{$\underline{\text { Instrumentation }}$}

The setup for RRS detection of MPOD is shown in Figure 16. It consists of a $488 \mathrm{~nm}$ argon laser that is directed at the fovea. The returning back-scattered light is filtered so that only Raman shifted light is sent to the Raman spectrograph (via a fibre optic bundle). The spectrograph is linked to a CCD camera, which is in turn linked to a computer that is programmed to subtract the background fluorescence and quantify the intensity of the Raman peaks, in particular the $1525 \mathrm{~cm}^{-1}$ line. Optical alignment of the instrument to the fovea is achieved in human subjects by use of a red LED and a small portion of the blue argon laser light. The red LED is visible to the observer as a polka-dot pattern and the laser light as a blue disc. By small head movements along a head rest, the subject lines up the two images, at which point the operator pushes a button to begin the whole procedure. The $488 \mathrm{~nm}$ light is directed as a $0.5 \mathrm{~mW}, 1 \mathrm{~mm}$ spot onto the macula for 0.5 seconds [170, 171, 173]. Later studies using RRS have altered the settings slightly by using a $1 \mathrm{~mW}, 1 \mathrm{~mm}$ spot directed at the macula for 0.25 seconds $[57,174,175,179]$. Five measurements are taken at intervals of $30-180$ secs (the time is dependent on afterimage fading) and the original protocol [171] dictated that the three highest recordings are used in the data analysis, to allow for subjects that blink or misalign. 
Prior to measurement, pupil dilation to at least $6 \mathrm{~mm}$ is necessary [170, 171]; some later studies have found that dilation to at least $7 \mathrm{~mm}$ gives more reliable results $[57,174]$ - see discussion about pupil size in the 'validity' section.

\section{INSERT FIGURE 16 ABOUT HERE}

RESONANCE RAMAN IMAGING

One limitation of RRS in its classic form is that it is limited to measuring MPOD in a $1 \mathrm{~mm}$ $\left(3.5^{\circ}\right)$ area centred on the fovea, rather than being able to produce a spatial profile of macular pigment across a larger retinal area. The technique has therefore been extended into an imaging mode covering a $3.5 \mathrm{~mm}$ area; initially using excised donor eyes [172], then more recently, following further modification, using living volunteers $[175,178]$.

\section{Assumptions}

The assumptions associated with RRS are listed below. They are elaborated upon in the following section on validity.

1. Accurate alignment and fixation by the subject, and no significant head movement during image capture.

2. No significant effect on the Raman signal by differing levels of crystalline lens yellowing, i.e. absorption, within and across age groups.

3. No significant effect on the Raman signal by inter-individual differences in the level of lens diffusion (scatter and aberration).

\section{Validity}

The validity of RRS has been a subject of fierce debate in the literature [e.g. 54, 180, 181 186]. It is beyond the scope of this review to repeat the issues of contention verbatim; rather, the main points are summarized.

The research group that developed RRS for measurement of MPOD have shown that it is very specific and sensitive to measurement of lutein and zeaxanthin [166, 170, 171]. However, unlike the other methods of MPOD measurement, RRS cannot generate spectral absorption curves of macular pigment to be compared with in vitro curves. Instead, the 
investigators attest to the validity of RRS in several other ways. Firstly, the strength of the Raman signal from donor maculae has been compared with actual macular pigment levels as measured by HPLC (Figure 17) [166]. The results revealed a highly linear correlation $(r=$ 0.94, no $p$-value provided). Secondly, a model eye containing known amounts of lutein was measured with the Raman device (Figure 18) and the results again demonstrated a linear correlation ( $r=0.99$, no $p$-value provided) [171]. Thirdly, the Raman signals from six intact monkey eyes were determined then compared with their HPLC-measured macular pigment level (Figure 19) [171]. The correlation between the two was good but not perfect $(r=0.68$, no $p$-value provided); the authors attributed this to differences in the detection area between the Raman (1 mm) and the HPLC (5 mm) method, and to difficulties with foveal alignment. Finally, Bernstein et al. [170] found a perfect linear correlation between Raman signals from lutein and zeaxanthin solutions placed in a model eye and their known concentrations (Figure 20A), up to about 0.35 density units (equivalent in this case to $1600 \mathrm{RCs}$ ). At higher concentrations, the Raman response became saturated and therefore non-linear (Figure 20B), as a result of the $488 \mathrm{~nm}$ laser beam being unable to penetrate increasingly dense carotenoid concentrations [170]. Ermakov et al. [174] found a similar linear then non-linear response for various zeaxanthin concentrations, again using a model eye.

\section{INSERT FIGURES 17-20 ABOUT HERE}

Those questioning the validity of RRS as a method of MPOD measurement have made several criticisms of the above calibration procedures. The issues of contention include whether or not the model eye is a true representation of a real in vivo eye; whether or not the increasing underestimation of MPOD at higher concentrations is a problem in ordinary subject populations; and the credibility of using the monkey data as evidence of validity, given that the level of RCs was in general far higher than most human levels and well above the point at which the plateau occurs in the external calibration curve (see Figures 19 and 20).

Studies of MPOD using RRS have consistently shown a strong decline in macular pigment with increasing age (see, for example, Figure 21). Hammond et al. [54] argue that this is another area where the validity of RRS is questionable, since with all other MPOD techniques, there appears to be little or no age-related macular pigment decline [141]. The developers of RRS believe that the decrease is genuine [110,111, 169-171, 173-175] and not just attributable to increasing lens absorption (yellowing) and diffusion (scatter and aberration) with age, as suggested by Hammond and Wooten [54, 182-184]. An independent study simulating incremental increases in lens yellowing and scatter found that the Raman 
signal intensity was significantly attenuated as the density of the yellow and scatter filters increased [179]. The authors concluded that when using RRS to assess MPOD, the status of the lens needs to be taken into account. In other words, the large decline in MPOD with age reported in many RRS studies is unlikely to represent a true drop in macular pigment levels and therefore the validity of RRS in older subjects, at least, is uncertain.

\section{INSERT FIGURE 21 ABOUT HERE}

Another reason for the decrease in MPOD with age - as found by RRS - may be due in part to inadequate pupil dilation. Studies have shown that the Raman signal is weakened when pupil diameter is smaller than $7 \mathrm{~mm}[57,171,174]$. This is because the entrance/exit pupil of the Raman instrument is also $7 \mathrm{~mm}$ and thus any pupil diameter less than this will result in a loss of signal [171, 174]. Neelam et al. [57] found that the significant age-related decline in RRS-derived MPOD of their subject population was reduced to a non-significant level when subjects with inadequate pupil dilation $(<7 \mathrm{~mm})$ were excluded. It would therefore seem that an inability to sufficiently dilate the pupils of some older individuals might contribute to the decline in MPOD seen with RRS. Further to this, small head movements in subjects whose pupil diameter is at the $7 \mathrm{~mm}$ limit could also reduce the Raman signal, regardless of age $[54,176,182-184,186]$, although Bernstein and colleagues may contend that their procedure of taking the three highest RCs of five measurements allows for such head movements.

Widely varying macular pigment spatial profiles have been observed using the RR imaging (RRI) device in living human subjects, including asymmetries and local depletions [178]. Although such distributions are not normally typical of other MPOD techniques, in comparing the integrated macular pigment densities of the entire imaged area with the densities measured over the same area with an AF method, the investigators found a very high correlation in 17 subjects ( $r=0.89$, no $p$-value provided). Furthermore, the integrated macular pigment levels of 11 donor maculae measured with the RRI instrument were compared with the levels as measured by HPLC. The correlation between the two was very strong $(r=0.92, p<0.0001)$, although the influence of the ocular media was removed and therefore may have lead to an erroneously high agreement. Nevertheless, the validity of RRI looks promising.

Indirect methods of assessing validity, mentioned previously, consist of comparisons with more established, validated techniques, and the ability to show rises in MPOD with increased lutein and/or zeaxanthin intake. The latter has apparently been proven, although 
the study was only briefly described [110]. RRS-measured MPOD has been compared with HFP-measured MPOD in only two, detailed published papers [57, 176]. Neelam et al. [57], using Bland-Altman plots, demonstrated an agreement between the two techniques close to statistical significance. The correlation, as described by Pearson product-moment correlation coefficient, was $r=0.32$ (derived by Hammond and Wooten [184]). Although statistically significant, this correlation only explains $10 \%$ of the variance in the two methods [184]. Hogg et al. [176] also found a weak, albeit statistically significant, correlation between RRS and HFP ( $r=0.26, p=0.012)$. They did not feel it was good enough for the two techniques to be interchangeable. Bernstein et al. [110] reported a better correlation $(r=0.467, p=0.0024)$ in 40 healthy subjects, but their study was not described in any detail.

\section{$\underline{\text { Reliability }}$}

RRS appears to exhibit good within- and between-session reliability in the majority of studies (Table 5). The high variation in readings experienced in subjects from a study by Obana et al. [177], particularly in individuals with age-related maculopathy (ARM), is a notable exception. This is the only study that has provided any RRS reliability data for subjects with ocular disease and is an indication, perhaps, that more data is needed before evaluating information on MPOD in these populations. That said, 32 out of the 180 eyes with ARM had worse visual acuity than the recommended limit for RRS of $6 / 24(20 / 80)$. This may have contributed to the variation. In view of the variation, Obana and colleagues chose to accept only the highest of the five Raman readings in all their subjects [177]. This means that $80 \%$ of the measurements were rejected, which seems a lot for a technique claiming to be validated. Nevertheless, many authorities have shown that MPOD measurements, as derived by RRS, do have good test-retest reliability (see Table 5).

\section{INSERT TABLE 5 ABOUT HERE}

\section{Advantages and disadvantages of RRS}

Advantages include: 1) sensitivity and specificity for retinal carotenoids 2) rapid measurements requiring only momentary fixation from the subject 3 ) the possibility of quickly generated, detailed spatial distribution plots of macular pigment, using the RRI method 4) reasonable reliability 5) measurements possible in many individuals, including those with reduced acuity, up to 6/24 (20/80). Disadvantages include: 1) the need for pupil dilation 2) the reliance on subjects for accurate alignment, i.e. the lack of an objective alignment procedure 3) afterimages between measurements 3) questionable validity 4) attenuation of 
the Raman signal with changes in the ocular media and inadequate pupil dilation 5) instrumentation that is highly specialized and expensive 6) RCs not being readily convertible to macular pigment density units, making direct comparisons with other techniques difficult.

\section{Electrophysiology - visual evoked potentials}

The first suggestion that visual evoked potentials (VEPs) could potentially be used to detect macular pigment was made over ten years ago, by Moreland et al. [37]. This was investigated further by Robson et al. [92] some time later. However, it is only very recently that this particular technique has looked like it could be a truly viable method for measuring MPOD. Using steady-state VEPs, Robson and Parry [41] measured MPOD across a range of eccentricities in three subjects. Blue-green gratings on a colour monitor were employed and these same gratings were also used to measure MPOD with HFP (see 'recent developments' in HFP section). The VEP and HFP results were compared with each other as well as with the equivalent MPOD as measured by minimum motion photometry. This required a correction factor on the part of the VEP and HFP results, to allow for the overlapping phosphor emissions of the blue and green stimuli. The correlation between all three techniques was excellent $(r \geq 0.94, p<0.0005$, in all cases), suggesting that steadystate VEPs have potential as a valid, objective method for measuring macular pigment and its distribution.

\section{DISCUSSION}

There are currently two main psychophysical techniques for measuring MPOD in vivo, and three main objective techniques. All take advantage of the spectral absorption properties of macular pigment but in very diverse ways. This diversity may be useful for macular pigment research but it does present difficulties for those wishing to compare MPOD values between techniques. For instance, does the value represent the peak density of macular pigment, the density of macular pigment at a certain point within the fovea, or the total amount within the target area?

If macular pigment measurement is to become commonplace in large populations, then equipment investors will have an important decision to make with regards to the method they choose to employ. Unfortunately, as each MPOD technique has its own benefits and limitations, there is no clear ideal choice, as highlighted by Beatty, van Kuijk and Chakravarthy [187]. Heterochromatic flicker photometry is probably the most affordable choice. It is also an established, valid and reliable method, particularly when protocols are 
followed as per 'customized' HFP [50, 52]. There is, however, the problem that some individuals find this task very difficult and their results cannot necessarily be relied upon. $A$ commercially available objective technique would therefore be desirable, possibly through adaptation of a scanning laser ophthalmoscope or fundus camera. The former is often used in a hospital setting and the latter is commonly found in optometric practice. A future objective technique could make use of FR or AF to assess MPOD, although AF may be preferred over FR because it is less influenced by light scatter and appears to have better reliability. Both have the facility to measure the spatial distribution of macular pigment and this seems to be an increasingly useful advantage $[8,39,74,108,116]$. Bhosale, Zhao and Bernstein [112] observed elevated lutein levels at the macula and at the peripheral retina in donors known to be using high dose lutein supplements. Therefore, if non-macula areas are not taken into account, the total complement of macular pigment may be underestimated, particularly with methods such as HFP, where the eccentric references are assumed to have virtually no macular pigment. The main issue associated with macular pigment screening using an objective technique is the need for pupil dilation, although several non-mydriatic devices have now been developed [58, 59, 153].

It is our view that the measurement of MPOD is best conducted using an objective technique based on FR or AF but we acknowledge that a commercial instrument capable of this is not currently available. The development of such an instrument will aid research in this area and provide a better understanding of the relationship between MPOD and AMD, as well as supporting MPOD screening in a clinical setting. 


\section{Figure and table legends}

Figure 1. Schematic diagram of a Maxwellian view optical system, as used by Wooten et al. [46]. A1-A3 = apertures 1-3. BF = blocking filter (removes stray light). BS1 and BS2 = beam splitters 1 and 2. $\mathrm{C}$ = flicker vanes with a first surface mirror (produces alternation of the test and reference lights). HM1-HM3 = hot mirrors 1-3 (reduce heat transfer). IF1 and IF2 = interference filters 1 and 2 . L1-L17 = lenses 1-17 (achromatic, planoconvex). $M=$ monochromator (produces the test wavelength). M1-M4 = mirrors 1-4 (right angle, first surface). ND = neutral density filter (together with interference filters, produces the reference and background wavelengths). $\mathrm{R}=$ reticle. $\mathrm{S}=$ source light, in this case a xenon arc lamp. $\mathrm{W}$ = wedge (used to adjust the radiance of the test light). [Reprinted from Wooten et al. [46], with permission from the Association for Research in Vision and Ophthalmology.]

Figure 2. Schematic diagram of a free view optical system, as used by Wooten et al. [46]. $\mathrm{A} 1$ and $\mathrm{A} 2=$ apertures 1 and $2 . \mathrm{BS}=$ beam splitter. D1 and D2 = optical diffusers (increase transmission efficiency). $\mathrm{H}$ = hole (1 inch circular viewing hole). $\mathrm{L} 1$ and $\mathrm{L} 2=$ lenses 1 and 2 (achromatic, planoconvex). PC = photocell. $S 1$ and $S 2=$ source lights 1 and $2(3 \times 470 \mathrm{~nm}$ LEDs for S1, i.e. background field, and $2 \times 458 \mathrm{~nm}$ plus $1 \times 570 \mathrm{~nm}$ for S2, i.e. test field). [Reprinted from Wooten et al. [46], with permission from the Association for Research in Vision and Ophthalmology.]

Figure 3. A detailed spatial profile of one subject's macular pigment density, obtained using heterochromatic flicker photometry. Here, measurements have been taken along the horizontal (filled squares) and vertical (open circles) meridians of the retina, demonstrating a rapid and symmetrical decline in macular pigment with increasing eccentricity [Reprinted from Hammond et al. [44], with permission from the Optical Society of America.]

Figure 4. The relative macular pigment spectral absorption profile of one subject (filled squares), as derived by heterochromatic flicker photometry. The continuous curve is an in vitro combination template as described in the text. Small deviations from the template at wavelengths below about $440 \mathrm{~nm}$ are typical. The reason for this is not clear and several theories have been proposed [see, for example, 54, 66, 71]. However, above $440 \mathrm{~nm}$, the in vivo and in vitro methods are in very close agreement, so measurements of peak macular pigment optical density should remain accurate. [Reprinted from Wooten and Hammond [71], with permission from the American Academy of Optometry.] 
Figure 5. A high correlation $(r=0.91, p<0.00001)$ between macular pigment optical density measured with a centrally positioned $1^{\circ}$ test stimulus and a $12^{\prime}$ test stimulus positioned $0.5^{\circ}$ from the central fovea lends support to the edge hypothesis of heterochromatic flicker photometry. [Reprinted from Hammond et al. [44], with permission from the Optical Society of America.]

Figure 6. Left panel: Macular pigment distribution in the central $1.5^{\circ}$, as determined by Bone et al. [68], using heterochromatic flicker photometry. The horizontal line is the average macular pigment optical density (MPOD) for 10 subjects, calculated using a centrally positioned $1.5^{\circ}$ circular test stimulus (grey area $= \pm 1$ standard deviation). The filled circles depict the average MPOD at various eccentricities (bars $= \pm 1$ standard deviation), determined using a number of annular stimuli with central fixation marks (right panel). Whereas Hammond et al. [44] used a very small stimulus placed at the required retinal eccentricity, Bone and colleagues have used annular stimuli. This is an alternative method of knowingly measuring MPOD at a retinal eccentricity equivalent to the stimuli radii. The intersection point at $0.38^{\circ}$ is the position at which MPOD appears to be measured when using a $1.5^{\circ}$ test stimulus, i.e. $51 \%$ of the stimulus radius. Right panel: The four annular stimuli and two circular stimuli ( $1.17^{\circ}$ circular stimulus not used in this graph). ID $=$ inner diameter, OD = outer diameter. [Reprinted from Bone et al. [68], with permission from Elsevier.]

Figure 7. Test configuration used in minimum motion photometry (left panel), with the associated stimuli dimensions given in the table (right panel). [Reprinted from Robson et al. [129], with permission from Pion Limited.]

Figure 8. Apparent motion photometry. Left panel: Apparent movement to the right when the red bars are brighter than the blue, and vice-versa. Right panel: The test configuration for a parafoveal target [130].

Figure 9. Schematic diagram of the Foveal Reflection Analyzer 1, from Zagers et al. [146]. In (a): $F=$ spectral filters. Lamp $=30 \mathrm{~W}$ halogen lamp. $L 1-L 11=$ lenses $1-11 . L f=$ front lens. $\mathrm{Li}=$ insertable lens. $\mathrm{Mh}=$ mirror with central hole. $\mathrm{Mi}=$ insertable mirror. $\mathrm{P}=$ pupil plane. $\mathrm{R}=$ retinal plane. $\mathrm{P}^{\prime}$ and $\mathrm{R}^{\prime}$ = planes conjugate to $\mathrm{P}$ and $\mathrm{R}$. V = video camera. $\ln (\mathrm{b})$ : The dilated pupil with entrance and exit pupil shown to scale (left panel). The illuminated field and the concentric sampled field, with fixation cross hairs (right panel). [Reprinted from Zagers et al. [146], with permission from the Optical Society of America.] 
Figure 10. Schematic diagram of the Macular Pigment Reflectometer, from van de Kraats et al. [58]. L1-L8 = lenses 1-8. M = mirror. [Reprinted from van de Kraats et al. [58], with permission from SPIE.]

Figure 11. Spatial profiles of macular pigment density in three age groups, obtained by fundus reflectometry: (a) young (24.8 \pm 2.6 years), (b) mid-age (40.2 \pm 8.3 ) and (c) old (67.5 \pm 7.1). [Reprinted from Chen et al. [108], with permission from Informa Healthcare.]

Figure 12. Left panel: Log ratio autofluorescence (AF) plotted against wavelength for seven subjects (symbols) along with the scaled macular pigment spectra (curves). The age of each subject is given to the left of each curve and the derived macular pigment optical density (with $r^{2}$ values of the fits) is given to the right. Right panel: The equivalent fundus reflectometry ('RE') results. [Reprinted from Delori et al. [56], with permission from the Optical Society of America.]

Figure 13. Autofluorescence images (first and third columns) and macular pigment spatial profiles (second and fourth columns) for 8 subjects $(A-H)$. Open circles indicate the vertical meridian and filled circles the horizontal meridian. The arrows indicate disruptions due to prominent blood vessels. [Reprinted from Robson et al. [39], with permission from Elsevier.]

Figure 14. Two example macular pigment spatial profiles with their corresponding autofluorescence images: (a) classic profile - central peak in macular pigment followed by a rapid decline, and (b) parafoveal ring profile - central peak in macular pigment, plus a secondary peak, before further decline. [Reprinted from Wolf-Schnurrbusch et al. [154], with permission from the Association for Research in Vision and Ophthalmology.]

Figure 15. In vivo Raman spectra of a healthy subject, showing the characteristic peaks of macular pigment. Top trace $=$ before subtraction of the background ocular fluorescence. Bottom trace $=$ after subtraction. [Reprinted from Gellermann et al. [171], with permission from the Optical Society of America.]

Figure 16. Schematic diagram of a resonance Raman spectroscopy macular pigment detector. $\mathrm{Ar}^{+}$laser $=$air-cooled argon laser. $\mathrm{BS}=$ dichroic beam splitter. $\mathrm{F}=$ filter. $\mathrm{LED}=$ light emitting diode. $\mathrm{L} 1-4$ = lenses $1-4 . \mathrm{M}=$ Mirror. NF = holographic rejection notch filter. $\mathrm{TB}=$ trigger button. VHTF = volume holographic transmission grating. [Reprinted from Gellermann et al. [171], with permission from the Optical Society of America.] 
Figure 17. Macular pigment measurements in seven human donor maculae, measured by high-performance liquid chromatography and resonance Raman spectroscopy. [Reprinted from Bernstein et al. [166], with permission from the Association for Research in Vision and Ophthalmology.]

Figure 18. Macular pigment measurements by resonance Raman spectroscopy of four known lutein concentrations placed in a model eye. [Reprinted from Gellermann et al. [171], with permission from the Optical Society of America.]

Figure 19. Macular pigment measurements in six monkey eyes, measured by highperformance liquid chromatography and resonance Raman spectroscopy. [Reprinted from Gellermann et al. [171], with permission from the Optical Society of America.]

Figure 20. Macular pigment measurements by resonance Raman spectroscopy of known lutein and zeaxanthin concentrations placed in a model eye: $A$ = linear response range (filled circles are lutein, open circles are zeaxanthin). $B=$ linear and non-linear response range. [Reprinted from Bernstein et al. [170], with permission from Elsevier.]

Figure 21. The decline in macular pigment optical density with age, as measured by resonance Raman spectroscopy. [Reprinted from Bernstein et al. [170], with permission from Elsevier.]

Table 1. A summary of the main differences between Maxwellian and free view optical systems.

Table 2. A list of all heterochromatic flicker photometry (HFP) studies to date that have given statistical data on test-retest reliability.

a Snodderly et al. [50] also calculated the equivalent values for the left eye, which were $r=$ $0.86,22 \%$ test-retest difference and a coefficient of repeatability of 0.21 .

$\mathrm{ARM}=$ age-related maculopathy, the general term for degeneration of the macular region of the retina, which is normally separated into the two categories of early and late ARM, with the latter also being called age-related macular degeneration [188].

MPOD = macular pigment optical density.

$\mathrm{RE}=$ right eye.

$\mathrm{RPE}=$ retinal pigment epithelium. 
Table 3. A list of all fundus reflectometry (FR) studies to date that have given statistical data on reliability.

$\mathrm{ARM}=$ age-related maculopathy.

FRA = Foveal Reflection Analyzer, a purpose-built reflectometer.

MPR = Macular Pigment Reflectometer, a purpose-built reflectometer.

SLO = scanning laser ophthalmoscope.

Table 4. A list of all autofluorescence (AF) studies to date that have given statistical data on reliability.

AMD = age-related macular degeneration .

MPOD = macular pigment optical density.

Table 5. A list of all resonance Raman spectroscopy (RRS) studies to date that have given statistical data on reliability.

$\mathrm{ARM}=$ age-related maculopathy, the general term for degeneration of the macular region of the retina, which is normally separated into the two categories of early and late ARM, with the latter also being called age-related macular degeneration (AMD) [188].

MPOD = macular pigment optical density 


\section{REFERENCES}

1. Bone RA, Landrum JT, Hime GW, Cains A, Zamor J (1993) Stereochemistry of the human macular carotenoids. Invest Ophthalmol Vis Sci 34: 2033-2040

2. Malinow MR, Feeneyburns L, Peterson LH, Klein ML, Neuringer M (1980) Diet-related macular anomalies in monkeys. Invest Ophthalmol Vis Sci 19: 857-863

3. Neuringer M, Sandstrom MM, Johnson EJ, Snodderly DM (2004) Nutritional manipulation of primate retinas, I: effects of lutein or zeaxanthin supplements on serum and macular pigment in xanthophyll-free rhesus monkeys. Invest Ophthalmol Vis Sci 45: 3234-3243 4. Sommerburg O, Keunen JEE, Bird AC, van Kuijk F (1998) Fruits and vegetables that are sources for lutein and zeaxanthin: the macular pigment in human eyes. Br J Ophthalmol 82: 907-910

5. Snodderly DM, Auran JD, Delori FC (1984) The macular pigment .II. Spatial distribution in primate retinas. Invest Ophthalmol Vis Sci 25: 674-685

6. Snodderly DM, Brown PK, Delori FC, Auran JD (1984) The macular pigment .I. Absorbance spectra, localization, and discrimination from other yellow pigments in primate retinas. Invest Ophthalmol Vis Sci 25: 660-673

7. Bone RA, Landrum JT, Fernandez L, Tarsis SL (1988) Analysis of the macular pigment by HPLC - retinal distribution and age study. Invest Ophthalmol Vis Sci 29: 843-849

8. Trieschmann M, van Kuijk F, Alexander R, Hermans $P$, Luthert $P$, Bird AC, Pauleikhoff D (2008) Macular pigment in the human retina: histological evaluation of localization and distribution. Eye 22: 132-137

9. Bone RA, Landrum JT, Cains A (1992) Optical density spectra of the macular pigment in vivo and in vitro. Vision Res 32: 105-110

10. Ham WT, Mueller HA, Sliney DH (1976) Retinal sensitivity to damage from short wavelength light. Nature 260: 153-155

11. Hammond BR, Caruso-Avery M (2000) Macular pigment optical density in a southwestern sample. Invest Ophthalmol Vis Sci 41: 1492-1497

12. Landrum JT, Bone RA (2001) Lutein, zeaxanthin, and the macular pigment. Arch Biochem Biophys 385: 28-40

13. Khachik F, Bernstein PS, Garland DL (1997) Identification of lutein and zeaxanthin oxidation products in human and monkey retinas. Invest Ophthalmol Vis Sci 38: 1802-1811 14. Leibowitz HM, Krueger DE, Maunder LR, Milton RC, Kini MM, Kahn HA, Nickerson RJ, Pool J, Colton TL, Ganley JP, Loewenstein JI, Dawber TR (1980) The Framingham Eye Study monograph: an ophthalmological and epidemiological study of cataract, glaucoma, diabetic retinopathy, macular degeneration, and visual acuity in a general population of 2631 adults, 1973-1975. Surv Ophthalmol 24: 335-610

15. Klein R, Klein BEK, Linton KLP (1992) Prevalence of age-related maculopathy - the Beaver Dam Eye Study. Ophthalmology 99: 933-943

16. Mitchell P, Smith W, Attebo K, Wang JJ (1995) Prevalence of age-related maculopathy in Australia - the Blue Mountains Eye Study. Ophthalmology 102: 1450-1460

17. Beatty S, Murray IJ, Henson DB, Carden D, Koh HH, Boulton ME (2001) Macular pigment and risk for age-related macular degeneration in subjects from a Northern European population. Invest Ophthalmol Vis Sci 42: 439-446

18. Bone RA, Landrum JT, Mayne ST, Gomez CM, Tibor SE, Twaroska EE (2001) Macular pigment in donor eyes with and without AMD: A case-control study. Invest Ophthalmol Vis Sci 42: $235-240$

19. Trieschmann M, Spital G, Lommatzsch A, van Kuijk E, Fitzke F, Bird AC, Pauleikhoff D (2003) Macular pigment: quantitative analysis on autofluorescence images. Graefes Arch Clin Exp Ophthalmol 241: 1006-1012

20. Ciulla TA, Hammond BR (2004) Macular pigment density and aging, assessed in the normal elderly and those with cataracts and age-related macular degeneration. Am J Ophthalmol 138: 582-587 
21. LaRowe TL, Mares JA, Snodderly DM, Klein ML, Wooten BR, Chappell R, Grp CMPS (2008) Macular pigment density and age-related maculopathy in the carotenoids in agerelated eye disease study - an ancillary study of the women's health initiative.

Ophthalmology 115: 876-883

22. Bone RA, Landrum JT, Tarsis SL (1985) Preliminary identification of the human macular pigment. Vision Res 25: 1531-1535

23. Handelman GJ, Dratz EA, Reay CC, Vankuijk F (1988) Carotenoids in the human macula and whole retina. Invest Ophthalmol Vis Sci 29: 850-855

24. Handelman GJ, Snodderly DM, Krinsky NI, Russett MD, Adler AJ (1991) Biological control of primate macular pigment - biochemical and densitometric studies. Invest Ophthalmol Vis Sci 32: 257-267

25. Wüstemeyer H, Moessner A, Jahn C, Wolf S (2003) Macular pigment density in healthy subjects quantified with a modified confocal scanning laser ophthalmoscope. Graefes Arch Clin Exp Ophthalmol 241: 647-651

26. Trieschmann M, Beatty S, Nolan JM, Hense HW, Heimes B, Austermann U, Fobker M, Pauleikhoff D (2007) Changes in macular pigment optical density and serum concentrations of its constituent carotenoids following supplemental lutein and zeaxanthin: the LUNA study. Exp Eye Res 84: 718-728

27. Brown PK, Wald G (1963) Visual pigments in human and monkey retinas. Nature 200: 37-43

28. Bone RA, Sparrock JM (1971) Comparison of macular pigment densities in human eyes. Vision Res 11: 1057-1064

29. Stabell U, Stabell B (1980) Variation in density of macular pigmentation and in shortwave cone sensitivity with eccentricity. J Opt Soc Am 70: 706-711

30. Pease PL, Adams AJ (1983) Macular pigment difference spectrum from sensitivity measures of a single cone mechanism. Am J Optom Physiol Opt 60: 667-672

31. Pease PL, Adams AJ, Nuccio E (1987) Optical density of human macular pigment. Vision Res 27: 705-710

32. Ruddock KH (1963) Evidence for macular pigmentation from colour matching data.

Vision Res 61: 417-429

33. Moreland JD, Bhatt P (1984) Retinal distribution of macular pigment. Doc Ophthalmol Proc Ser 39: 127-132

34. Moreland JD, Alexander EC (1997) Effect of macular pigment on colour matching with field sizes in the $1^{\circ}$ to $10^{\circ}$ range. Doc Ophthalmol Proc Ser 59: 363-368

35. Davies NP, Morland AB (2002) Color matching in diabetes: optical density of the crystalline lens and macular pigments. Invest Ophthalmol Vis Sci 43: 281-289

36. Bone RA (1980) The role of the macular pigment in the detection of polarized light. Vision Res 20: 213-220

37. Moreland JD, Robson AG, Soto-Leon N, Kulikowski JJ (1998) Macular pigment and the colour-specificity of visual evoked potentials. Vision Res 38: 3241-3245

38. Moreland JD, Robson AG, Kulikowski JJ (2001) Macular pigment assessment using a colour monitor. Color Res Appl 26: S261-S263

39. Robson AG, Moreland JD, Pauleikhoff D, Morrissey T, Holder GE, Fitzke FW, Bird AC, van Kuijk $F$ (2003) Macular pigment density and distribution: comparison of fundus autofluorescence with minimum motion photometry. Vision Res 43: 1765-1775

40. Moreland JD (2004) Macular pigment assessment by motion photometry. Arch Biochem Biophys 430: 143-148

41. Robson AG, Parry NRA (2008) Measurement of macular pigment optical density and distribution using the steady-state visual evoked potential. Vis Neurosci 25: 575-583 42. Werner JS, Donnelly SK, Kliegl R (1987) Aging and human macular pigment density. Appended with translations from the work of MaxSchultze and Ewald Hering. Vision Res 27: 257-268

43. Hammond BR, Fuld K (1992) Interocular differences in macular pigment density. Invest Ophthalmol Vis Sci 33: 350-355 
44. Hammond BR, Wooten BR, Snodderly DM (1997) Individual variations in the spatial profile of human macular pigment. J Opt Soc Am A Opt Image Sci Vis 14: 1187-1196 45. Landrum JT, Bone RA, Joa H, Kilburn MD, Moore LL, Sprague KE (1997) A one year study of the macular pigment: the effect of 140 days of a lutein supplement. Exp Eye Res 65: $57-62$

46. Wooten BR, Hammond BR, Land RI, Snodderly DM (1999) A practical method for measuring macular pigment optical density. Invest Ophthalmol Vis Sci 40: 2481-2489 47. Beatty S, Koh HH, Carden D, Murray IJ (2000) Macular pigment optical density measurement: a novel compact instrument. Ophthalmic Physiol Opt 20: 105-111 48. Mellerio J, Ahmadi-Lari S, van Kuijk F, Pauleikhoff D, Bird A, Marshall J (2002) A portable instrument for measuring macular pigment with central fixation. Curr Eye Res 25: 37-47

49. Bone RA, Landrum JT (2004) Heterochromatic flicker photometry. Arch Biochem Biophys 430: 137-142

50. Snodderly DM, Mares JA, Wooten BR, Oxton L, Gruber M, Ficek T (2004) Macular pigment measurement by heterochromatic flicker photometry in older subjects: the carotenoids and age-related eye disease study. Invest Ophthalmol Vis Sci 45: 531-538 51. Iannaccone A, Mura M, Gallaher KT, Johnson EJ, Todd WA, Kenyon E, Harris TL, Harris T, Satterfield S, Johnson KC, Kritchevsky SB, Study HA (2007) Macular pigment optical density in the elderly: findings in a large biracial midsouth population sample. Invest Ophthalmol Vis Sci 48: 1458-1465

52. Stringham JM, Hammond BR, Nolan JM, Wooten BR, Mammen A, Smollon W, Snodderly DM (2008) The utility of using customized heterochromatic flicker photometry (cHFP) to measure macular pigment in patients with age-related macular degeneration. Exp Eye Res 87: 445-453

53. van der Veen RL, Berendschot TT, Hendrikse F, Carden D, Makridaki M, Murray IJ (2009) A new desktop instrument for measuring macular pigment optical density based on a novel technique for setting flicker thresholds. Ophthalmic Physiol Opt 29: 127-137

54. Hammond BR, Wooten BR, Smollon B (2005) Assessment of the validity of in vivo methods of measuring human macular pigment optical density. Optom Vis Sci 82: 387-404 55. Viner C (2003) Measuring macular pigment levels: an in-practice procedure? Optician 226: $24-26$

56. Delori FC, Goger DG, Hammond BR, Snodderly DM, Burns SA (2001) Macular pigment density measured by autofluorescence spectrometry: comparison with reflectometry and heterochromatic flicker photometry. J Opt Soc Am A Opt Image Sci Vis 18: 1212-1230 57. Neelam K, O'Gorman N, Nolan J, O'Donovan O, Wong HB, Eong KGA, Beatty S (2005) Measurement of macular pigment: Raman spectroscopy versus heterochromatic flicker photometry. Invest Ophthalmol Vis Sci 46: 1023-1032

58. van de Kraats J, Berendschot T, Valen S, van Norren D (2006) Fast assessment of the central macular pigment density with natural pupil using the macular pigment reflectometer. J Biomed Opt 11: 064031

59. Bone RA, Brener B, Gibert JC (2007) Macular pigment, photopigments, and melanin: distributions in young subjects determined by four-wavelength reflectometry. Vision Res 47: 3259-3268

60. Canovas R, Lima VC, Garcia P, Morini C, Prata TS, Rosen RB (2010) Comparison between macular pigment optical density measurements using two-wavelength autofluorescence and heterochromatic flicker photometry techniques. Invest Ophthalmol Vis Sci 51: 3152-3156

61. Werner JS, Wooten BR (1979) Opponent chromatic response functions for an average observer. Percept Psychophys 25: 371-374

62. Tang CY, Yip HS, Poon MY, Yau WL, Yap MKH (2004) Macular pigment optical density in young Chinese adults. Ophthalmic Physiol Opt 24: 586-593

63. Loane E, Stack J, Beatty S, Nolan JM (2007) Measurement of macular pigment optical density using two different heterochromatic flicker photometers. Curr Eye Res 32: 555-564 
64. Kirby ML, Galea M, Loane E, Stack J, Beatty S, Nolan JM (2009) Foveal anatomic associations with the secondary peak and the slope of the macular pigment spatial profile. Invest Ophthalmol Vis Sci 50: 1383-1391

65. Snodderly DM, Hammond BR (1999) In vivo psychophysical assessment of nutritional and environmental influences on human ocular tissues: lens and macular pigment. In: Taylor A (eds) Nutritional and Environmental Influences on the Eye. CRC Press, Boca Raton, FL, pp 251-273

66. Werner JS, Bieber ML, Schefrin BE (2000) Senescence of foveal and parafoveal cone sensitivities and their relations to macular pigment density. J Opt Soc Am A Opt Image Sci Vis 17: 1918-1932

67. Hammond BR, Johnson EJ, Russell RM, Krinsky NI, Yeum KJ, Edwards RB, Snodderly DM (1997) Dietary modification of human macular pigment density. Invest Ophthalmol Vis Sci 38: 1795-1801

68. Bone RA, Landrum JT, Gibert JC (2004) Macular pigment and the edge hypothesis of flicker photometry. Vision Res 44: 3045-3051

69. Burke JD, Curran-Celentano J, Wenzel AJ (2005) Diet and serum carotenoid concentrations affect macular pigment optical density in adults 45 years and older. $\mathrm{J}$ Nutr 135: $1208-1214$

70. Lam RF, Rao SK, Fan DSP, Lau FTC, Lam DSC (2005) Macular pigment optical density in a Chinese sample. Curr Eye Res 30: 799-805

71. Wooten BR, Hammond BR (2005) Spectral absorbance and spatial distribution of macular pigment using heterochromatic flicker photometry. Optom Vis Sci 82: 378-386 72. Stringham JM, Hammond BR (2007) The glare hypothesis of macular pigment function. Optom Vis Sci 84: 859-864

73. Wenzel AJ, Sheehan JP, Burke JD, Lefsrud MG, Curran-Celentano J (2007) Dietary intake and serum concentrations of lutein and zeaxanthin, but not macular pigment optical density, are related in spouses. Nutr Res 27: 462-469

74. Wenzel AJ, Sheehan JP, Gerweck C, Stringham JM, Fuld K, Curran-Celentano J (2007) Macular pigment optical density at four retinal loci during 120 days of lutein supplementation. Ophthalmic Physiol Opt 27: 329-335

75. Nolan JM, Stringham JM, Beatty S, Snodderly DM (2008) Spatial profile of macular pigment and its relationship to foveal architecture. Invest Ophthalmol Vis Sci 49: 2134-2142 76. Stringham JM, Hammond BR (2008) Macular pigment and visual performance under glare conditions. Optom Vis Sci 85: 82-88

77. Stringham JM, Hammond BR, Wooten BR, Snodderly DM (2006) Compensation for light loss resulting from filtering by macular pigment: relation to the S-cone pathway. Optom Vis Sci 83: 887-894

78. Connolly EE, Beatty S, Thurnham DI, Loughman J, Howard AN, Stack J, Nolan JM (2010) Augmentation of macular pigment following supplementation with all three macular carotenoids: an exploratory study. Curr Eye Res 35: 335-351

79. Loane E, McKay GJ, Nolan JM, Beatty S (2010) Apolipoprotein E genotype is associated with macular pigment optical density. Invest Ophthalmol Vis Sci 51: 2636-2643

80. Ciulla TA, Curran-Celantano J, Cooper DA, Hammond BR, Danis RP, Pratt LM, Riccardi KA, Filloon TG (2001) Macular pigment optical density in a midwestern sample.

Ophthalmology 108: 730-737

81. Ciulla TA, Hammond BR, Yung CW, Pratt LM (2001) Macular pigment optical density

before and after cataract extraction. Invest Ophthalmol Vis Sci 42: 1338-1341

82. Hammond BR, Fuld K, Curran-Celentano J (1995) Macular pigment density in monozygotic twins. Invest Ophthalmol Vis Sci 36: 2531-2541

83. Nolan JM, Stack J, Donovan OO, Loane E, Beatty S (2007) Risk factors for age-related maculopathy are associated with a relative lack of macular pigment. Exp Eye Res 84: 61-74 84. Nolan JM, Stack J, O'Connell E, Beatty S (2007) The relationships between macular pigment optical density and its constituent carotenoids in diet and serum. Invest Ophthalmol Vis Sci 48: 571-582 
85. Schalch W, Cohn W, Barker FM, Kopcke W, Mellerio J, Bird AC, Robson AG, Fitzke FF, van Kuijk FJGM (2007) Xanthophyll accumulation in the human retina during supplementation with lutein or zeaxanthin - the LUXEA (LUtein Xanthophyll Eye

Accumulation) study. Arch Biochem Biophys 458: 128-135

86. Hammond BR, Wooten BR (2005) CFF thresholds: relation to macular pigment optical density. Ophthalmic Physiol Opt 25: 315-319

87. Cooper DA, Curran-Celentano J, Ciulla TA, Hammond BR, Danis RB, Pratt LM, Riccardi $\mathrm{KA}$, Filloon TG (2000) Olestra consumption is not associated with macular pigment optical density in a cross-sectional volunteer sample in Indianapolis. J Nutr 130: 642-647

88. Curran-Celentano J, Hammond BR, Ciulla TA, Cooper DA, Pratt LM, Danis RB (2001)

Relation between dietary intake, serum concentrations, and retinal concentrations of lutein and zeaxanthin in adults in a Midwest population. Am J Clin Nutr 74: 796-802

89. Bone RA, Landrum JT, Guerra LH, Ruiz CA (2003) Lutein and zeaxanthin dietary supplements raise macular pigment density and serum concentrations of these carotenoids in humans. J Nutr 992-998

90. Bone RA, Landrum JT, Cao Y, Howard AN, Alvarez-Calderon F (2007) Macular pigment response to a supplement containing meso-zeaxanthin, lutein and zeaxanthin. Nutr Metab 4: 12

91. Koh HH, Murray IJ, Nolan D, Carden D, Feather J, Beatty S (2004) Plasma and macular response to lutein supplement in subjects with and without age-related maculopathy: a pilot study. Exp Eye Res 79: 21-27

92. Robson AG, Holder GE, Moreland JD, Kulikowski JJ (2006) Chromatic VEP assessment of human macular pigment: comparison with minimum motion and minimum flicker profiles. Vis Neurosci 23: 275-283

93. Engles M, Wooten B, Hammond B (2007) Macular pigment: a test of the acuity hypothesis. Invest Ophthalmol Vis Sci 48: 2922-2931

94. Rodriguez-Carmona M, Kvansakul J, Harlow JA, Kopcke W, Schalch W, Barbur JL (2006) The effects of supplementation with lutein and/or zeaxanthin on human macular pigment density and colour vision. Ophthalmic Physiol Opt 26: 137-147

95. Kvansakul J, Rodriguez-Carmona M, Edgar DF, Barker FM, Kopcke W, Schalch W, Barbur JL (2006) Supplementation with the carotenoids lutein or zeaxanthin improves human visual performance. Ophthalmic Physiol Opt 26: 362-371

96. Makridaki M, Carden D, Murray IJ (2009) Macular pigment measurement in clinics: controlling the effect of the ageing media. Ophthalmic Physiol Opt 29: 338-344

97. Nolan JM, Kenny R, O'Regan C, Cronin H, Loughman J, Connolly EE, Kearney P, Loane E, Beatty S (2010) Macular Pigment Optical Density in an Ageing Irish Population: The Irish Longitudinal Study on Ageing. Ophthalmic Res 44: 131-139

98. Wüstemeyer H, Jahn C, Nestler A, Barth T, Wolf S (2002) A new instrument for the quantification of macular pigment density: first results in patients with AMD and healthy subjects. Graefes Arch Clin Exp Ophthalmol 240: 666-671

99. Curcio CA, Allen KA, Sloan KR, Lerea CL, Hurley JB, Klock IB, Milam AH (1991)

Distribution and morphology of human cone photoreceptors stained with anti-blue opsin. $\mathrm{J}$

Comp Neurol 312: 610-624

100. Curcio CA, Sloan KR (1992) Packing geometry of human cone photoreceptors variation with eccentricity and evidence for local anisotropy. Vis Neurosci 9: 169-180 101. Curcio CA, Sloan KR, Kalina RE, Hendrickson AE (1990) Human photoreceptor topography. J Comp Neurol 292: 497-523

102. Cicerone CM, Nerger JL (1989) The relative numbers of long-wavelength-sensitive to middle-wavelength-sensitive cones in the human fovea centralis. Vision Res 29: 115-128 103. Nerger JL, Cicerone CM (1992) The ratio of L-cones to M-cones in the human parafoveal retina. Vision Res 32: 879-888

104. Knau H, Jagle H, Sharpe LT (2001) L/M cone ratios as a function of retinal eccentricity. Color Res Appl 26: S128-S132 
105. Hammond BR, Wooten BR, Snodderly DM (1998) Preservation of visual sensitivity of older subjects: association with macular pigment density. Invest Ophthalmol Vis Sci 39: 397406

106. Brindley GS, Du Croz JJ, Rushton WA (1966) The flicker fusion frequency of the bluesensitive mechanism of colour vision. J Physiol 183: 497-500

107. Nolan J, O'Donovan O, Kavanagh H, Stack J, Harrison M, Muldoon A, Mellerio J, Beatty S (2004) Macular pigment and percentage of body fat. Invest Ophthalmol Vis Sci 45: 3940-3950

108. Chen SF, Chang Y, Wu JC (2001) The spatial distribution of macular pigment in humans. Curr Eye Res 23: 422-434

109. Berendschot T, van Norren D (2004) Objective determination of the macular pigment optical density using fundus reflectance spectroscopy. Arch Biochem Biophys 430: 149-155 110. Bernstein PS, Zhao DY, Sharifzadeh M, Ermakov IV, Gellermann W (2004) Resonance Raman measurement of macular carotenoids in the living human eye. Arch Biochem Biophys 430: 163-169

111. Gellermann W, Bernstein PS (2004) Noninvasive detection of macular pigments in the human eye. J Biomed Opt 9: 75-85

112. Bhosale P, Zhao DY, Bernstein PS (2007) HPLC measurement of ocular carotenoid levels in human donor eyes in the lutein supplementation era. Invest Ophthalmol Vis Sci 48: 543-549

113. Hammond BR, Ciulla TA, Snodderly DM (2002) Macular pigment density is reduced in obese subjects. Invest Ophthalmol Vis Sci 43: 47-50

114. Gallaher KT, Mura M, Todd WA, Harris TL, Kenyon E, Harris T, Johnson KC, Satterfield S, Kritchevsky SB, lannaccone A, HIth ABCS (2007) Estimation of macular pigment optical density in the elderly: test-retest variability and effect of optical blur in pseudophakic subjects. Vision Res 47: 1253-1259

115. Snodderly DM, Handelman GJ, Adler AJ (1991) Distribution of individual macular pigment carotenoids in central retina of macaque and squirrel-monkeys. Invest Ophthalmol Vis Sci 32: 268-279

116. Berendschot T, van Norren D (2006) Macular pigment shows ringlike structures. Invest Ophthalmol Vis Sci 47: 709-714

117. Delori FC, Goger DG, Keilhauer C, Salvetti P, Staurenghi G (2006) Bimodal spatial distribution of macular pigment: evidence of a gender relationship. J Opt Soc Am A Opt Image Sci Vis 23: 521-538

118. Wyszecki G, Stiles WS (1982) The Eye. In: (eds) Color Science: Concepts and Methods, Quantitative Data and Formulae. Wiley-Interscience, New York, pp 112-114 119. Bartlett H, Acton J, Eperjesi F (2010) Clinical evaluation of the MacuScope macular pigment densitometer. Br J Ophthalmol 94: 328-331

120. Hagen S, Krebs I, Glittenberg C, Binder S (2010) Repeated measures of macular pigment optical density to test reproducibility of heterochromatic flicker photometry. Acta Ophthalmol 88: 207-211

121. van der Veen RLP, Berendschot T, Makridaki M, Hendrikse F, Carden D, Murray IJ (2009) Correspondence between retinal reflectometry and a flicker-based technique in the measurement of macular pigment spatial profiles. J Biomed Opt 14: 064046

122. Johnson EJ, Chung HY, Caldarella SM, Snodderly DM (2008) The influence of supplemental lutein and docosahexaenoic acid on serum, lipoproteins, and macular pigmentation. Am J Clin Nutr 87: 1521-1529

123. Troxler DIPV (1804) Über das Verschwinden gegebener Gegenstände innerhalb unseres Gesichtskreises. In: Himly J, Schmidt JA (eds) Ophthalmologische Bibliothek. Jena: Fromann, pp 1-53

124. Stumpf $P$ (1911) On the dependence of the visual sensation of movement and its negative aftereffect on the stimulation processes on the retina. Z Psychol 59: 321-330 125. Todorović D (1996) A gem from the past: Pleikart Stumpf's (1911) anticipation of the aperture problem, Reichardt detectors, and perceived motion loss at equiluminance.

Perception 25: 1235-1242 
126. Moreland JD (1980) A modified Moreland anomaloscope using optokinetic nystagmus to define colour matches objectively. In: Verriest G (eds) Colour Vision Deficiencies V.

Hilger, Bristol, pp 189-191

127. Moreland JD (1982) Spectral sensitivity measurements by motion photometry. Doc Ophthalmol Proc Ser 33: 61-66

128. Anstis SM, Cavanagh $P$ (1983) A minimum motion technique for judging equiluminance. In: Mollon JD, Sharpe LT (eds) Colour Vision: Physiology and Psychophysics. Academic Press, London, pp 155-166

129. Robson AG, Harding G, van Kuijk F, Pauleikhoff D, Holder GE, Bird AC, Fitzke FW, Moreland JD (2005) Comparison of fundus autofluorescence and minimum-motion

measurements of macular pigment distribution profiles derived from identical retinal areas.

Perception 34: 1029-1034

130. West P, Mellerio J (2005) Measuring MPOD using a CRT display [online].

http://www.crsltd.com/research-topics/macular-pigment/index.html and

http://www.crsltd.com/catalog/metropsis/MP.html. Accessed 1 May 2009

131. Brindley GS, Willmer EN (1952) The reflexion of light from the macular and peripheral fundus oculi in man. J Physiol 116: 350-356

132. Berendschot T, DeLint PJ, van Norren D (2003) Fundus reflectance - historical and present ideas. Prog Retin Eye Res 22: 171-200

133. van Norren D, Tiemeijer LF (1986) Spectral reflectance of the human eye. Vision Res 26: 313-320

134. Delori FC, Pflibsen KP (1989) Spectral reflectance of the human ocular fundus. Appl Opt 28: 1061-1077

135. Elsner AE, Burns SA, Beausencourt E, Weiter JJ (1998) Foveal cone photopigment distribution: small alterations associated with macular pigment distribution. Invest Ophthalmol Vis Sci 39: 2394-2404

136. Berendschot T, Goldbohm RA, Klopping WAA, van de Kraats J, van Norel J, van Norren D (2000) Influence of lutein supplementation on macular pigment, assessed with two objective techniques. Invest Ophthalmol Vis Sci 41: 3322-3326

137. Bour LJ, Koo L, Delori FC, Apkarian P, Fulton AB (2002) Fundus photography for measurement of macular pigment density distribution in children. Invest Ophthalmol Vis Sci 43: $1450-1455$

138. Cardinault N, Gorrand JM, Tyssandier V, Grolier P, Rock E, Borel P (2003) Short-term supplementation with lutein affects biomarkers of lutein status similarly in young and elderly subjects. Exp Gerontol 38: 573-582

139. van de Kraats J, van Norren D (2008) Directional and nondirectional spectral reflection from the human fovea. J Biomed Opt 13: 024010

140. van de Kraats J, Berendschot T, van Norren D (1996) The pathways of light measured in fundus reflectometry. Vision Res 36: 2229-2247

141. Berendschot T, van Norren D (2005) On the age dependency of the macular pigment optical density. Exp Eye Res 81: 602-609

142. Kanis MJ, Berendschot T, van Norren D (2007) Influence of macular pigment and melanin on incident early AMD in a white population. Graefes Arch Clin Exp Ophthalmol 245: 767-773

143. Kanis MJ, Berendschot T, van Norren D (2007) Interocular agreement in melanin and macular pigment optical density. Exp Eye Res 84: 934-938

144. Berendschot T, Willemse-Assink JJM, Bastiaanse M, de Jong P, van Norren D (2002) Macular pigment and melanin in age-related maculopathy in a general population. Invest Ophthalmol Vis Sci 43: 1928-1932

145. Chang Y, Lee FL, Chen SJ, Chen SF (2002) Optical measurement of human retinal macular pigment and its spatial distribution with age. Med Phys 29: 2621-2628

146. Zagers NPA, van de Kraats J, Berendschot T, van Norren D (2002) Simultaneous measurement of foveal spectral reflectance and cone-photoreceptor directionality. Appl Opt 41: 4686-4696 
147. Zagers NPA, van Norren D (2004) Absorption of the eye lens and macular pigment derived from the reflectance of cone photoreceptors. J Opt Soc Am A Opt Image Sci Vis 21: 2257-2268

148. Kanis MJ, Wisse RPL, Berendschot T, van de Kraats J, van Norren D (2008) Foveal cone-photoreceptor integrity in aging macula disorder. Invest Ophthalmol Vis Sci 49: 20772081

149. Elsner AE, Burns SA, Delori FC, Webb RH (1990) Quantitative reflectometry with the SLO. In: Naseman JE, Burk ROW (eds) Scanning Laser Ophthalmoscopy and Tomography. Quintessenz-Verlag, Munich, pp 109-121

150. Seth R, Gouras P (2004) Assessing macular pigment from SLO images. Doc Ophthalmol 108: 197-202

151. Helb HM, Issa PC, Van der Veen RLP, Berendschot T, Scholl HPN, Holz FG (2008)

Abnormal macular pigment distribution in type 2 idiopathic macular telangiectasia. Retina 28: 808-816

152. van de Kraats J, Kanis MJ, Genders SW, van Norren D (2008) Lutein and zeaxanthin measured separately in the living human retina with fundus reflectometry. Invest Ophthalmol Vis Sci 49: 5568-5573

153. Sharifzadeh M, Bernstein PS, Gellermann W (2006) Nonmydriatic fluorescence-based quantitative imaging of human macular pigment distributions. J Opt Soc Am A Opt Image Sci Vis 23: $2373-2387$

154. Wolf-Schnurrbusch UEK, Roosli N, Weyermann E, Heldner MR, Hohne K, Wolf S (2007) Ethnic differences in macular pigment density and distribution. Invest Ophthalmol Vis Sci 48: 3783-3787

155. Bernstein PS, Delori FC, Richer S, van Kuijk FJM, Wenzel AJ (2010) The value of measurement of macular carotenoid pigment optical densities and distributions in agerelated macular degeneration and other retinal disorders. Vision Res 50: 716-728

156. Delori FC (1994) Spectrophotometer for noninvasive measurement of intrinsic fluorescence and reflectance of the ocular fundus. Appl Opt 33: 7439-7452

157. Delori FC, Dorey CK, Staurenghi G, Arend O, Goger DG, Weiter JJ (1995) In vivo fluorescence of the ocular fundus exhibits retinal pigment epithelium lipofuscin characteristics. Invest Ophthalmol Vis Sci 36: 718-729

158. von Ruckmann A, Fitzke FW, Bird AC (1995) Distribution of fundus autofluorescence with a scanning laser ophthalmoscope. Br J Ophthalmol 79: 407-412

159. Delori FC (2004) Autofluorescence method to measure macular pigment optical densities fluorometry and autofluorescence imaging. Arch Biochem Biophys 430: 156-162 160. Schmitz-Valckenberg S, Holz FG, Bird AC, Spaide RF (2008) Fundus autofluorescence imaging - review and perspectives. Retina 28: 385-409

161. Liew SHM, Gilbert CE, Spector TD, Mellerio J, Van Kuijk FJ, Beatty S, Fitzke F, Marshall J, Hammond CJ (2006) Central retinal thickness is positively correlated with macular pigment optical density. Exp Eye Res 82: 915-920

162. Trieschmann M, Heimes B, Hense HW, Pauleikhoff D (2006) Macular pigment optical density measurement in autofluorescence imaging: comparison of one- and two-wavelength methods. Graefes Arch Clin Exp Ophthalmol 244: 1565-1574

163. Jahn C, Wustemeyer H, Brinkmann C, Trautmann S, Mossner A, Wolf S (2005) Macular pigment density in age-related maculopathy. Graefes Arch Clin Exp Ophthalmol 243: $222-227$

164. Liew SHM, Gilbert CE, Spector TD, Mellerio J, Marshall J, van Kuijk FJ, Beatty S, Fitzke F, Hammond CJ (2005) Heritability of macular pigment: a twin study. Invest Ophthalmol Vis Sci 46: 4430-4436

165. Delori FC, Goger DG, Dorey CK (2001) Age-related accumulation and spatial distribution of lipofuscin in RPE of normal subjects. Invest Ophthalmol Vis Sci 42: 1855-1866 166. Bernstein PS, Yoshida MD, Katz NB, McClane RW, Gellermann W (1998) Raman detection of macular carotenoid pigments in intact human retina. Invest Ophthalmol Vis Sci 39: 2003-2011 
167. Koyama Y, Takatsuka I, Nakata M, Tasumi M (1988) Raman and infrared spectra of the all-trans, 7-cis, 9-cis, 13-cis and 15-cis isomers of beta carotene - key bands distinguishing stretched or terminal-bent configurations from central-bent configurations. J Raman Spectrosc 19: 37-49

168. Ermakov IV, McClane RW, Gellermann W, Bernstein PS (2001) Resonant Raman detection of macular pigment levels in the living human retina. Opt Lett 26: 202-204 169. Bernstein PS (2002) New insights into the role of the macular carotenoids in agerelated macular degeneration. Resonance Raman studies. Pure Appl Chem 74: 1419-1425 170. Bernstein PS, Zhao DY, Wintch SW, Ermakov IV, McClane RW, Gellermann W (2002) Resonance Raman measurement of macular carotenoids in normal subjects and in agerelated macular degeneration patients. Ophthalmology 109: 1780-1787

171. Gellermann W, Ermakov IV, Ermakova MR, McClane RW, Zhao DY, Bernstein PS (2002) In vivo resonant Raman measurement of macular carotenoid pigments in the young and the aging human retina. J Opt Soc Am A Opt Image Sci Vis 19: 1172-1186

172. Gellermann W, Ermakov IV, McClane RW, Bernstein PS (2002) Raman imaging of human macular pigments. Opt Lett 27: 833-835

173. Zhao DY, Wintch SW, Ermakov IV, Gellermann W, Bernstein PS (2003) Resonance Raman measurement of macular carotenoids in retinal, choroidal, and macular dystrophies. Arch Ophthalmol 121: 967-972

174. Ermakov I, Ermakova M, Gellermann W, Bernstein PS (2004) Macular pigment Raman detector for clinical applications. J Biomed Opt 9: 139-148

175. Ermakov IV, Sharifzadeh M, Ermakova M, Gellermann W (2005) Resonance Raman detection of carotenoid antioxidants in living human tissue. J Biomed Opt 10: 064028 176. Hogg RE, Anderson RS, Stevenson MR, Zlatkova MB, Chakravarthy U (2007) In vivo macular pigment measurements: a comparison of resonance Raman spectroscopy and heterochromatic flicker photometry. Br J Ophthalmol 91: 485-490

177. Obana A, Hiramitsu T, Gohto Y, Ohira A, Mizuno S, Hirano T, Bernstein PS, Fujii H, Iseki K, Tanito M, Hotta Y (2008) Macular carotenoid levels of normal subjects and agerelated maculopathy patients in a Japanese population. Ophthalmology 115: 147-157 178. Sharifzadeh M, Zhao DY, Bernstein PS, Gellermann W (2008) Resonance Raman imaging of macular pigment distributions in the human retina. J Opt Soc Am A Opt Image Sci Vis 25: 947-957

179. Hogg RE, Zlatkova MB, Chakravarthy U, Anderson RS (2007) Investigation of the effect of simulated lens yellowing, transparency loss and refractive error on in vivo resonance Raman spectroscopy. Ophthalmic Physiol Opt 27: 225-231

180. Bernstein PS, Gellermann W (2003) Author response: assessment of the Raman method of measuring human macular pigment [Letter] [online]. Investigative Ophthalmology \& Visual Science. Available at:

http://www.iovs.org/cgi/eletters?lookup=by date\&days=9999\#74. Accessed 15 September 2009

181. Bernstein PS, Gellermann W (2003) Author response: assessment of the Raman method of measuring human macular pigment (II) [Letter] [online]. Investigative

Ophthalmology \& Visual Science. Available at:

http://www.iovs.org/cgi/eletters?lookup=by date\&days=9999\#74. Accessed 15 September 2009

182. Wooten BR, Hammond BR (2003) Assessment of the Raman method of measuring human macular pigment [Letter] [online]. Investigative Ophthalmology \& Visual Science. Available at: http://www.iovs.org/cgi/eletters?lookup=by date\&days=9999\#73. Accessed 15 September 2009

183. Wooten BR, Hammond BR (2003) Assessment of the Raman method of measuring human macular pigment (II) [Letter] [online]. Investigative Ophthalmology \& Visual Science. Available at: http://www.iovs.org/cgi/eletters?lookup=by date\&days=9999\#73. Accessed 15 September 2009

184. Hammond BR, Wooten BR (2005) Resonance Raman spectroscopic measurement of carotenoids in the skin and retina. J Biomed Opt 10: 054002 
185. Gellermann W, Bernstein PS (2006) Assessment of the validity of in vivo methods of measuring human macular pigment optical density [Letter]. Optom Vis Sci 83: 254-255

186. Hammond BR, Wooten BR, Smollon B (2006) Assessment of the validity of in vivo methods of measuring human macular pigment optical density - Authors' response [Letter]. Optom Vis Sci 83: 256-259

187. Beatty S, van Kuijk F, Chakravarthy U (2008) Macular pigment and age-related macular degeneration: longitudinal data and better techniques of measurement are needed. Invest Ophthalmol Vis Sci 49: 843-845

188. Bird AEC, Bressler NM, Bressler SB, Chisholm IH, Coscas G, Davis MD, Dejong P, Klaver CCW, Klein BEK, Klein R, Mitchell P, Sarks JP, Sarks SH, Sourbane G, Taylor HR, Vingerling JR (1995) An international classification and grading system for age-related maculopathy and age-related macular degeneration. Surv Ophthalmol 39: 367-374 
Figure 1

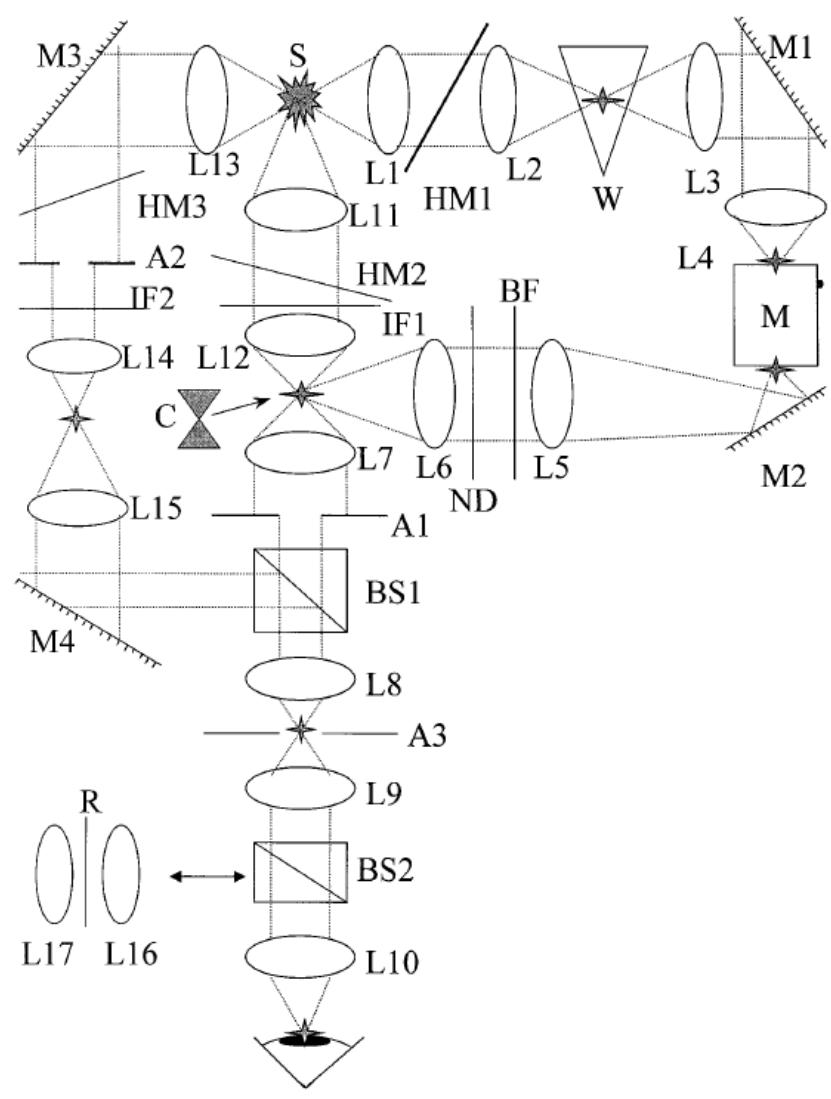


Figure 2

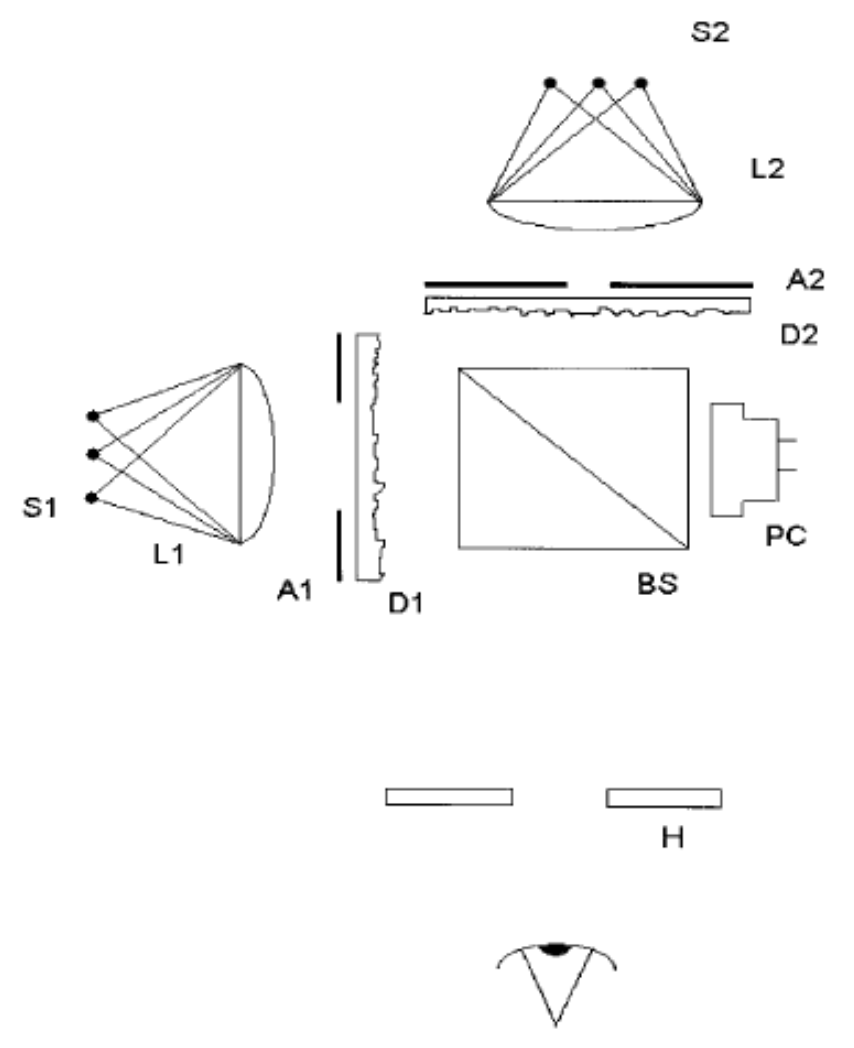


Figure 4

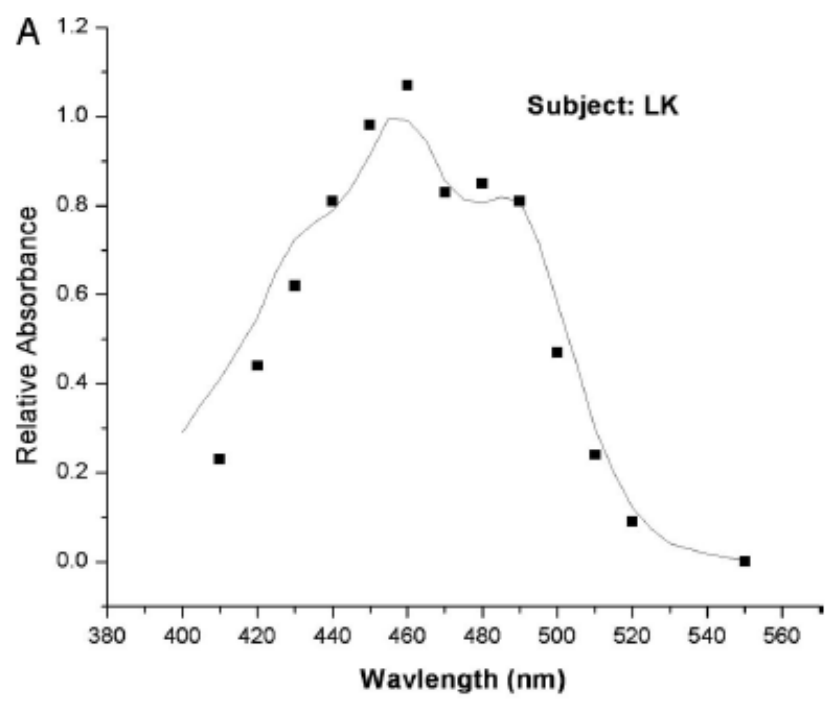


Figure 6
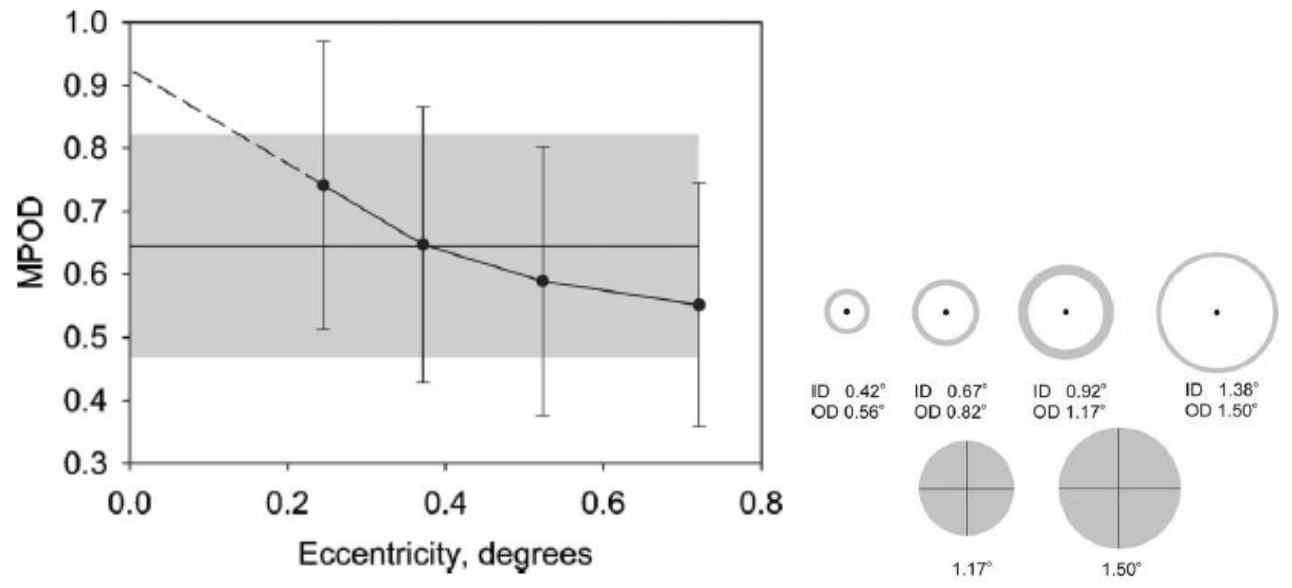
Figure 7

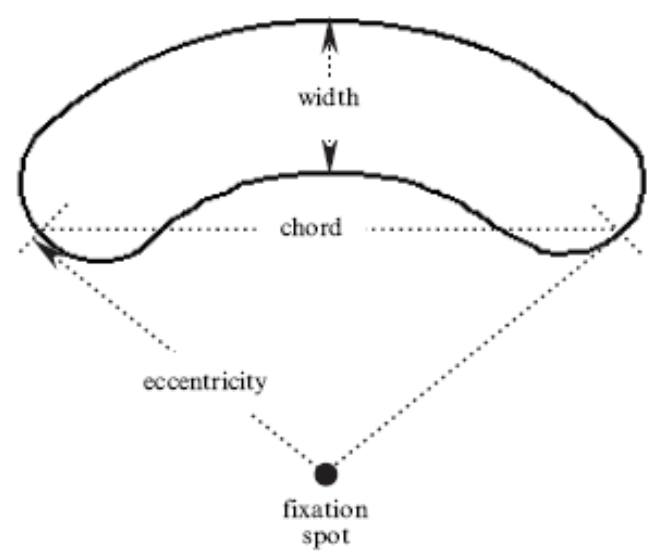

Foveal field/deg

0.9

eocentricity $/{ }^{\circ}$ chord $/$ deg width $/$ deg

\begin{tabular}{lll}
\hline 0.8 & 1.8 & \\
1.2 & 2.1 & 0.8 \\
1.7 & 2.8 & \\
2.3 & 3.8 & \\
2.8 & 4.7 & \\
3.3 & 5.2 & \\
3.9 & 6.1 & 1.1 \\
4.4 & 6.0 & \\
5.3 & 6.0 & \\
6.6 & 5.9 & \\
7.5 & 6.0 & \\
\hline
\end{tabular}

ixation

spot
Diameters/deg

Annuli 2.2 
Figure 9

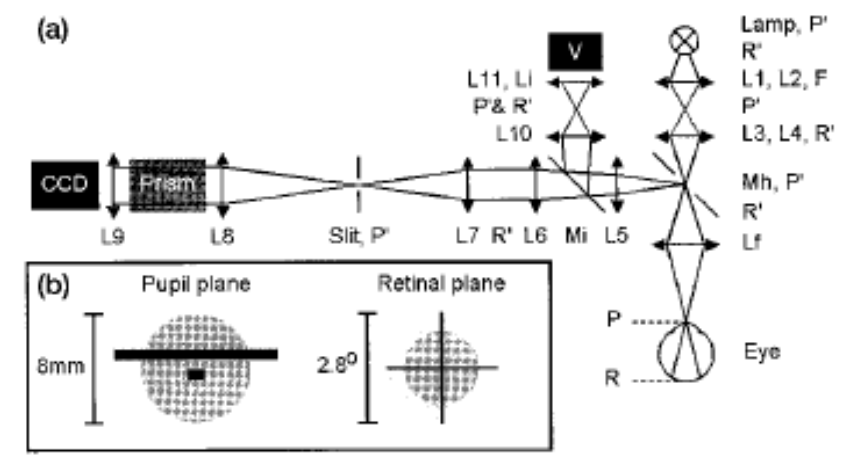


Figure 10

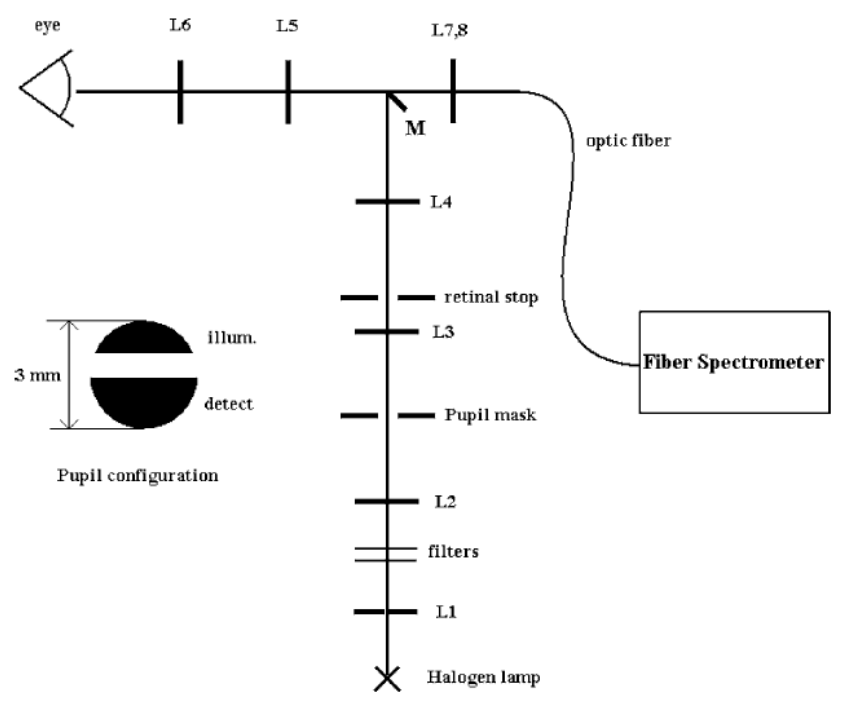



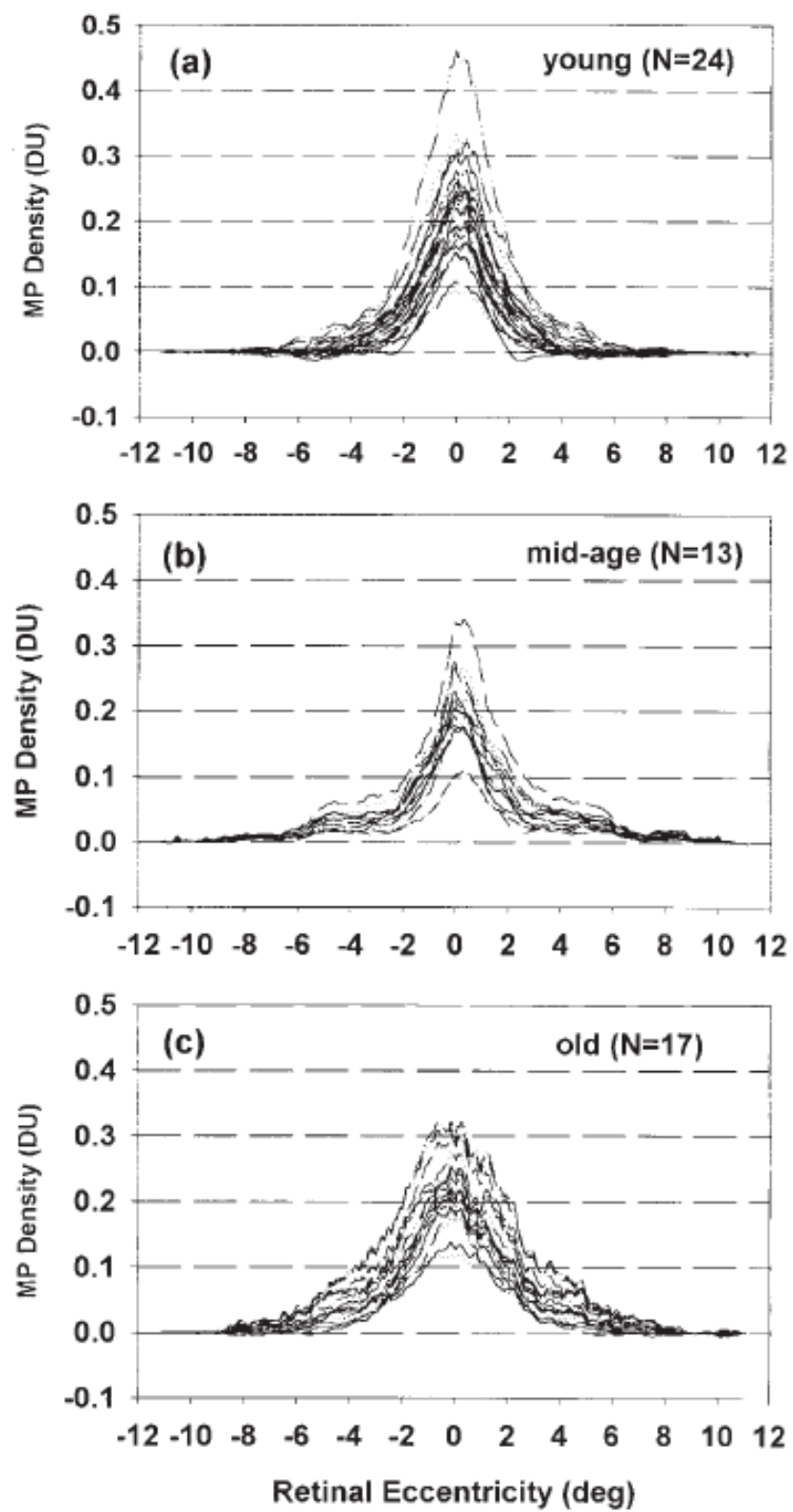
A

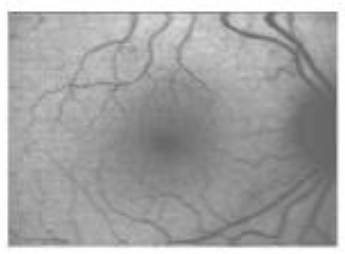

B

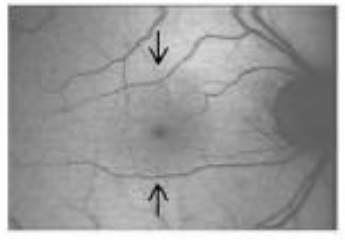

C

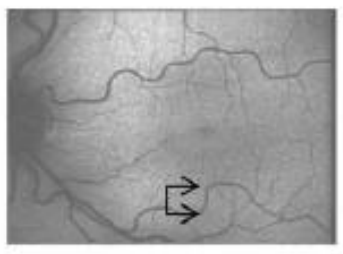

D

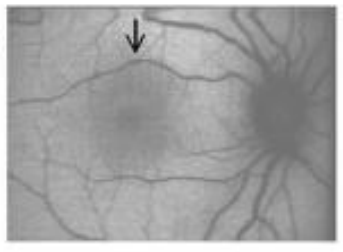

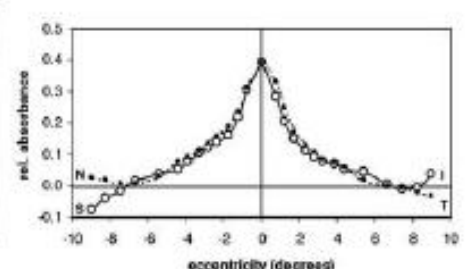

ascontricky [dogrova)
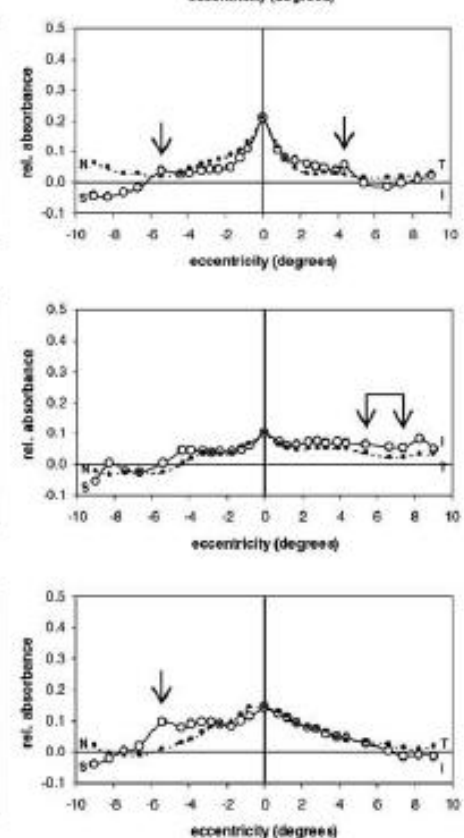

E
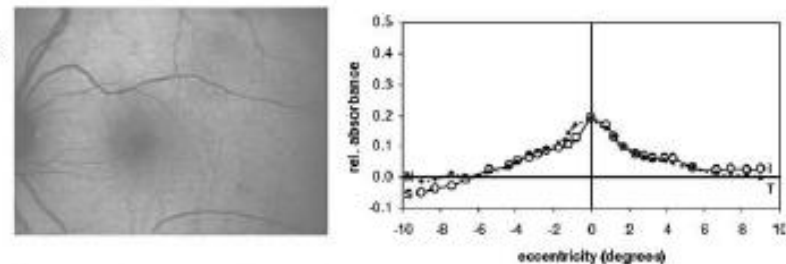

F
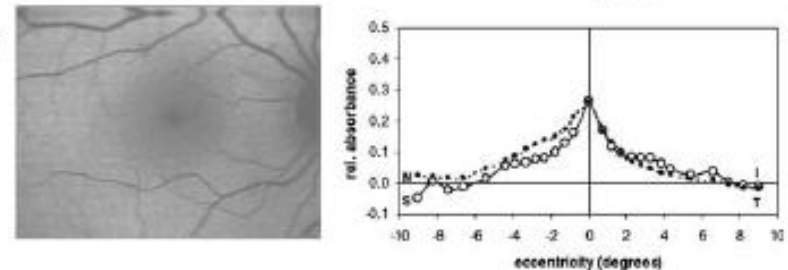

G
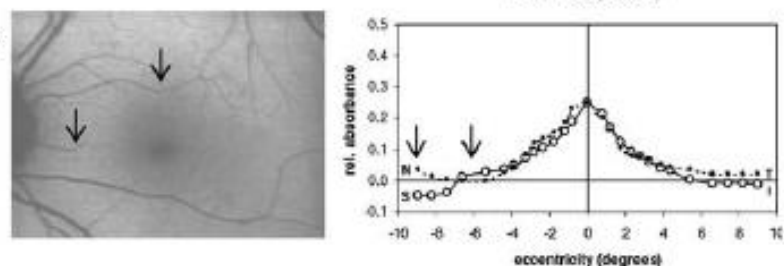

H
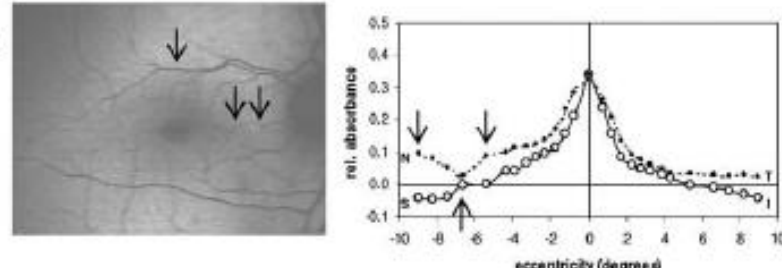


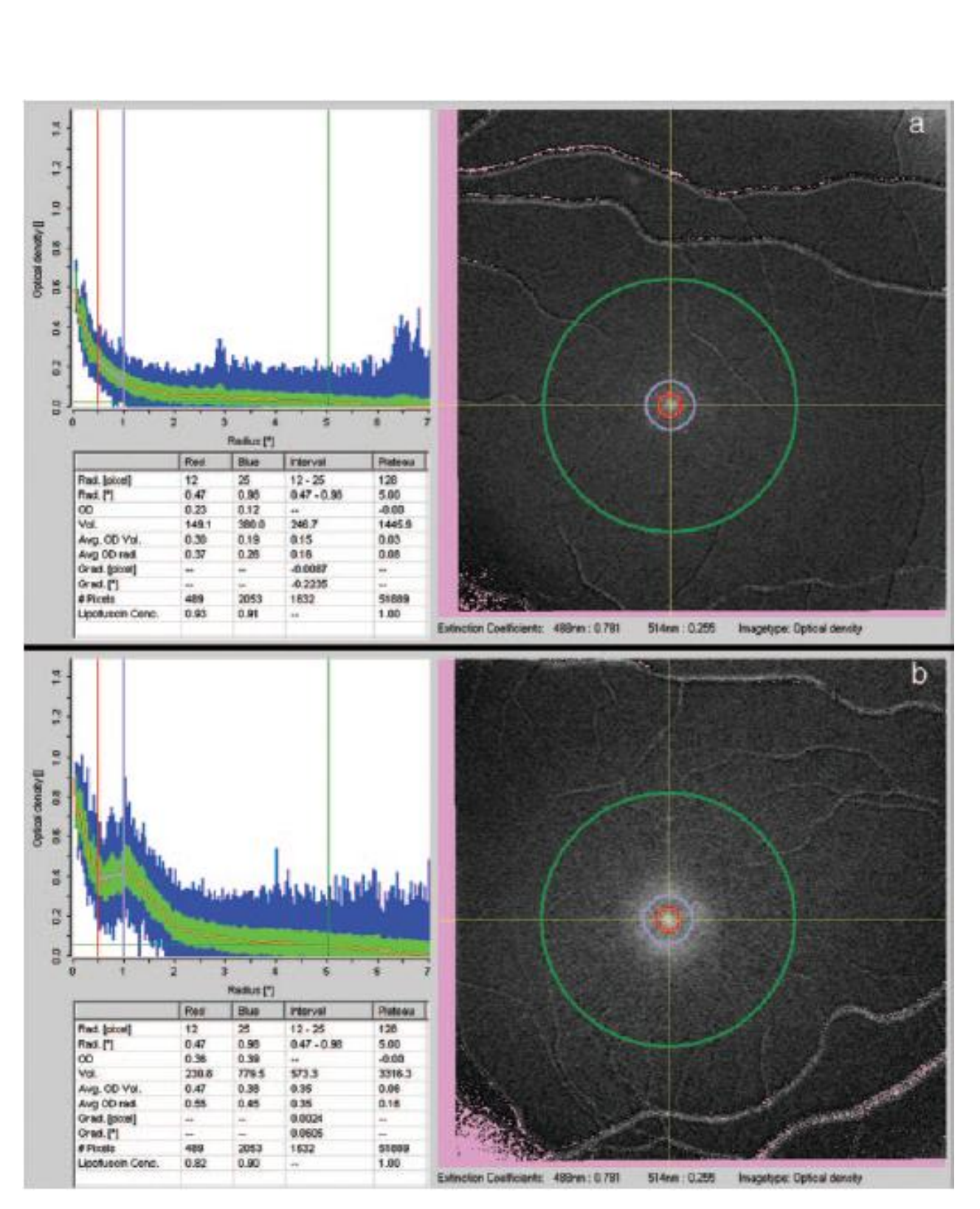

Figure 14
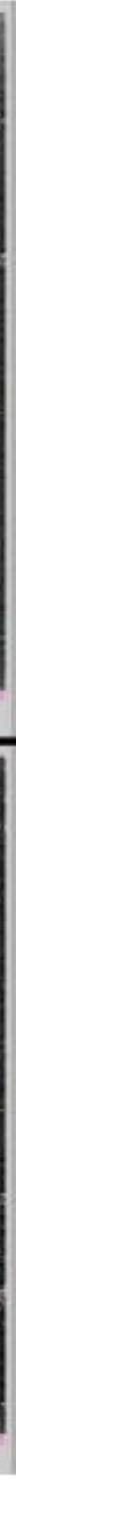

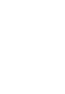
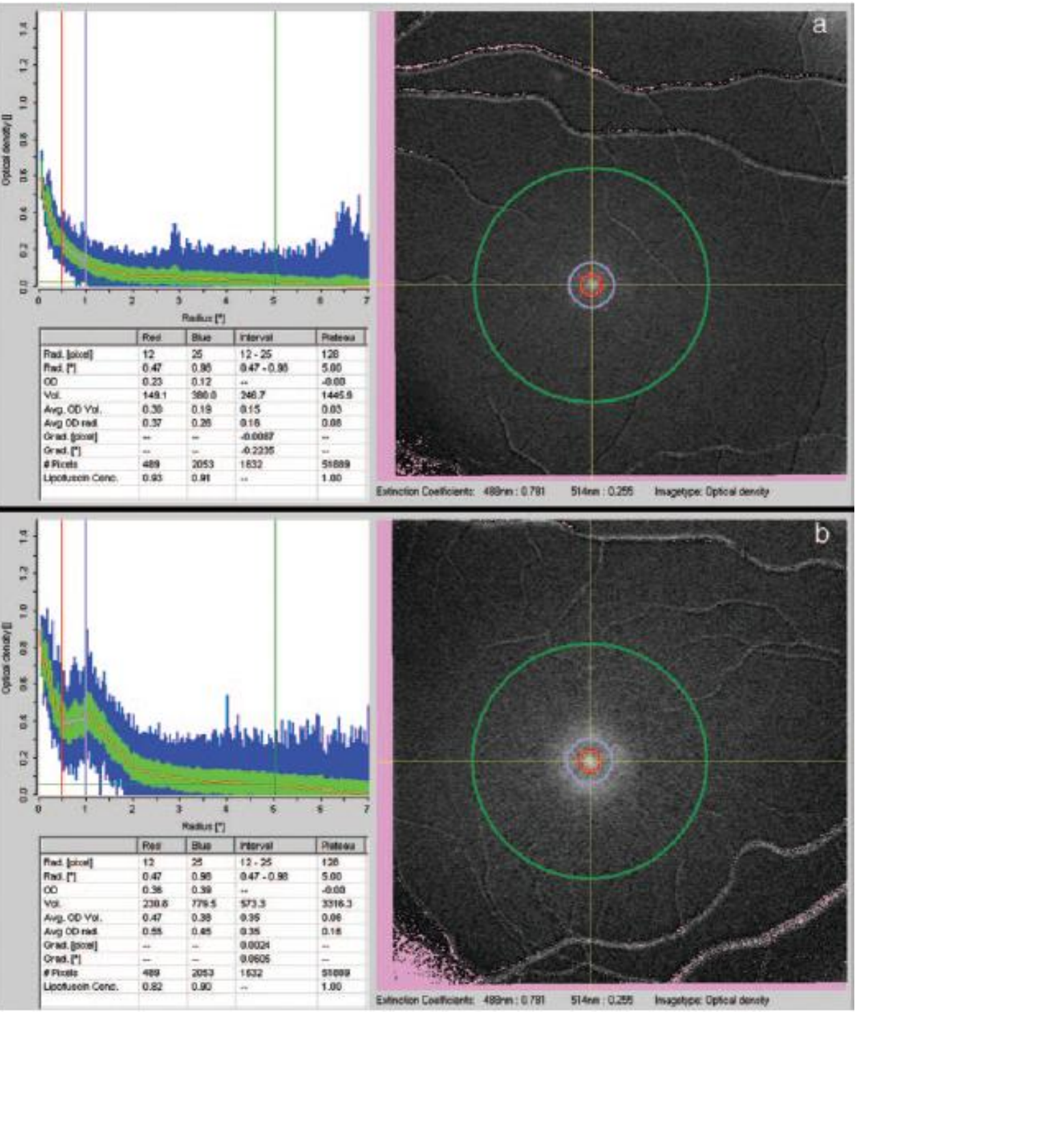
Figure 15

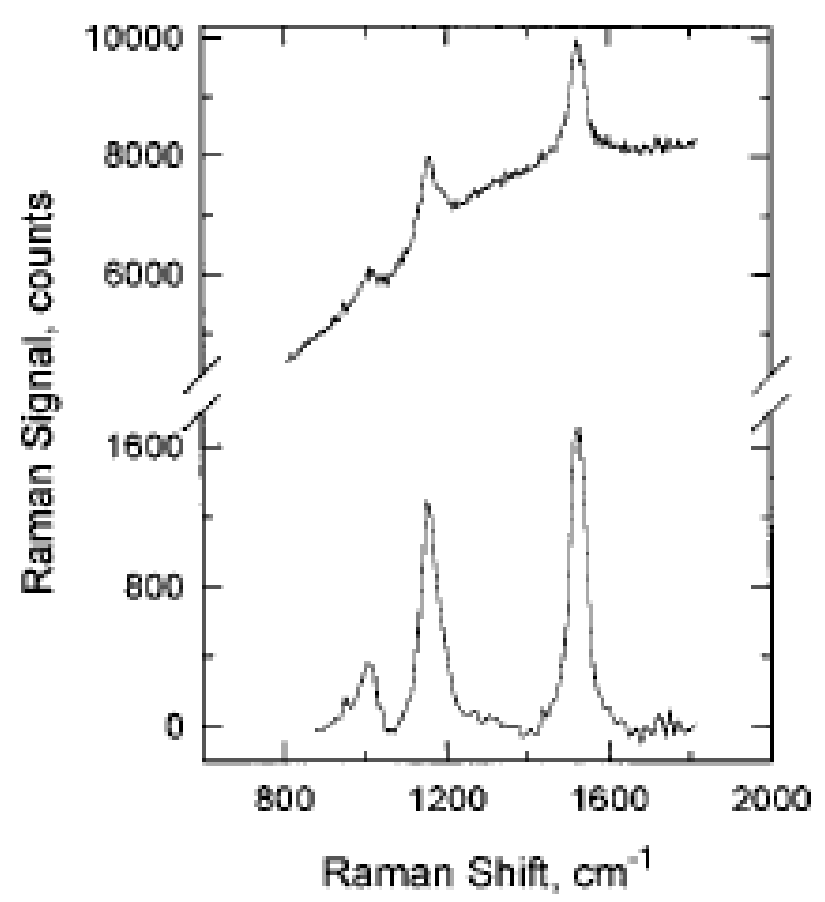


Figure 16

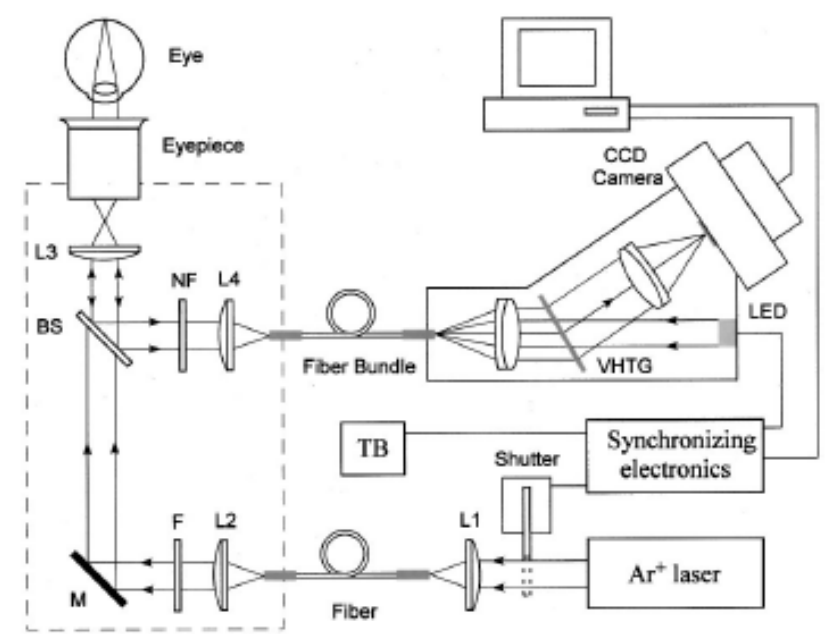


Figure 17

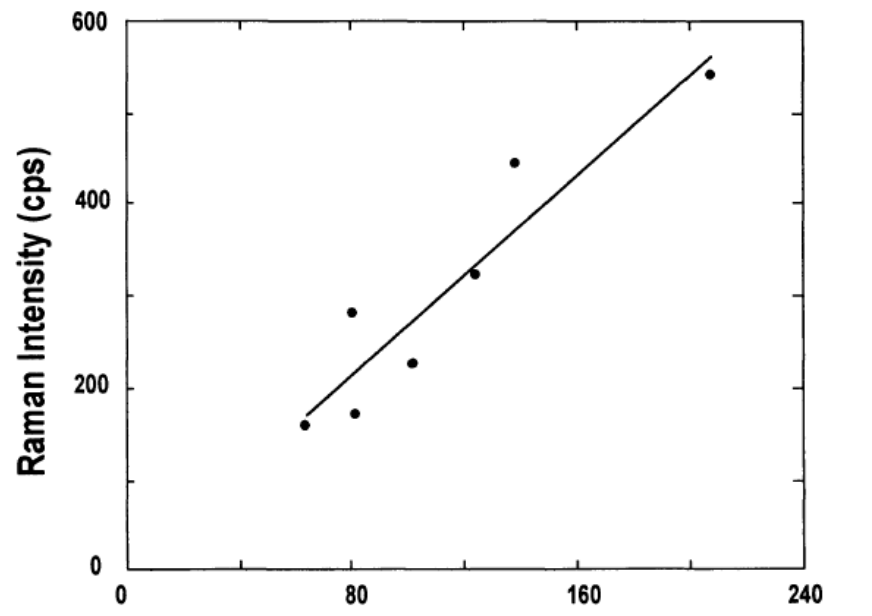

Carotenoid Content in $5 \mathrm{~mm}$ Macular Sample / $\mathrm{ng}$

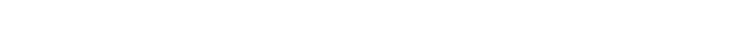

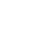




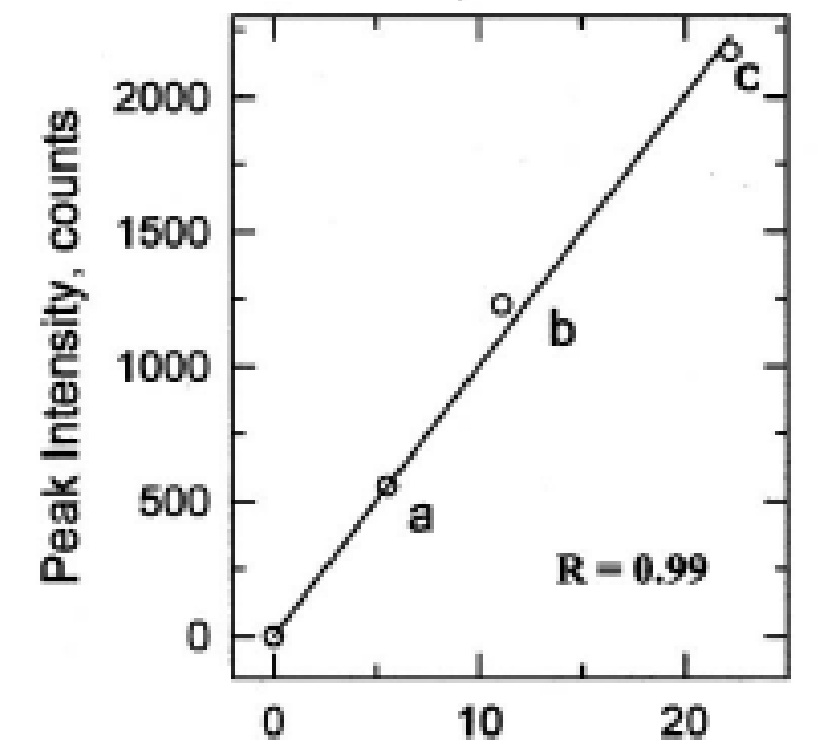

Concentration, ng/smpl.area

Figure 18 

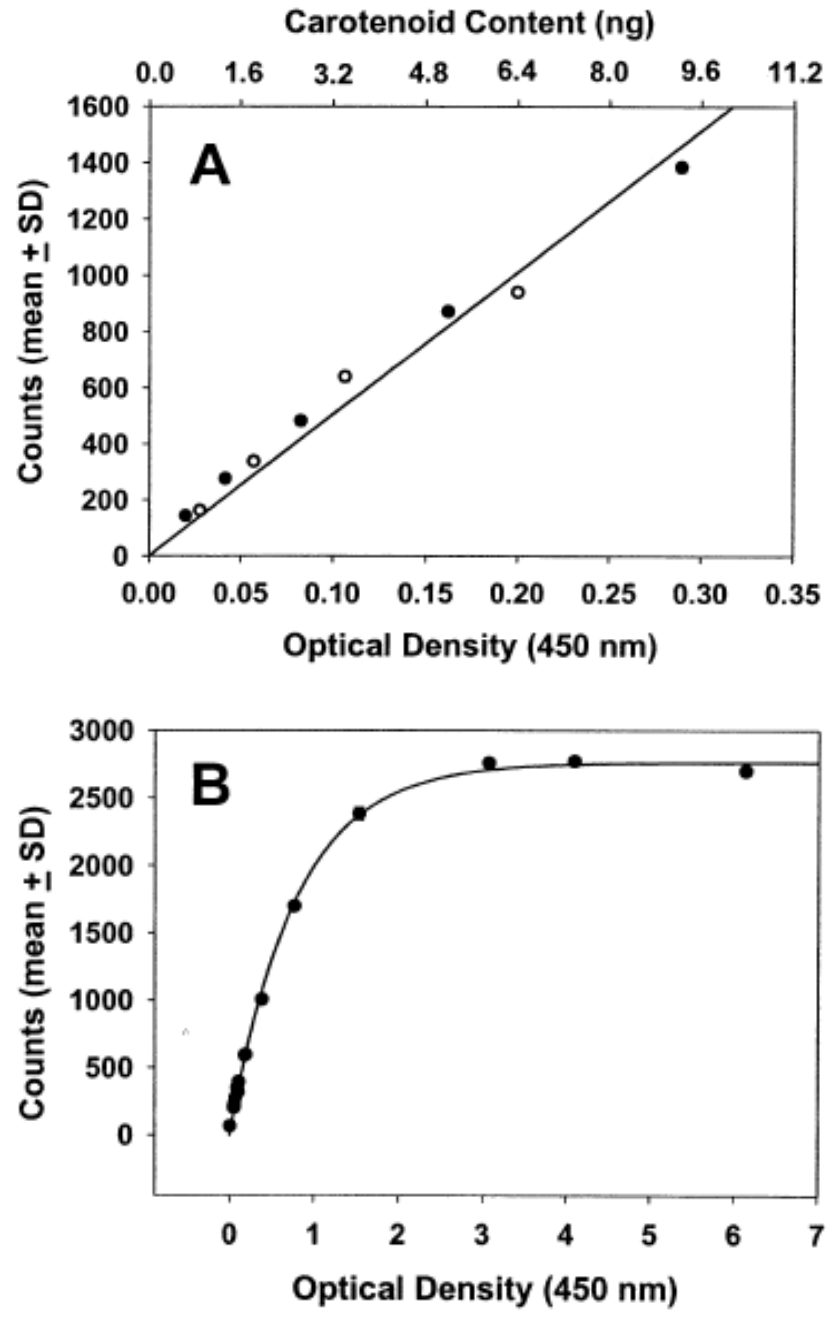
Figure 21

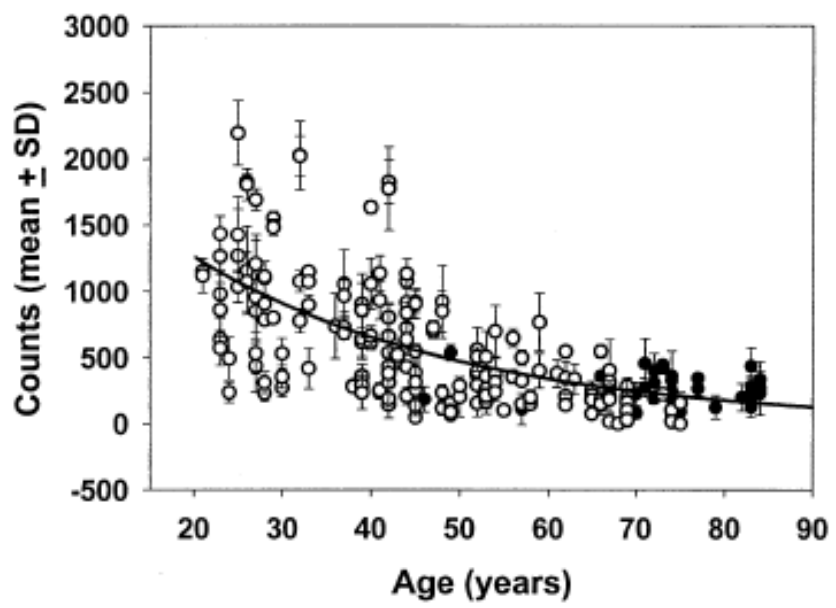




\begin{tabular}{|c|c|}
\hline Maxwellian view & Free view \\
\hline Complex optical system. & Simplified optical system. \\
\hline $\begin{array}{l}\text { Test stimuli produced by a single light } \\
\text { source, e.g. a quartz halogen lamp, an } \\
\text { advantage being that deterioration of the } \\
\text { equipment with age or voltage fluctuations } \\
\text { alter both stimuli equally and therefore } \\
\text { should not affect the results [49]. }\end{array}$ & $\begin{array}{l}\text { Test stimuli produced by light-emitting } \\
\text { diodes (LEDs) with appropriate peak } \\
\text { wavelengths. }\end{array}$ \\
\hline $\begin{array}{l}\text { Light from the lamp is rendered into the } \\
\text { appropriate monochromatic wavelengths } \\
\text { using filters. }\end{array}$ & $\begin{array}{l}\text { LEDs have the advantage of producing near } \\
\text { monochromatic light, i.e. no need for filters } \\
{[48] \text {. }}\end{array}$ \\
\hline $\begin{array}{l}\text { Test and reference lights alternated by a } \\
\text { rotating chopper [e.g. 49] or mirror [e.g. 43- } \\
\text { 46], with the radiance of the test light } \\
\text { adjustable by a 'highly linear, compensated, } \\
\text { neutral density wedge' [49]. }\end{array}$ & $\begin{array}{l}\text { Alternation of the test and reference lights is } \\
\text { achieved by square wave current pulses } \\
\text { [48]. The use of LEDs also permits the } \\
\text { possibility of adjusting the test light radiance } \\
\text { directly through voltage, thereby simplifying } \\
\text { the use of the instrument and avoiding any } \\
\text { need for rotating mirrors and neutral density } \\
\text { wedges (see Wooten et al. [46]). }\end{array}$ \\
\hline $\begin{array}{l}\text { Narrow beam light stimuli must enter the eye } \\
\text { precisely in the centre of the pupil, which } \\
\text { requires head stabilization with a dental bite } \\
\text { bar }[46,65] \text {. }\end{array}$ & $\begin{array}{l}\text { Wider beam light stimuli can enter the eye } \\
\text { through the whole pupil so head stabilization } \\
\text { with a bite bar is not necessary }[46,65] \text {. }\end{array}$ \\
\hline Not easily portable. & Portable. \\
\hline Expensive. & Relatively inexpensive. \\
\hline $\begin{array}{l}\text { Considerable training required to operate } \\
\text { correctly. }\end{array}$ & $\begin{array}{l}\text { Simpler to operate than Maxwellian systems } \\
\text { therefore less training required. }\end{array}$ \\
\hline
\end{tabular}




\begin{tabular}{|c|c|c|c|}
\hline Study/Year & $\begin{array}{l}\text { Number of } \\
\text { subjects }\end{array}$ & $\begin{array}{c}\text { Age range; eye health } \\
\text { status; experience with HFP }\end{array}$ & Reliability statistics \\
\hline $\begin{array}{l}\text { Hammond and } \\
\text { Fuld/1992 [43] }\end{array}$ & 10 & 19-42; normal; 7 novices. & $\begin{array}{l}5 \text { sessions on different days. Intersession correlation } 0.85 \\
\text { (Cronbach's } \alpha) \text {. Mean absolute difference between } 1^{\text {st }} \text { and } 2^{\text {nd }} \\
\text { session } 0.05 \pm 0.03(16 \% \text { of mean MPOD, as cited in Delori et } \\
\text { al. [56]; } Y=0.87 X+0.03, r=0.94(p<0.0005)) \text {. }\end{array}$ \\
\hline $\begin{array}{l}\text { Hammond, Fuld and } \\
\text { Curran-Celentano/1995 } \\
\text { [82] }\end{array}$ & 20 & 19-22; normal; all novices. & $\begin{array}{l}5 \text { sessions on different days. Intersession correlation } 0.68 \\
\text { (Cronbach's } \alpha) \text {. Mean absolute difference between } 1^{\text {st }} \text { and } 2^{\text {nd }} \\
\text { session } 0.10 \pm 0.10(33 \% \text { of mean MPOD, as cited in Delori et } \\
\text { al. [56]; } Y=0.56 X+0.12, r=0.73(p<0.0005)) \text {. }\end{array}$ \\
\hline $\begin{array}{l}\text { Hammond, Wooten and } \\
\text { Snodderly/1997 [44] }\end{array}$ & 32 & 21-63; normal; 29 novices. & $\begin{array}{l}2 \text { sessions on different days. Mean absolute difference } \\
\text { between sessions } 0.08 \pm 0.08 ; Y=0.92 X+0.07, r=0.91 \text { (no } \\
\text { p-value given). }\end{array}$ \\
\hline $\begin{array}{l}\text { Hammond et al./1997 } \\
\text { [67] }\end{array}$ & 13 & 30-65; normal; not reported. & $\begin{array}{l}2 \text { sessions. Mean absolute difference between sessions } 0.08 \pm \\
0.07(23 \% \text { of mean MPOD, as cited in Delori et al. [56]; } \mathrm{Y}= \\
0.83 \mathrm{X}+0.03, r=0.72 \text { (no } \mathrm{p} \text {-value given)). }\end{array}$ \\
\hline $\begin{array}{l}\text { Hammond, Wooten and } \\
\text { Snodderly/1998 [105] }\end{array}$ & $10+27$ & $\begin{array}{l}24-36 \& 60-84 ; \text { some older } \\
\text { subjects with mild RPE } \\
\text { changes and } 2 \text { with early } \\
\text { cataract; not reported. }\end{array}$ & $\begin{array}{l}2 \text { sessions. Mean absolute difference between sessions } 0.08 \pm \\
0.09 ; Y=0.90 X+0.06, r=0.91(p<0.0000001) .\end{array}$ \\
\hline Wooten & 4 & $\begin{array}{l}30 \text { subjects in full study, } 16- \\
60 ; \text { not reported; not } \\
\text { specifically reported. }\end{array}$ & $\begin{array}{l}10 \text { sessions over } 2-4 \text { weeks. Intersession correlation } 0.89 \\
\text { (Cronbach's } \alpha \text { ). }\end{array}$ \\
\hline $\begin{array}{l}\text { Hammond and Caruso- } \\
\text { Avery/2000 [11] }\end{array}$ & 8 & $\begin{array}{l}217 \text { subjects in full study, } \\
17-92 ; \text { normal; all novices. }\end{array}$ & $\begin{array}{l}10 \text { sessions over } 2-4 \text { weeks. Intersession correlation } 0.97 \\
\text { (Cronbach's } \alpha \text { ). Average range over sessions }=0.166 \text { (best to } \\
\text { worst } 0.07-0.27) \text {. Mean absolute difference between } 2 \\
\text { sessions } 0.04 \pm 0.04 \text { (18\% of mean MPOD, as cited in Delori et } \\
\text { al. [56]). }\end{array}$ \\
\hline Ciulla et al./2001 [81] & 24 & $\begin{array}{l}48-82 ; \text { pre-post cataract; all } \\
\text { novices. }\end{array}$ & $\begin{array}{l}\text { Mean absolute difference between } 1^{\text {st }}\left(\text { pre cataract op) and } 2^{\text {nd }}\right. \\
\text { (post cataract op) session } 0.085 \pm 0.08 ; Y=0.53 X+0.07, r= \\
0.58(p<0.001) \text {. Excluding } 2 \text { outliers strengthens the } \\
\text { relationship to } Y=0.69 X+0.03, r=0.75(p<0.0001) \text {. }\end{array}$ \\
\hline Mellerio et al./2002 [48] & 3 & $\begin{array}{l}124 \text { subjects in full study, } \\
18-84 ; \text { normal; not reported. }\end{array}$ & $\begin{array}{l}4 \text { sessions on successive days. Coefficients of variation = } \\
8.6 \%, 5.1 \% \text { and } 6.7 \% \text { for subjects } 1,2 \text { and } 3 \text { respectively. }\end{array}$ \\
\hline
\end{tabular}




\begin{tabular}{|c|c|c|c|}
\hline $\begin{array}{l}\text { Bernstein et al./2004 } \\
{[110]}\end{array}$ & 40 & $<60$; normal; not reported. & 3 sessions over 1 month. Mean intersession variability $10.1 \%$. \\
\hline Koh et al./2004 [91] & $7+6$ & $\begin{array}{l}\text { 58-81; } 7 \text { early ARM, } 6 \\
\text { normal; not reported. }\end{array}$ & $\begin{array}{l}2 \text { sessions } 1-4 \text { weeks apart. Mean absolute difference between } \\
\text { sessions } 0.063 \pm 0.054 \text { (normal eyes), } 0.037 \pm 0.02(\mathrm{ARM} \\
\text { eyes) and } 0.041 \pm 0.027 \text { (fellow eyes); } Y=0.95 X+0.019, r= \\
0.88 \text { (no p-value given). }\end{array}$ \\
\hline Nolan et al./2004 [107] & 100 & 22-60; normal; not reported. & $\begin{array}{l}2 \text { sessions at least } 90 \text { mins apart. Mean difference between } \\
\text { sessions }-0.01 \pm 0.08 \text {. }\end{array}$ \\
\hline $\begin{array}{l}\text { Snodderly et al./2004 } \\
{[50]}\end{array}$ & 48 & $\begin{array}{l}50-79 ; 15 \text { with self-reported } \\
\text { eye disease; all novices. }\end{array}$ & $\begin{array}{l}2 \text { sessions on different days. Test-retest correlation at } 0.5^{\circ} \mathrm{RE} \\
\text { was } r=0.90 \text { (no } \mathrm{p} \text {-value given); } \mathrm{Y}=0.94 \mathrm{X}+0.02 . \text { Mean } \\
\text { absolute test-retest difference (as a percentage of mean } \\
\text { density) }=17 \% \text {. Coefficient of repeatability }=0.19 .^{\mathrm{a}}\end{array}$ \\
\hline Tang et al./2004 [62] (i) & 6 & $\begin{array}{l}68 \text { subjects in full study, 22- } \\
23 ; \text { normal; not reported. }\end{array}$ & $\begin{array}{l}3 \text { sessions, each separated by a week. Coefficient of variation } \\
=8.0 \% \text { and coefficient of repeatability }=0.12 \text {. }\end{array}$ \\
\hline $\begin{array}{l}\text { Tang et al./2004 [62] } \\
\text { (ii) }\end{array}$ & 1 & 45; normal; not reported. & $\begin{array}{l}5 \text { sessions on consecutive days. Coefficient of variation }= \\
7.2 \% \text {. }\end{array}$ \\
\hline $\begin{array}{l}\text { Hammond and } \\
\text { Wooten/2005 [86] }\end{array}$ & 8 & 20-30; normal; novices. & $\begin{array}{l}10 \text { separate sessions over } 2-4 \text { weeks. Intersession correlation } \\
0.97 \text { (Cronbach's } \alpha \text { ). }\end{array}$ \\
\hline Lam et al./2005 [70] & 9 & $\begin{array}{l}\text { Average age } 31.2 ; \text { normal; } \\
\text { novices. }\end{array}$ & $\begin{array}{l}2 \text { sessions on different days. Test-retest correlation at } 0.5^{\circ} \text { was } \\
r=0.68 \text { (no } p \text {-value given). }\end{array}$ \\
\hline Liew et al./2005 [164] & 17 & $\begin{array}{l}150 \text { twin pairs in full study, } \\
18-50 ; \text { normal; not reported. }\end{array}$ & $\begin{array}{l}2 \text { sessions on different days. Mean difference between } \\
\text { sessions } 0.10 \pm 0.11 . \text { Mean coefficient of variation }=17.6 \%( \pm \\
16.5 \%) \text {. }\end{array}$ \\
\hline $\begin{array}{l}\text { Gallaher et al./2007 } \\
{[114]}\end{array}$ & 40 & $\begin{array}{l}69-84 ; \text { not specifically } \\
\text { reported; not reported. }\end{array}$ & $\begin{array}{l}2 \text { sessions, } 1 \text { week to } 20 \text { months apart. Mean difference } \\
\text { between sessions }-0.01 \pm 0.16 \text {. Test-retest correlation was } r= \\
0.734 \text { (no p-value given). Mean coefficient of variation }=18.4 \% \text {. } \\
\text { Intraclass correlation }=0.96 \text {. }\end{array}$ \\
\hline Hogg et al./2007 [176] & 11 & 21-50; normal; novices. & $\begin{array}{l}4 \text { sessions over } 2 \text { weeks. Mean coefficient of variation between } \\
\text { sessions }=11.5 \% \text { at } 0.5^{\circ} \text {. }\end{array}$ \\
\hline $\begin{array}{l}\text { Johnson et al./2008 } \\
\text { [122] }\end{array}$ & 49 & 60-80; normal; not reported. & $\begin{array}{l}2 \text { sessions 1-4 days apart. Test-retest correlation was } r=0.90 \\
\text { (no } p \text {-value given). }\end{array}$ \\
\hline Kirby et al./2009 [64] & 16 & $\begin{array}{l}\text { Not reported; normal; } \\
\text { experienced. }\end{array}$ & $\begin{array}{l}3 \text { sessions on different days. Intraclass correlations 'in the } \\
\text { range' } 0.93-0.96 \text { at } 0.25^{\circ}, 0.5^{\circ} \text { and } 1^{\circ} \text { retinal eccentricity. }\end{array}$ \\
\hline $\begin{array}{l}\text { Van der Veen et } \\
\text { al./2009 [53] }\end{array}$ & 11 & $\begin{array}{l}26 \text { subjects in full study, 22- } \\
64 \text {; normal; not reported. }\end{array}$ & $\begin{array}{l}2 \text { sessions on different days. Mean difference between } \\
\text { sessions } 0.0195 \pm 0.047 ; Y=1.2 X-0.05, r=0.97(p<0.001) \text {. }\end{array}$ \\
\hline
\end{tabular}




\begin{tabular}{|l|c|l|l|}
\hline & & & $\begin{array}{l}\text { Mean test-retest difference (as a percentage of mean density) } \\
=11.7 \% .\end{array}$ \\
\hline Bartlett et al./2010 [119] & 38 & $19-46 ;$ normal; novices. & $\begin{array}{l}2 \text { sessions 1 week apart. Test-retest coefficients of } \\
\text { repeatability were } 0.45 \text { (operator } 1 \text { ) and } 0.58 \text { (operator 2). }\end{array}$ \\
\hline Hagen et al./2010 [120] & 24 & $38.1 \pm 10.6 ;$ normal; novices. & $\begin{array}{l}3 \text { sessions over } 11 \pm 6.9 \text { days. Coefficients of variation were } \\
36.1 \% \text { and } 23 \% \text { for right and left eyes, respectively. }\end{array}$ \\
\hline
\end{tabular}




\begin{tabular}{|c|c|c|c|}
\hline Study/Year & $\begin{array}{l}\text { Number of } \\
\text { subjects }\end{array}$ & $\begin{array}{l}\text { Age range; eye health status; } \\
\text { inter or intra session reliability }\end{array}$ & Reliability statistics \\
\hline $\begin{array}{l}\text { Berendschot et al./2000 } \\
\text { [136] }\end{array}$ & 8 & 18-50; normal; intra-session. & $\begin{array}{l}2 \text { measurements. Within subjects variation and coefficients of } \\
\text { repeatability were } 10 \% \text { and } 0.17 \text { with a SLO, and } 17 \% \text { and } 0.27 \text { with } \\
\text { a purpose-built reflectometer. }\end{array}$ \\
\hline Delori et al./2001 [56] (i) & 9 & 21-72; normal; both. & $\begin{array}{l}2 \text { sessions, } 4 \text { on different days \& } 5 \text { on the same day. Mean absolute } \\
\text { difference between } 1^{\text {st }} \text { and } 2^{\text {nd }} \text { session } 0.039 \pm 0.029 \text {. Mean absolute } \\
\text { test-retest difference (as a percentage of mean density) }=22 \% \text {. }\end{array}$ \\
\hline Delori et al./2001 [56] (ii) & 22 & 22-78; normal; inter-session. & $\begin{array}{l}2 \text { sessions, } 10-24 \text { months apart. Mean absolute difference between } \\
\text { sessions } 0.042 \pm 0.042 \text {. Mean absolute test-retest difference }=19 \% \text {. }\end{array}$ \\
\hline $\begin{array}{l}\text { Berendschot et al./2002 } \\
\text { [144] }\end{array}$ & 17 & $\begin{array}{l}\text { Average age } 67.5 ; 6 \text { ARM, } 11 \\
\text { normal; intra-session. }\end{array}$ & $\begin{array}{l}2 \text { sets of measurements. Coefficient of repeatability } 0.11 \text {. Mean } \\
\text { relative difference between measurements }=10 \% \text {. }\end{array}$ \\
\hline Bour et al./2002 [137] & ‘6 eyes’ & $\begin{array}{l}23 \text { subjects in full study, 6-20; } \\
\text { normal; intra-session. }\end{array}$ & $\begin{array}{l}2 \text { sets of measurements with a fundus camera. Correlation } \\
\text { coefficient between measures was } r=0.77(p<0.05) \text {. }\end{array}$ \\
\hline $\begin{array}{l}\text { Wüstemeyer et al./2002 } \\
\text { [98] }\end{array}$ & 10 & 16-43; normal; intra-session. & $\begin{array}{l}2 \text { sessions with a SLO, no more than } 30 \text { mins apart. Mean within } \\
\text { subjects coefficient of variation }=6.2 \% \text {. As a percentage of mean } \\
\text { density, as used by Delori et al. [56], mean test-retest difference = } \\
3.1 \% \text {. }\end{array}$ \\
\hline Zagers et al./2002 [146] & 21 & $\begin{array}{l}18-27(n=15) \& 40-74(n=6) \\
\text { normal; intra-session. }\end{array}$ & $\begin{array}{l}25 \text { measurements all in the same sitting with the FRA. Coefficient of } \\
\text { repeatability } 0.084 \text {. }\end{array}$ \\
\hline $\begin{array}{l}\text { Berendschot and van } \\
\text { Norren/2005 [141] }\end{array}$ & 53 & 19-76; normal; intra-session. & $\begin{array}{l}5 \text { measurements (same sitting). Mean within subjects variation and } \\
\text { coefficients of repeatability were } 5.5 \% \text { and } 0.078 \text { with the FRA } 1 \text {, and } \\
7.0 \% \text { and } 0.09 \text { with the FRA } 2 \text {. }\end{array}$ \\
\hline $\begin{array}{l}\text { van de Kraats et al./2006 } \\
\text { [58] }\end{array}$ & 10 & $\begin{array}{l}20 \text { subjects in full study, 18-79; } \\
\text { normal; both. }\end{array}$ & $\begin{array}{l}2 \text { sessions on different days with the MPR. Test-retest correlation } \\
\text { was } r=0.94(p<0.001) .5 \text { spectra measured in each test condition } \\
\text { (intra-session), gave a mean within subjects variation of 'typically' } \\
7 \% \text {. }\end{array}$ \\
\hline Bone et al./2007 [59] & 22 & 18-24; normal; inter-session. & $\begin{array}{l}6-8 \text { sessions at } \sim 2 \text { weekly intervals with a fundus camera. Standard } \\
\text { deviation of the set of measurements was 'typically' } \leq 0.05 \text {. }\end{array}$ \\
\hline
\end{tabular}




\begin{tabular}{|c|c|c|c|}
\hline Study/Year & $\begin{array}{l}\text { Number of } \\
\text { subjects }\end{array}$ & $\begin{array}{l}\text { Age range; eye health status; } \\
\text { inter or intra session reliability }\end{array}$ & Reliability statistics \\
\hline Delori et al./2001 [56] (i) & 9 & 21-72; normal; both. & $\begin{array}{l}\text { AF comparison method: } 2 \text { sessions, } 4 \text { on different days } \& 5 \text { on the } \\
\text { same day. Mean absolute difference between } 1^{\text {st }} \text { and } 2^{\text {nd }} \text { session } \\
0.042 \pm 0.019 . \text { Mean absolute test-retest difference (as a percentage } \\
\text { of mean density) }=9.0 \% \text {. }\end{array}$ \\
\hline Delori et al./2001 [56] (ii) & 22 & 22-78; normal; inter-session. & $\begin{array}{l}\text { AF comparison method: } 2 \text { sessions, } 10-24 \text { months apart. Mean } \\
\text { absolute difference between sessions } 0.053 \pm 0.048 \text {. Mean absolute } \\
\text { test-retest difference }=11 \% \text {. }\end{array}$ \\
\hline Delori/2004 [159] & 38 & 24-77; normal; intra-session. & AF imaging: Test-retest reproducibility $0.03 \pm 0.03$. \\
\hline $\begin{array}{l}\text { Berendschot and van } \\
\text { Norren/2005 [141] }\end{array}$ & 53 & 19-76; normal; intra-session. & $\begin{array}{l}\text { AF imaging: } 10 \text { single AF images at } 488 \mathrm{~nm} \text { and } 514 \mathrm{~nm} \text { (without } \\
\text { averaging). Mean within subjects variation }=17 \% \text { and coefficient of } \\
\text { repeatability }=0.13 \text {. }\end{array}$ \\
\hline Liew et al./2005 [164] & 8 & $\begin{array}{l}150 \text { twin pairs in full study, } 18- \\
50 \text {; normal; inter-session. }\end{array}$ & $\begin{array}{l}\text { AF imaging: } 2 \text { sessions less than } 1 \text { month apart. Mean difference } \\
\text { between sessions } 0.02 \pm 0.02 \text { (range } 0-0.05) \text {. Mean coefficient of } \\
\text { variation }=3.3 \%( \pm 2.1 \%) \text {. }\end{array}$ \\
\hline Delori et al./2006 [117] & 37 & 20-70; normal; intra-session. & $\begin{array}{l}\text { AF imaging: Short break between first and second measurement. } \\
\text { Mean absolute test-retest difference (as a percentage of mean } \\
\text { density) }=6.4 \% \text { (range } 0-34 \% \text { ) for peak }\left(0^{\circ}\right) \text { MPOD and } 6.4 \% \text { (range } \\
0-36 \% \text { ) for MPOD averaged over a } 1^{\circ} \text { foveal-centred area. }\end{array}$ \\
\hline $\begin{array}{l}\text { Sharifzadeh et al./2006 } \\
\text { [153] }\end{array}$ & 1 & $\begin{array}{l}\text { Age unreported; normal; inter- } \\
\text { session. }\end{array}$ & $\begin{array}{l}\text { AF imaging: } 8 \text { measurements over } 4 \text { weeks. Standard deviation of } \\
\text { MPOD } 2.4 \% \text {. }\end{array}$ \\
\hline $\begin{array}{l}\text { Trieschmann et al./2006 } \\
\text { [162] }\end{array}$ & 20 & $\begin{array}{l}120 \text { subjects in full study, } 20- \\
86 ; 15 \text { normal, } 5 \text { AMD; intra- } \\
\text { session. }\end{array}$ & $\begin{array}{l}\text { AF imaging: } 5 \text { repeats. } 1-\lambda \text { method at } 0.5^{\circ}: \text { median coefficient of } \\
\text { variation }=3.6 \% \text { and reliability ratio }=0.97 .2-\lambda \text { method at } 0.5^{\circ}: \\
\text { median coefficient of variation }=6.8 \% \text { and reliability ratio }=0.94 \text {. }\end{array}$ \\
\hline
\end{tabular}




\begin{tabular}{|c|c|c|c|}
\hline Study/Year & $\begin{array}{l}\text { Number of } \\
\text { subjects }\end{array}$ & $\begin{array}{l}\text { Age range; eye health status; } \\
\text { inter or intra session reliability }\end{array}$ & Reliability statistics \\
\hline $\begin{array}{l}\text { Bernstein et al./2002 } \\
{[170]}\end{array}$ & 2 & 26 and 37 ; normal; both. & $\begin{array}{l}5 \text { sessions over } 2 \text { weeks. Variability within and between sessions } \\
\text { was 'generally less than } \pm 10 \% \text { '. }\end{array}$ \\
\hline $\begin{array}{l}\text { Gellermann et al./2002 } \\
\text { [171] }\end{array}$ & 2 & 26 and 37 ; normal; both. & $\begin{array}{l}5 \text { sessions over } 2 \text { weeks. Standard deviation within sessions ( } 5 \\
\text { readings) was typically less than } 15 \% \text {. Reproducibility between } \\
\text { sessions was 'high'. }\end{array}$ \\
\hline $\begin{array}{l}\text { Bernstein et al./2004 } \\
{[110]}\end{array}$ & 40 & $<60$; normal; inter-session. & 3 sessions over 1 month. Mean intersession variability $6.4 \%$. \\
\hline Ermakov et al./2004 [174] & 2 & Age unreported; normal; both. & $\begin{array}{l}\text { Subject } 1: 6 \text { measurements with 3-4 minute intervals. Relative } \\
\text { standard deviation }=5.3 \% \text {. } \\
\text { Subject } 2: 5 \text { sessions over } 2 \text { weeks. Relative standard deviation = } \\
5.1 \% \text {. }\end{array}$ \\
\hline Neelam et al./2005 [57] & 20 & $\begin{array}{l}120 \text { subjects in full study, } 20- \\
60 ; \text { normal; both. }\end{array}$ & $\begin{array}{l}2 \text { sessions less than } 2 \text { weeks apart. Within-session mean coefficient } \\
\text { of variation (using three highest readings) }=12.61 \%( \pm 9.46 \%) \text { at } \\
\text { session } 1 \text { and } 8.42 \%( \pm 7.12 \%) \text { at session } 2 . \text { Bland-Altman plots for } \\
\text { inter-session reproducibility showed that } 95 \% \text { of MPOD readings } \\
\text { were within the } 95 \% \text { limits of agreement. }\end{array}$ \\
\hline Hogg et al./2007 [176] & 11 & 21-50; normal; inter-session. & $\begin{array}{l}4 \text { sessions over } 2 \text { weeks. Mean coefficient of variation between } \\
\text { sessions }=13.5 \% \text {. }\end{array}$ \\
\hline Obana et al./2008 [177] & 197 & $\begin{array}{l}20-80 \& 50-85 ; 100 \text { normal, } 97 \\
\text { ARM/AMD; intra-session. }\end{array}$ & $\begin{array}{l}\text { Coefficient of variation of } 5 \text { readings was } 1.0 \%-69.4 \% \text { in normal } \\
\text { subjects and } 0.9 \%-145.4 \% \text { in ARM subjects. }\end{array}$ \\
\hline
\end{tabular}


Click here to download Data not for Review: Permissions.docx (1)

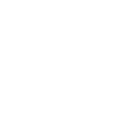

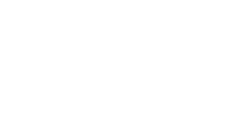


Permission evidence for new fig. 14
Click here to download Data not for Review: Permission for new fig. 14.pdf

Permission evidence for new fig. 14
Click here to download Data not for Review: Permission for new fig. 14.pdf

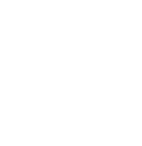

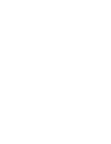

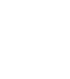

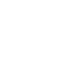

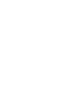

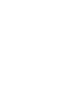

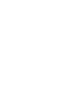
.

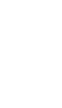

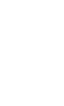

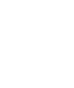

